\title{
More asymptotic safety guaranteed
}

\author{
Andrew D. Bond ${ }^{*}$ and Daniel F. Litim ${ }^{\dagger}$ \\ Department of Physics and Astronomy, U Sussex, Brighton BN1 9QH, United Kingdom
}

(Received 2 February 2018; published 10 April 2018)

\begin{abstract}
We study interacting fixed points and phase diagrams of simple and semisimple quantum field theories in four dimensions involving non-Abelian gauge fields, fermions and scalars in the Veneziano limit. Particular emphasis is put on new phenomena which arise due to the semisimple nature of the theory. Using matter field multiplicities as free parameters, we find a large variety of interacting conformal fixed points with stable vacua and crossovers inbetween. Highlights include semisimple gauge theories with exact asymptotic safety, theories with one or several interacting fixed points in the IR, theories where one of the gauge sectors is both UV free and IR free, and theories with weakly interacting fixed points in the UV and the IR limits. The phase diagrams for various simple and semisimple settings are also given. Further aspects such as perturbativity beyond the Veneziano limit, conformal windows, and implications for model building are discussed.
\end{abstract}

DOI: 10.1103/PhysRevD.97.085008

\section{INTRODUCTION}

Asymptotic freedom is a key feature of non-Abelian gauge theories $[1,2]$. It predicts that interactions weaken with growing energy due to quantum effects, thereby reaching a free ultraviolet (UV) fixed point under the renormalization group. Asymptotic safety, on the other hand, stipulates that running couplings may very well asymptote into an interacting UV fixed point at highest energies $[3,4]$. The most striking difference between asymptotically free and asymptotically safe theories relates to residual interactions in the UV. Canonical power counting is modified, whence establishing asymptotic safety in a reliable manner becomes a challenging task [5].

Rigorous results for asymptotic safety at weak coupling have been known since long for models including either scalars, fermions, gauge fields or gravitons, and away from their respective critical dimensionality $[4,6-16]$. In these toy models asymptotic safety arises through the cancellation of tree level and leading order quantum terms. Progress has also been made to substantiate the asymptotic safety conjecture beyond weak coupling [5]. This is of particular relevance for quantum gravity where good evidence has arisen in a variety of different settings [17-31].

\footnotetext{
a.bond@sussex.ac.uk

†.litim@sussex.ac.uk
}

Published by the American Physical Society under the terms of the Creative Commons Attribution 4.0 International license. Further distribution of this work must maintain attribution to the author(s) and the published article's title, journal citation, and DOI. Funded by SCOAP ${ }^{3}$.
An important new development in the understanding of asymptotic safety has been initiated in [32] where it was shown that certain four-dimensional quantum field theories involving $S U(N)$ gluons, quarks, and scalars can develop weakly coupled UV fixed points. Results have been extended beyond classically marginal interactions [33]. Structural insights into the renormalization of general gauge theories have led to necessary and sufficient conditions for asymptotic safety, alongside strict no go theorems [34,35]. Asymptotic safety invariably arises as a quantum critical phenomenon through cancellations at loop level for which all three types of elementary degrees of freedom-scalars, fermions, and gauge fields-are required. Findings have also been extended to cover supersymmetry [36] and UV conformal windows [37]. Throughout, it is found that suitable Yukawa interactions are pivotal $[34,35]$.

In this paper, we are interested in fixed points of semisimple gauge theories. Our primary motivation is the semisimple nature of the standard model, and the prospect for asymptotically safe extensions thereof [38]. We are particularly interested in semisimple theories where interacting fixed points and asymptotic safety can be established rigorously [34]. More generally, we also wish to understand how low- and high-energy fixed points are generated dynamically, what their features are, and whether novel phenomena arise owing to the semisimple nature of the underlying gauge symmetry. Understanding the stability of a Higgs-like ground state at interacting fixed points is also of interest in view of the "near-criticality" of the standard model vacuum $[39,40]$.

We investigate these questions for quantum field theories with $S U\left(N_{C}\right) \times S U\left(N_{c}\right)$ local gauge symmetry coupled to 
massless fermionic and singlet scalar matter. Our models also have a global $U\left(N_{\mathrm{F}}\right)_{L} \times U\left(N_{\mathrm{F}}\right)_{R} \times U\left(N_{\mathrm{f}}\right)_{L} \times U\left(N_{\mathrm{f}}\right)_{R}$ flavor symmetry, and are characterized by up to nine independent couplings. Matter field multiplicities serve as free parameters. We obtain rigorous results from the leading orders in perturbation theory by adopting a Veneziano limit. We then provide a comprehensive classification of quantum field theories according to their UV and IR limits, their fixed points, and eigenvalue spectra. Amongst these, we find semisimple gauge theories with exact asymptotic safety in the UV. We also find a large variety of theories with crossover- and low-energy fixed points. Further novelties include theories with inequivalent yet fully attractive IR conformal fixed points, theories with weakly interacting fixed points in both the UV and the IR, and massless theories with a nontrivial gauge sector which is UV free and IR free. We illustrate our results by providing general phase diagrams for simple and semisimple gauge theories with and without Yukawa interactions.

The paper is organized as follows. General aspects of weakly interacting fixed points in $4 d$ gauge theories are laid out in Sec. II, together with first results and expressions for universal exponents. In Sec. III we introduce concrete families of semisimple gauge theories coupled to elementary singlet "mesons" and suitably charged massless fermions. Perturbative RG equations for all gauge, Yukawa and scalar couplings and masses in a Veneziano limit are provided to the leading non-trivial orders in perturbation theory. Section IV presents our results for all interacting perturbative fixed points and their universal scaling exponents. Particular attention is paid to new effects which arise due to the semisimple nature of the models. Section V provides the corresponding fixed points in the scalar sector. It also establishes stability of the quantum vacuum whenever a physical fixed point arises in the gauge sector. Using field multiplicities as free parameters, Sec. VI provides a complete classification of distinct models with asymptotic freedom or asymptotic safety in the UV, or without UV completions, together with their scaling in the deep IR. In Sec. VII, the generic phase diagrams for simple and semisimple gauge theories with and without Yukawas are discussed. The phase diagrams, UV-IR transitions, and aspects of IR conformality are analysed in more depth for sample theories with asymptotic freedom and asymptotic safety. Further reaching topics such as exact perturbativity, extensions beyond the Veneziano limit, and conformal windows are discussed in Sec. VIII. Section IX closes with a brief summary.

\section{FIXED POINTS OF GAUGE THEORIES}

In this section, we discuss general aspects of interacting fixed points in semisimple gauge theories which are weakly coupled to matter, with or without Yukawa interactions, following $[34,35]$. We also introduce some notation and conventions.

\section{A. Fixed points in perturbation theory}

We are interested in the renormalization of general gauge theories coupled to matter fields, with or without Yukawa couplings. The running of the gauge couplings $\alpha_{i}=g_{i}^{2} /(4 \pi)^{2}$ with the renormalization group scale $\mu$ is determined by the beta functions of the theory. Expanding them perturbatively up to two loop we have

$$
\mu \partial_{\mu} \alpha_{i} \equiv \beta_{i}=\alpha_{i}^{2}\left(-B_{i}+C_{i j} \alpha_{j}-2 Y_{4, i}\right)+O\left(\alpha^{4}\right),
$$

where a sum over gauge group factors $j$ is implied. The one- and two-loop gauge contributions $B_{i}$ and $C_{i j}$ and the two-loop Yukawa contributions $Y_{4, i}$ are known for general gauge theories, see [34,41-44] for explicit expressions. While $B_{i}$ and $C_{i i}$ may take either sign, depending on the matter content, the Yukawa contribution $Y_{4, i}$ and the offdiagonal gauge contributions $C_{i j}(i \neq j)$ are strictly positive in any quantum field theory. Scalar couplings do not play any role at this order in perturbation theory. The effect of Yukawa couplings is incorporated by projecting the gauge beta functions (1) onto the Yukawa nullclines $\left(\beta_{Y}=0\right)$, leading to explicit expressions for $Y_{4, i}$ in terms of the gauge couplings $g_{j}$. Moreover, for many theories the Yukawa contribution along nullclines can be written as $Y_{4, i}=D_{i j} \alpha_{j}$ with $D_{i j} \geq 0$ [34]. We can then go one step further and express the net effect of Yukawa couplings as a shift of the two loop gauge contribution, $C_{i j} \rightarrow C_{i j}^{\prime}=$ $C_{i j}-2 D_{i j} \leq C_{i j}$. Notice that the shift will always be by some negative amount provided at least one of the Yukawa couplings is nonvanishing. It leads to the reduced gauge beta functions

$$
\beta_{i}=\alpha_{i}^{2}\left(-B_{i}+C_{i j}^{\prime} \alpha_{j}\right)+O\left(\alpha^{4}\right) .
$$

Fixed points solutions of (2) are either free or interacting and $\alpha^{*}=0$ for some or all gauge factors is always a selfconsistent solution. Consequently, interacting fixed points are solutions to

$$
B_{i}=C_{i j}^{\prime} \alpha_{j}^{*}, \quad \text { subject to } \alpha_{i}^{*}>0,
$$

where only those rows and columns are retained where gauge couplings are interacting.

Next we discuss the role of Yukawa couplings for the fixed point structure. In the absence of Yukawa couplings, the two-loop coefficients remain unshifted $C_{i j}{ }^{\prime}=C_{i j}$. An immediate consequence of this is that any interacting fixed point must necessarily be IR. The reason is as follows: for an interacting fixed point to be UV, asymptotic freedom cannot be maintained for all gauge factors, meaning that some $B_{i}<0$. However, as has been established in [34], $B_{i} \leq 0$ necessarily entails $C_{i j} \geq 0$ in any $4 d$ quantum gauge theory. If the left-hand side of (3) is negative, if only for a single row, positivity of $C_{i j}$ requires that some $\alpha_{j}^{*}$ must take 
negative values for a fixed point solution to arise. This, however, is unphysical [45] and we are left with $B_{i}>0$ for each $i$, implying that asymptotic freedom remains intact in all gauge sectors. Besides the Gaussian, the theory may have weakly interacting infrared Banks-Zaks fixed points in each gauge sector, as well as products thereof, which arise as solutions to (3) with the unshifted coefficients.

In the presence of Yukawa couplings, the coefficients $C_{i j}{ }^{\prime}$ can in general take either sign. This has far reaching implications. First, the theory can additionally display gauge-Yukawa fixed points where both the gauge and the Yukawa couplings take interacting values. Most importantly, solutions to (3) are then no longer limited to theories with asymptotic freedom. Instead, interacting fixed points can be infrared, ultraviolet, or of the crossover type. In general we may expect gauge-Yukawa fixed points for each independent Yukawa nullcline. In summary, perturbative fixed points are either (i) free and given by the Gaussian, or (ii) free in the Yukawa but interacting in the gauge sector (Banks-Zaks fixed points), or (iii) simultaneously interacting in the gauge and the Yukawa sector (gauge-Yukawa fixed points), or (iv) combinations and products of (i), (ii), and (iii). Banks-Zaks fixed points are always IR, while the Gaussian and gauge-Yukawa fixed points can be either UV or IR. Depending on the details of the theory and its Yukawa structure, either the Gaussian or one of the interacting gauge-Yukawa fixed points will arise as the "ultimate" UV fixed point of the theory and may serve to define the theory fundamentally [35].

The effect of scalar quartic self-couplings on the fixed point is strictly subleading in terms of the values of the fixed points, as they do not affect the running of gauge couplings at this order of perturbation theory. However, as to have a true fixed point we must acquire one in all couplings, they provide additional constraints on the physicality of candidate gauge-Yukawa fixed points, as we additionally require that the quartic couplings take fixed points which are both real-valued, and lead to a bounded potential which leads to a stable vacuum state.

\section{B. Gauge couplings}

Let us now consider a semisimple gauge-Yukawa theory with non-Abelian gauge fields under the semisimple gauge group $\mathcal{G}_{1} \otimes \mathcal{G}_{2}$ coupled to fermions and scalars. We have two non-Abelian gauge couplings $\alpha_{1}$ and $\alpha_{2}$, which are related to the fundamental gauge couplings via $\alpha_{i}=g_{i}^{2} /(4 \pi)^{2}$. The running of gauge couplings within perturbation theory is given by

$$
\begin{aligned}
& \beta_{1}=-B_{1} \alpha_{1}^{2}+C_{1} \alpha_{1}^{3}+G_{1} \alpha_{1}^{2} \alpha_{2}, \\
& \beta_{2}=-B_{2} \alpha_{2}^{2}+C_{2} \alpha_{2}^{3}+G_{2} \alpha_{2}^{2} \alpha_{1} .
\end{aligned}
$$

Here, $B_{i}$ are the well known one-loop coefficients. In theories without Yukawa interactions, or where Yukawa
TABLE I. Conventions to denote the basic fixed points (Gaussian, Banks-Zaks, or gauge-Yukawa) of simple gauge theories weakly coupled to matter.

\begin{tabular}{lccc}
\hline \hline Fixed point & & $\alpha_{\text {gauge }}$ & $\alpha_{\text {Yukawa }}$ \\
\hline Gauss & $\mathrm{G}$ & $=0$ & $=0$ \\
Banks-Zaks & $\mathrm{BZ}$ & $\neq 0$ & $=0$ \\
Gauge-Yukawa & $\mathrm{GY}$ & $\neq 0$ & $\neq 0$ \\
\hline \hline
\end{tabular}

interactions take Gaussian values, the numbers $C_{i}$ and $G_{i}$ are the two-loop coefficients which arise owing to the gauge loops and owing to the mixing between gauge groups, meaning $C_{i} \equiv C_{i i}$ (no sum), and $G_{1} \equiv C_{12}, G_{2} \equiv C_{12}$, see (1). In this case, we also have that $C_{i}, G_{i} \geq 0$ as soon as $B_{i}<0 .{ }^{1}$ For theories where Yukawa couplings take interacting fixed points the numbers $C_{i}$ and $G_{i}$ receive corrections due to the Yukawas, $C_{i} \equiv C_{i i}^{\prime}$ (no sum), and $G_{1} \equiv C_{12}^{\prime}, G_{2} \equiv C_{12}^{\prime}$, see (2). Most notably, strict positivity of $C_{i}$ and $G_{i}$ is then no longer guaranteed [34].

In either case, the fixed points of the combined system are determined by the vanishing of (4). For a general semisimple gauge theory with two gauge factors, one finds four different types of fixed points. The Gaussian fixed point

$$
\left(\alpha_{1}^{*}, \alpha_{2}^{*}\right)=(0,0)
$$

always exists (see Table I for our conventions). It is the UV fixed point of the theory as long as the one-loop coefficients obey $B_{i}>0$. The theory may also develop partially interacting fixed points,

$$
\begin{aligned}
& \left(\alpha_{1}^{*}, \alpha_{2}^{*}\right)=\left(0, \frac{B_{2}}{C_{2}}\right), \\
& \left(\alpha_{1}^{*}, \alpha_{2}^{*}\right)=\left(\frac{B_{1}}{C_{1}}, 0\right) .
\end{aligned}
$$

Here, one of the gauge coupling is taking Gaussian values whereas the other one is interacting. The interacting fixed point is of the Banks-Zaks type [46,47], provided Yukawa interactions are absent. This then also implies that the gauge coupling is asymptotically free. Alternatively, the interacting fixed point can be of the gauge-Yukawa type, provided that Yukawa couplings take an interacting fixed point themselves. In this case, and depending on the details of the Yukawa sector, the fixed point can be either IR or UV. Finally, we also observe fully interacting fixed points

$$
\left(\alpha_{1}^{*}, \alpha_{2}^{*}\right)=\left(\frac{C_{2} B_{1}-B_{2} G_{1}}{C_{1} C_{2}-G_{1} G_{2}}, \frac{C_{1} B_{2}-B_{1} G_{2}}{C_{1} C_{2}-G_{1} G_{2}}\right) .
$$

\footnotetext{
${ }^{1}$ General formal expressions of loop coefficients in the conventions used here are given in [34].
} 
As such, fully interacting fixed points (8) can be either UV or IR, depending on the specific field content of the theory. In all cases we will additionally require that the couplings obey

$$
\alpha_{1} \geq 0, \quad \alpha_{2} \geq 0
$$

to ensure they reside in the physical regime of the theory [45].

\section{Yukawa couplings}

In order to proceed, we must specify the Yukawa sector. We assume three types of nontrivially charged fermions with charges under $\mathcal{G}_{1}$ and $\mathcal{G}_{2}$. Some or all of the fermions which are only charged under $\mathcal{G}_{1}\left(\mathcal{G}_{2}\right)$ also couple to scalar fields via Yukawa couplings $\alpha_{Y}\left(\alpha_{y}\right)$, respectively. The scalars may or may not be charged under the gauge symmetries. They will have quartic self couplings which play no primary role for the fixed point analysis at weak coupling [34]. Within perturbation theory, the beta functions for the gauge and Yukawa couplings are of the form

$$
\begin{aligned}
& \beta_{1}=-B_{1} \alpha_{1}^{2}+C_{1} \alpha_{1}^{3}-D_{1} \alpha_{1}^{2} \alpha_{Y}+G_{1} \alpha_{1}^{2} \alpha_{2}, \\
& \beta_{Y}=E_{1} \alpha_{Y}^{2}-F_{1} \alpha_{Y} \alpha_{1}, \\
& \beta_{2}=-B_{2} \alpha_{2}^{2}+C_{2} \alpha_{2}^{3}-D_{2} \alpha_{2}^{2} \alpha_{y}+G_{2} \alpha_{2}^{2} \alpha_{1}, \\
& \beta_{y}=E_{2} \alpha_{y}^{2}-F_{2} \alpha_{y} \alpha_{2} .
\end{aligned}
$$

The renormalization group (RG) flow is given up to two-loop in the gauge couplings, and up to one-loop in the Yukawa couplings. We refer to this as the next-to-leading-order (NLO) approximation, see Table II for the terminology.

We are interested in the fixed points of the theory, defined implicitly via the vanishing of the beta functions for all couplings. The Yukawa couplings can display either a Gaussian or an interacting fixed point

$$
\begin{array}{ll}
\alpha_{Y}^{*}=0, & \alpha_{Y}^{*}=\frac{F_{1}}{E_{1}} \alpha_{1}^{*}, \\
\alpha_{y}^{*}=0, & \alpha_{y}^{*}=\frac{F_{2}}{E_{2}} \alpha_{2}^{*} .
\end{array}
$$

Depending on whether none, one, or both of the Yukawa couplings take an interacting fixed point, the system (10)

TABLE II. Relation between approximation level and the loop order up to which couplings are retained in perturbation theory, following the terminology of $[32,48]$.

\begin{tabular}{lcccc}
\hline \hline Coupling & \multicolumn{4}{c}{ Order in perturbation theory } \\
\hline$\beta_{\text {gauge }}$ & 1 & 2 & 2 & $n+1$ \\
$\beta_{\text {Yukawa }}$ & 0 & 1 & 1 & $n$ \\
$\beta_{\text {scalar }}$ & 0 & 0 & 1 & $n$ \\
Approximation & $\mathrm{LO}$ & $\mathrm{NLO}$ & $\mathrm{NLO}^{\prime}$ & $n \mathrm{NLO}^{\prime}$ \\
\hline \hline
\end{tabular}

reduces to (4) whereby the two-loop coefficients $C_{i}$ of the gauge beta functions are shifted according to

$$
\begin{aligned}
& \alpha_{Y}^{*} \neq 0: C_{1} \rightarrow C_{1}^{\prime}=C_{1}-D_{1} \frac{F_{1}}{E_{1}} \leq C_{1}, \\
& \alpha_{y}^{*} \neq 0: C_{2} \rightarrow C_{2}^{\prime}=C_{2}-D_{2} \frac{F_{2}}{E_{2}} \leq C_{2} .
\end{aligned}
$$

Notice also that in this model the values for the mixing terms $G_{i}$ do not depend on whether the corresponding Yukawa couplings vanish, or not, due to the fact that no fermions charged under both groups are involved in Yukawa interactions. Owing to the fixed point structure of the Yukawa sector (11), the formal fixed points (5), (6), (7), and (8) have the multiplicity 1,2,2, and 4, respectively. In total, we end up with nine qualitatively different fixed points $\mathrm{FP}_{1}-\mathrm{FP}_{9}$, summarized in Table III: $\mathrm{FP}_{1}$ denotes the unique Gaussian fixed point. $\mathrm{FP}_{2}$ and $\mathrm{FP}_{3}$ correspond to a Banks-Zaks fixed point in one of the gauge couplings, and a Gaussian in the other. They can therefore be interpreted effectively as a "product" of a Banks-Zaks with a Gaussian fixed point. Similarly, at $\mathrm{FP}_{4}$ and $\mathrm{FP}_{5}$, one of the Yukawa couplings remains interacting, and they can therefore effectively be viewed as the product of a gauge-Yukawa (GY) type fixed point in one gauge coupling with a Gaussian fixed point in the other. The remaining fixed points $\mathrm{FP}_{6}-\mathrm{FP}_{9}$ are interacting in both gauge couplings. These fixed points are the only ones which are sensitive to the two-loop mixing coefficients $G_{1}$ and $G_{2}$. At $\mathrm{FP}_{6}$, both Yukawa couplings vanish meaning that it is effectively a

\begin{tabular}{|c|c|c|c|c|c|}
\hline \multirow{2}{*}{$\begin{array}{l}\text { Fixed } \\
\text { point }\end{array}$} & \multicolumn{2}{|c|}{ Gauge couplings } & \multicolumn{2}{|c|}{ Yukawa couplings } & \multirow{2}{*}{$\begin{array}{l}\text { Fixed point } \\
\text { type }\end{array}$} \\
\hline & $\alpha_{1}^{*}$ & $\alpha_{2}^{*}$ & $\alpha_{Y}^{*}$ & $\alpha_{y}^{*}$ & \\
\hline $\mathbf{F P}_{1}$ & 0 & 0 & 0 & 0 & $\mathbf{G} \cdot \mathbf{G}$ \\
\hline $\mathbf{F P}_{2}$ & $\frac{B_{1}}{C_{1}}$ & 0 & 0 & 0 & $\mathbf{B Z} \cdot \mathbf{G}$ \\
\hline $\mathbf{F P}_{\mathbf{3}}$ & 0 & $\frac{B_{2}}{C_{2}}$ & 0 & 0 & $\mathbf{G} \cdot \mathbf{B Z}$ \\
\hline $\mathbf{F P}_{4}$ & $\frac{B_{1}}{C_{1}}$ & 0 & $\frac{F_{1}}{E_{1}} \alpha_{1}$ & 0 & $\mathbf{G Y} \cdot \mathbf{G}$ \\
\hline $\mathbf{F P}_{5}$ & 0 & $\frac{B_{2}}{C_{2}^{\prime}}$ & 0 & $\frac{F_{2}}{E_{2}} \alpha_{2}$ & $\mathbf{G} \cdot \mathbf{G Y}$ \\
\hline $\mathbf{F P}_{6}$ & $\frac{C_{2} B_{1}-B_{2} G_{1}}{C_{1} C_{2}-G_{1} G_{2}}$ & $\frac{C_{1} B_{2}-B_{1} G_{2}}{C_{1} C_{2}-G_{1} G_{2}}$ & 0 & 0 & $\mathbf{B Z} \cdot \mathbf{B Z}$ \\
\hline $\mathbf{F P}_{7}$ & $\frac{C_{2} B_{1}-B_{2} G_{1}}{C_{1}^{\prime} C_{2}-G_{1} G_{2}}$ & $\frac{C_{1}^{\prime} B_{2}-B_{1} G_{2}}{C_{1}^{\prime} C_{2}-G_{1} G_{2}}$ & $\frac{F_{1}}{E_{1}} \alpha_{1}$ & 0 & $\mathbf{G Y} \cdot \mathbf{B Z}$ \\
\hline $\mathrm{FP}_{8}$ & $\frac{C_{2}^{\prime} B_{1}-B_{2} G_{1}}{C_{1} C_{2}^{\prime}-G_{1} G_{2}}$ & $\frac{C_{1} B_{2}-B_{1} G_{2}}{C_{1} C_{2}^{\prime}-G_{1} G_{2}}$ & 0 & $\frac{F_{2}}{E_{2}} \alpha_{2}$ & $\mathbf{B Z} \cdot \mathbf{G Y}$ \\
\hline $\mathrm{FP}_{9}$ & $\frac{C_{2}^{\prime} B_{1}^{2}-B_{2} G_{1}}{C_{1}^{\prime} C_{2}^{\prime}-G_{1} G_{2}}$ & $\frac{C_{1}^{\prime} B_{2}^{2}-B_{1} G_{2}}{C_{1}^{\prime} C_{2}^{\prime}-G_{1} G_{2}}$ & $\frac{F_{1}}{E_{1}} \alpha_{1}$ & $\frac{F_{2}}{E_{2}} \alpha_{2}$ & $\mathbf{G Y} \cdot \mathbf{G Y}$ \\
\hline
\end{tabular}
product of two Banks-Zaks type fixed points. At $\mathrm{FP}_{7}$ and $\mathrm{FP}_{8}$, only one of the Yukawa couplings vanish, implying

TABLE III. The various types of fixed points in gauge-Yukawa theories with semisimple gauge group $\mathcal{G}_{1} \otimes \mathcal{G}_{2}$ and (10), (12). We also indicate how the nine qualitatively different fixed points can be interpreted as products of the Gaussian $(\mathrm{G})$, Banks-Zaks (BZ) and gauge-Yukawa (GY) fixed points as seen from the individual gauge group factors (see main text). 
that these are products of a gauge-Yukawa with a BanksZaks fixed point. Finally, at $\mathrm{FP}_{9}$, both Yukawa couplings are nonvanishing meaning that this is effectively the product of two gauge-Yukawa fixed points.

In theories where none of the fermions carries gauge charges under both gauge groups, we have that $G_{1}=0=G_{2}$. In this limit, and at the present level of approximation, the gauge sectors do not communicate with each other and the "direct product" interpretation of the fixed points as detailed above becomes exact. For the purpose of this work we will find it useful to refer to the effective "product" structure of interacting fixed points even in settings with $G_{1}, G_{2} \neq 0$. Whether any of the fixed points is factually realized in a given theory crucially depends on the explicit values of the various loop coefficients. We defer an explicit investigation for certain "minimal models" to Sec. III.

\section{Scalar couplings}

In [34], it has been established that scalar self-interactions play no role for the primary occurrence of weakly interacting fixed points in the gauge- or gauge-Yukawa sector. On the other hand, for consistency, scalar couplings must nevertheless take free or interacting fixed points on their own. The necessary and sufficent conditions for this to arise have been given in [34]. First, scalar couplings must take physical (real) fixed points. Second, the theory must display a stable ground state at the fixed point in the scalar sector. Below, we will analyse concrete models and show that both of these conditions are nonempty.

\section{E. Universal scaling exponents}

We briefly comment on the universal behavior and scaling exponents at the interacting fixed points of Table III. Scaling exponents arise as the eigenvalues $\vartheta_{i}$ of the stability matrix

$$
M_{i j}=\partial \beta_{i} /\left.\partial \alpha_{j}\right|_{*}
$$

at fixed points. Negative or positive eigenvalues correspond to relevant or irrelevant couplings respectively. They imply that couplings approach the fixed point following a powerlaw behavior in RG momentum scale,

$$
\alpha_{i}(\mu)-\alpha_{i}^{*}=\sum_{n} c_{n} V_{i}^{n}\left(\frac{\mu}{\Lambda}\right)^{\vartheta_{n}}+\text { subleading. }
$$

Classically, we have that $\vartheta \equiv 0$. Quantum-mechanically, and at a Gaussian fixed point, eigenvalues continue to vanish and the behavior of couplings is determined by higher order effects. Then couplings are either exactly marginal $\vartheta \equiv 0$ or marginally relevant $\vartheta \rightarrow 0^{-}$or marginally irrelevant $\vartheta \rightarrow 0^{+}$. In a slight abuse of language we will from now on denote relevant and marginally relevant ones as $\vartheta \leq 0$, and vice versa for irrelevant ones.

Given that the scalar couplings do not feed back to the gauge-Yukawa sector at the leading non-trivial order in perturbation theory, we may neglect them for a discussion of the eigenvalue spectrum

$$
\left\{\vartheta_{i}, i=1, \cdots 4\right\}
$$

related to the two gauge and Yukawa couplings. The fixed point $\mathrm{FP}_{1}$ is Gaussian in all couplings, and the scaling of couplings are either marginally relevant or marginally irrelevant. Only if $B_{i}>0$ trajectories can emanate from the Gaussian, meaning that it is a UV fixed point iff the theory is asymptotically free in both couplings. Furthermore, asymptotic freedom in the gauge couplings entails asymptotic freedom in the Yukawa couplings leading to four marginally relevant couplings with eigenvalues

$$
\vartheta_{1}, \vartheta_{2}, \vartheta_{3}, \vartheta_{4} \leq 0
$$

The fixed points $\mathrm{FP}_{2}$ and $\mathrm{FP}_{3}$ are products of a Banks-Zaks in one gauge sector with a Gaussian fixed point in the other. Scaling exponents are then of the form

$$
\vartheta_{1}, \vartheta_{2}, \vartheta_{3} \leq 0<\vartheta_{4}
$$

provided the gauge sector with Gaussian fixed point is asymptotically free. For IR free gauge coupling, we instead have the pattern

$$
\vartheta_{1}<0 \leq \vartheta_{2}, \vartheta_{3}, \vartheta_{4}
$$

At the fixed points $\mathrm{FP}_{4}$ and $\mathrm{FP}_{5}$, the theory is the product of a Gaussian and a gauge-Yukawa fixed point. Consequently, four possibilities arise: Provided that the theory is asymptotically safe at the gauge-Yukawa fixed point and asymptotically or infrared free at the Gaussian, scaling exponents are of the form (17) or (18), respectively. Conversely, if the gauge Yukawa fixed point is IR, the eigenvalue spectrum reads

$$
\vartheta_{1}, \vartheta_{2} \leq 0 \leq \vartheta_{3}, \vartheta_{4}
$$

if the Gaussian is asymptotically free. Finally, if the Gaussian is IR free and the gauge-Yukawa fixed point IR, all couplings are UV irrelevant and

$$
0 \leq \vartheta_{1}, \vartheta_{2}, \vartheta_{3}, \vartheta_{4}
$$

More work is required to determine the scaling exponents at the fully interacting fixed points $\mathrm{FP}_{6}-\mathrm{FP}_{9}$. To that end, we write the characteristic polynomial of the stability matrix as 


$$
\sum_{n=0}^{4} T_{n} \vartheta^{n}=0
$$

The coefficients $T_{n}$ are functions of the loop coefficients. Introducing $B=\left|B_{1}\right|$ and $B_{2}=P B_{1}$, with $P$ some free parameter, we can make a scaling analysis in the limit $B \ll 1$. Normalizing the coefficient $T_{4}$ to unity, $T_{4}=1$, it then follows from the structure of the beta functions that $T_{0}=\mathcal{O}\left(B^{6}\right), T_{1}=\mathcal{O}\left(B^{4}\right), T_{2}=\mathcal{O}\left(B^{2}\right)$ and $T_{3}=\mathcal{O}(B)$ to leading order in $B$. In the limit where $B \ll 1$ we can deduce exact closed expressions for the leading order behaviour of the eigenvalues from solutions to two quadratic equations,

$$
\begin{aligned}
& 0=\vartheta^{2}+T_{3} \vartheta+T_{2} \\
& 0=T_{2} \vartheta^{2}+T_{1} \vartheta+T_{0} .
\end{aligned}
$$

The general expressions are quite lengthy and shall not be given here explicitly. We note that the four eigenvalues of the four couplings at the four fully interacting fixed points $\mathrm{FP}_{6}-\mathrm{FP}_{9}$ are the four solutions to (22). Irrespective of their signs, and barring exceptional numerical cancellations, we conclude that two scaling exponents are quadratic and two are linear in $B$,

$$
\begin{aligned}
& \vartheta_{1,2}=-\frac{1}{2}\left(T_{3} \pm \sqrt{T_{3}^{2}-4 T_{2}}\right)=\mathcal{O}\left(B^{2}\right) \\
& \vartheta_{3,4}=-\frac{1}{2 T_{2}}\left(T_{1} \pm \sqrt{T_{1}^{2}-4 T_{0} T_{2}}\right)=\mathcal{O}(B) .
\end{aligned}
$$

This is reminiscent of fixed points in gauge-Yukawa theories with a simple gauge group. The main reason for the appearance of two eigenvalues of order $\mathcal{O}\left(B^{2}\right)$ relates to the gauge sector, where the interacting fixed point arises through the cancellation at two-loop level. Conversely, two eigenvalues of order $\mathcal{O}(B)$ relate to the Yukawa couplings, as they arise from a cancellation at one-loop level. This completes the discussion of fixed points in general weakly coupled semisimple gauge theories.

\section{MINIMAL MODELS}

In this section we introduce in concrete terms a family of semisimple gauge theories whose interacting fixed points will be analyzed exactly within perturbation theory in the Veneziano limit.

\section{A. Semisimple gauge theory}

We consider families of massless four-dimensional quantum field theories with a semisimple gauge group

$$
S U\left(N_{\mathrm{C}}\right) \times S U\left(N_{\mathrm{c}}\right)
$$

for general non-Abelian factors with $N_{\mathrm{C}} \geq 2$ and $N_{\mathrm{c}} \geq 2$.

\begin{tabular}{|c|c|c|c|c|c|c|c|}
\hline \multirow[b]{2}{*}{ Representation } & \multicolumn{3}{|c|}{ Fermions } & \multicolumn{2}{|c|}{ Scalars } & \multicolumn{2}{|c|}{ Gauge fields } \\
\hline & $Q$ & $q$ & $\psi$ & $H$ & $h$ & $A_{\mu}$ & $a_{\mu}$ \\
\hline Under $S U\left(N_{C}\right)$ & $N_{\mathrm{C}}$ & 1 & $N_{\mathrm{C}}$ & 1 & 1 & $N_{\mathrm{C}}^{2}-1$ & 1 \\
\hline Under $S U\left(N_{c}\right)$ & 1 & $N_{\mathrm{c}}$ & $N_{\mathrm{c}}$ & 1 & 1 & 1 & $N_{\mathrm{c}}^{2}-1$ \\
\hline Multiplicity & $N_{\mathrm{F}}$ & $N_{\mathrm{f}}$ & $N_{\psi}$ & $N_{\mathrm{F}}^{2}$ & $N_{\mathrm{f}}^{2}$ & 1 & 1 \\
\hline
\end{tabular}
Specifically, our models contains $S U\left(N_{\mathrm{C}}\right)$ gauge fields $A_{\mu}$
TABLE IV. Representation under the semisimple gauge symmetry (24) together with flavor multiplicities of all fields. Gauge (fermion) fields are either in the adjoint (fundamental) or trivial representation.

with field strength $F_{\mu \nu}$, and $S U\left(N_{\mathrm{c}}\right)$ gauge fields $a_{\mu}$ with field strength $f_{\mu \nu}$. The gauge fields are coupled to $N_{\mathrm{F}}$ flavors of fermions $Q_{i}, N_{\mathrm{f}}$ flavors of fermions $q_{i}$, and $N_{\psi}$ flavors of fermions $\psi_{i}$. The fermions $(Q, q, \psi)$ transform in the fundamental representation of the first, the second, and both gauge group(s) (24), respectively, as summarized in Table IV. The Dirac fermions $\psi$ are responsible for the semisimple character of the theory and serve as messengers to communicate between gauge sectors. All fermions are Dirac to guarantee anomaly cancellation. The fermions $(Q, q)$ additionally couple via Yukawa interactions to an $N_{\mathrm{F}} \times N_{\mathrm{F}}$ matrix scalar field $H$ and an $N_{\mathrm{f}} \times N_{\mathrm{f}}$ matrix scalar field $h$, respectively. The scalars $H$ and $h$ are invariant under $U\left(N_{\mathrm{F}}\right)_{L} \times U\left(N_{\mathrm{F}}\right)_{R}$ and $U\left(N_{\mathrm{f}}\right)_{L} \times U\left(N_{\mathrm{f}}\right)_{R}$ global flavor rotations, respectively, and singlets under the gauge symmetry. They can be viewed as elementary mesons in that they carry the same global quantum numbers as the singlet scalar bound states $\sim\langle Q \bar{Q}\rangle$ and $\sim\langle q \bar{q}\rangle$. The fermions $\psi$ are not furnished with Yukawa interactions.

The fundamental action is taken to be the sum of the individual Yang-Mills actions, the fermion kinetic terms, the Yukawa interactions, and the scalar kinetic and selfinteraction Lagrangeans $L=L_{\mathrm{YM}}+L_{F}+L_{Y}+L_{S}+L_{\text {pot }}$, with

$$
\begin{aligned}
L_{\mathrm{YM}} & =-\frac{1}{2} \operatorname{Tr} F^{\mu \nu} F_{\mu \nu}-\frac{1}{2} \operatorname{Tr} f^{\mu \nu} f_{\mu \nu} \\
L_{F} & =\operatorname{Tr}(\bar{Q} i \not D Q)+\operatorname{Tr}(\bar{q} i \not D q)+\operatorname{Tr}(\bar{\psi} i \not \emptyset \psi) \\
L_{Y} & =Y \operatorname{Tr}\left(\bar{Q}_{L} H Q_{R}+\bar{Q}_{R} H^{\dagger} Q_{L}\right)+y \operatorname{Tr}\left(\bar{q}_{L} h q_{R}+\bar{q}_{R} h^{\dagger} q_{L}\right) \\
L_{S} & =\operatorname{Tr}\left(\partial_{\mu} H^{\dagger} \partial^{\mu} H\right)+\operatorname{Tr}\left(\partial_{\mu} h^{\dagger} \partial^{\mu} h\right) \\
L_{\mathrm{pot}} & =-U \operatorname{Tr}\left(H^{\dagger} H\right)^{2}-V\left(\operatorname{Tr} H^{\dagger} H\right)^{2} \\
& -u \operatorname{Tr}\left(h^{\dagger} h\right)^{2}-v\left(\operatorname{Tr} h^{\dagger} h\right)^{2}-w \operatorname{Tr} H^{\dagger} H \operatorname{Tr} h^{\dagger} h
\end{aligned}
$$

The trace $\operatorname{Tr}$ denotes the trace over both color and flavor indices, and the decomposition $Q=Q_{L}+Q_{R}$ with $Q_{L / R}=\frac{1}{2}\left(1 \pm \gamma_{5}\right) Q$ is understood for all fermions $Q$ and $q$. Mass terms are neglected at the present stage as their effect is subleading to the main features developed below. In four dimensions, the theory is renormalizable in perturbation theory. 
The theory has nine classically marginal coupling constants given by the two gauge couplings, the two Yukawa couplings, and five quartic scalar couplings. We write them as

$\alpha_{1}=\frac{g_{1}^{2} N_{\mathrm{C}}}{(4 \pi)^{2}}, \quad \alpha_{2}=\frac{g_{2}^{2} N_{\mathrm{c}}}{(4 \pi)^{2}}, \quad \alpha_{Y}=\frac{Y^{2} N_{\mathrm{C}}}{(4 \pi)^{2}}, \quad \alpha_{y}=\frac{y^{2} N_{\mathrm{c}}}{(4 \pi)^{2}}$,

$\alpha_{U}=\frac{u N_{\mathrm{F}}}{(4 \pi)^{2}}, \quad \alpha_{V}=\frac{v N_{\mathrm{F}}^{2}}{(4 \pi)^{2}}, \quad \alpha_{u}=\frac{u N_{\mathrm{f}}}{(4 \pi)^{2}}, \quad \alpha_{v}=\frac{v N_{\mathrm{f}}^{2}}{(4 \pi)^{2}}$,

where we have normalized the couplings with the appropriate loop factor and powers of $N_{C}, N_{c}, N_{\mathrm{F}}$ and $N_{\mathrm{f}}$ in view of the Veneziano limit to be adopted below. Notice the additional power of $N_{\mathrm{F}}$ and $N_{\mathrm{f}}$ in the definitions of the scalar double-trace couplings. We normalize the quartic "portal" coupling as

$$
\alpha_{w}=\frac{w N_{\mathrm{F}} N_{\mathrm{f}}}{(4 \pi)^{2}} .
$$

It is responsible for a mixing amongst the scalar sectors starting at tree level. Below, we use the shorthand notation $\beta_{i} \equiv \partial_{t} \alpha_{i}$ with $i=(1,2, Y, y, U, u, V, v, w)$ to indicate the $\beta$-functions for the couplings (26). To obtain explicit expressions for these, we exploit the formal results summarized in [41-43]. The semisimple character of the theory is switched off if the $N_{\psi}$ messenger fermions (which carry charges under both gauge groups) are replaced by $N_{1}$ and $N_{2}$ Yukawa-less fermions in the fundamental of $S U\left(N_{C}\right)$ and $S U\left(N_{c}\right)$, respectively, with

$$
N_{1}=N_{c} N_{\psi}, \quad N_{2}=N_{C} N_{\psi} .
$$

If in addition $\alpha_{w}=0$, the theories (25) reduce to a "direct product" of simple gauge Yukawa theories with (28). Also, in the limit where one of the gauge groups is switched off, $\alpha_{1} \equiv 0$ ( or $\alpha_{2} \equiv 0$ ), one gauge sector and the scalars decouples straightaway, and we are left with a simple gauge theory. Finally, if $N_{1}=0=N_{2}$, we recover the models of [32] in each gauge sector (displaying asymptotic safety for certain field multiplicities). Below, we will find it useful to contrast results with those from the "direct product" limit.

\section{B. Free parameters and Veneziano limit}

We now discuss the set of fundamentally free parameters of our models. On the level of the Lagrangian, the free parameters of the theory are the matter field multiplicities

$$
N_{\mathrm{C}}, \quad N_{\mathrm{c}}, \quad N_{\mathrm{F}}, \quad N_{\mathrm{f}}, \quad N_{\psi} .
$$

Notice that the $N_{\psi}$ fermions $\psi$ are centrally responsible for interactions between the gauge sectors. In the limit

$$
N_{\psi}=0
$$

the interaction between gauge sectors reduces to effects mediated by the portal coupling $\alpha_{w} \neq 0$, which are strongly loop-suppressed. In this limit, results for fixed points and running couplings fall back to those for the individual gauge sectors [32]. Results for fixed points for general $N_{\psi}$ are deferred to Appendix A. Here, we will set $N_{\psi}$ to a finite value,

$$
N_{\psi}=1
$$

This leaves us with four free parameters. In order to achieve exact perturbativity, we perform a Veneziano limit [49] by sending the number of colors and the number of flavors $\left(N_{\mathrm{C}}, N_{\mathrm{c}}, N_{\mathrm{F}}, N_{\mathrm{f}}\right)$ to infinity but keeping their ratios fixed. This reduces the set of free parameters of the model down to three, which we chose to be

$$
R=\frac{N_{\mathrm{c}}}{N_{\mathrm{C}}}, \quad S=\frac{N_{\mathrm{F}}}{N_{\mathrm{C}}}, \quad T=\frac{N_{\mathrm{f}}}{N_{\mathrm{c}}} .
$$

The ratio

$$
F=\frac{N_{\mathrm{f}}}{N_{\mathrm{F}}}
$$

is then no longer a free parameter, but fixed as $F=R T / S$ from (32). By their very definiton, the parameters (32) are positive semidefinite and can take values $0 \leq F, R$, $S, T \leq \infty$. However, we will see below that their values are further constrained if we impose perturbativity for all couplings.

\section{Perturbativity to leading order}

The RG evolution of couplings is analyzed within the perturbative loop expansion. To leading order (LO), the running of the gauge couplings reads $\beta_{i}=-B_{i} \alpha_{i}^{2}$ (no sum), with $B_{i}$ the one-loop gauge coefficients for the gauge coupling $\alpha_{i}$. In the Veneziano limit, the one-loop coefficients take the form

$$
B_{i}=-\frac{4}{3} \epsilon_{i} .
$$

In terms of (32) and in the Veneziano limit, the parameters $\epsilon_{i}$ are given by

$$
\epsilon_{1}=S+R-\frac{11}{2}, \quad \epsilon_{2}=T+\frac{1}{R}-\frac{11}{2} .
$$

We can therefore trade the free parameters $(S, T)$ defined in (32) for $\left(\epsilon_{1}, \epsilon_{2}\right)$ and consider the set

$$
\left(\epsilon_{1}, \epsilon_{2}, R\right)
$$


as free parameters which characterize the matter content of the theory. Under the exchange of gauge groups we have

$$
\left(\epsilon_{1}, \epsilon_{2}, R\right) \rightarrow\left(\epsilon_{2}, \epsilon_{1}, R^{-1}\right) .
$$

For fixed $R$, we observe that $R-\frac{11}{2} \leq \epsilon_{1}<\infty$ and $1 / R-\frac{11}{2} \leq \epsilon_{2}<\infty$. Perturbativity in either of the gauge couplings requires that both one-loop coefficients $B_{i}$ are parametrically small compared to unity. Therefore we impose

$$
0<\left|\epsilon_{i}\right| \ll 1
$$

This requirement of exact perturbativity in both gauge sectors entails the important constraint

$$
\frac{2}{11}<R<\frac{11}{2}
$$

Outside of this range, no physical values for $S$ and $T$ can be found such that (38) holds true. Inside this range, physical values are constrained within $0 \leq S, T \leq \frac{11}{2}-\frac{2}{11}$. The parameters (36) have a simple interpretation. The small paramaters $\epsilon_{i}$ control the perturbativity within each of the gauge sectors, whereas the parameter $R$ controls the "interactions" between the two gauge sectors. It is the presence of $R$ which makes these theories intrinsically semisimple, rather than being the direct product of two simple gauge theories. Perturbativity is no longer required in the limit where one of the gauge sectors is switched off, and the constraint (39) is relaxed into

$$
\begin{gathered}
0 \leq R<\frac{11}{2} \quad \text { if } \alpha_{2}^{*} \equiv 0, \\
\frac{2}{11}<R<\infty \quad \text { if } \alpha_{1}^{*} \equiv 0 .
\end{gathered}
$$

The parametrization (36) is most convenient for expressing the relevant RG beta functions for all couplings.

Finally, for some of the subsequent considerations we replace the two small parameters $\left(\epsilon_{1}, \epsilon_{2}\right)$ by $(\epsilon, P)$, a single small parameter $\epsilon$ proportional to $\epsilon_{1}$ together with a parameter $P$ related to the ratio between $\epsilon_{1}$ and $\epsilon_{2}$. Specifically, we introduce

$$
\epsilon_{1}=R \epsilon, \quad \epsilon_{2}=P \frac{\epsilon}{R}
$$

which is equivalent to $P=R^{2} \epsilon_{2} / \epsilon_{1}$ together with $\epsilon=\epsilon_{1} / R$ and $\epsilon=P R \epsilon_{2}{ }^{2}$ Since $R$ can only take finite positive values,

\footnotetext{
${ }^{2}$ The choice (41) can be motivated by dimensional analysis of (35) which shows that $\epsilon_{1}$ and $\epsilon_{2}$ formally scale as $\sim R$ and $\sim 1 / R$ for large or small $R$, respectively, whereby their ratio $\epsilon_{1} / \epsilon_{1}$ scales as $\sim R^{2}$. The large- $R$ behavior is factored-out by our parametrization.
}

the additional rescaling with $R$ does not affect the relative sign between $\epsilon_{1}$ and $\epsilon$. In this manner we have traded the free parameters $\left(\epsilon_{1}, \epsilon_{2}, R\right)$ for

$$
(R, P, \epsilon) \text {. }
$$

Notice that the parameter $P$ can be expressed as

$$
P=\frac{1+\left(N_{\mathrm{f}}-\frac{11}{2} N_{\mathrm{c}}\right) / N_{\mathrm{C}}}{1+\left(N_{\mathrm{F}}-\frac{11}{2} N_{\mathrm{C}}\right) / N_{\mathrm{c}}}
$$

in terms of the field multiplicities (29). It thus may take any real value of either sign with $-\infty<P<\infty$, whereas $R$ must take values within the range (39). Moreover,

$$
\epsilon=1+\frac{N_{\mathrm{F}}-\frac{11}{2} N_{\mathrm{C}}}{N_{\mathrm{c}}} .
$$

In this parametrization, the ratio of fermion flavor multiplicities (33) becomes

$F=\frac{11 R-2}{11-2 R}+\frac{2 R}{11-2 R}\left(\frac{P}{R}-\frac{11 R-2}{11-2 R}\right) \epsilon+\mathcal{O}\left(\epsilon^{2}\right)$.

We also observe that the substitution

$$
(R, P, \epsilon) \rightarrow\left(R^{-1}, P^{-1}, P \epsilon\right)
$$

relates to the exchange of gauge groups. The parametrization (42) is most convenient for analysing the various interacting fixed points and their scaling exponents (see below). This completes the definition of our models.

\section{Anomalous dimensions}

We provide results for the anomalous dimensions associated to the fermions and scalars. Furthermore, if mass terms are present, their renormalization is induced through the RG flow of the gauge, Yukawa, and scalar couplings. Following [32], we define the scalar anomalous dimensions as $\Delta_{S}=1+\gamma_{S}$, where $\gamma_{S} \equiv \frac{1}{2} d \ln Z_{S} / d \ln \mu$ and $S=H, h$. Within perturbation theory, the one and two loop contributions read

$$
\begin{aligned}
& \gamma_{H}=\alpha_{Y}-\frac{3}{2}\left(\frac{11}{2}-\epsilon_{1}-R\right) \alpha_{Y}^{2}+\frac{5}{2} \alpha_{Y} \alpha_{1}+2 \alpha_{U}^{2}+\mathcal{O}\left(\alpha^{3}\right), \\
& \gamma_{h}=\alpha_{y}-\frac{3}{2}\left(\frac{11}{2}+\epsilon_{2}-\frac{1}{R}\right) \alpha_{y}^{2}+\frac{5}{2} \alpha_{y} \alpha_{2}+2 \alpha_{u}^{2}+\mathcal{O}\left(\alpha^{3}\right) .
\end{aligned}
$$

For the fermion anomalous dimensions $\gamma_{F} \equiv d \ln Z_{F} / d \ln \mu$ with $F=Q, q, \psi$, we find 


$$
\begin{aligned}
& \gamma_{Q}=\left(\frac{11}{2}+\epsilon_{1}-R\right) \alpha_{Y}+\xi_{1} \alpha_{1}+\mathcal{O}\left(\alpha^{2}\right), \\
& \gamma_{q}=\left(\frac{11}{2}+\epsilon_{2}-\frac{1}{R}\right) \alpha_{Y}+\xi_{2} \alpha_{2}+\mathcal{O}\left(\alpha^{2}\right), \\
& \gamma_{\psi}=\xi_{1} \alpha_{1}+\xi_{2} \alpha_{2}+\mathcal{O}\left(\alpha^{2}\right),
\end{aligned}
$$

where $\xi_{1}$ and $\xi_{2}$ denote the gauge fixing parameters for the first and second gauge group respectively.

The anomalous dimension for the scalar mass terms can be derived from the composite operator $\sim M^{2} \operatorname{Tr} H^{\dagger} H$ and $\sim m^{2} \operatorname{Tr} h^{\dagger} h$. Introducing the mass anomalous dimension $\gamma_{M}=d \ln M^{2} / d \ln \mu$, and similarly for $m$, one finds

$$
\begin{aligned}
& \gamma_{M}=8 \alpha_{U}+4 \alpha_{V}+2 \alpha_{Y}+\mathcal{O}\left(\alpha^{2}\right) \\
& \gamma_{m}=8 \alpha_{u}+4 \alpha_{v}+2 \alpha_{y}+\mathcal{O}\left(\alpha^{2}\right),
\end{aligned}
$$

to one-loop order. We also compute the running of the mass terms for the scalars

$$
\begin{aligned}
& \beta_{M^{2}}=\gamma_{M} M^{2}+2 F m^{2} \alpha_{w}+\mathcal{O}\left(\alpha^{2}, \alpha m_{F}^{2}\right), \\
& \beta_{m^{2}}=\gamma_{m} m^{2}+2 F^{-1} M^{2} \alpha_{w}+\mathcal{O}\left(\alpha^{2}, \alpha m_{F}^{2}\right),
\end{aligned}
$$

where the parameter $F \equiv N_{\mathrm{f}} / N_{\mathrm{F}}$ solely depends on $R$ to leading order in $\epsilon$, see (45). Notice that the coupling $\alpha_{w}$ induces a mixing amongst the different scalar masses already at one-loop level.

Analogously, the anomalous dimension for the fermion mass operator is defined as $\Delta_{F}=3+\gamma_{M_{F}}$ with $\gamma_{M_{F}} \equiv d \ln M_{F} / d \ln \mu$, and $M_{F}$ stands for one of the fermion masses with $F=Q, q$ or $\psi$. Within perturbation theory, the one loop contributions read

$$
\begin{aligned}
& \gamma_{M_{Q}}=\alpha_{Y}\left(\frac{13}{2}+\epsilon_{1}-R\right)-3 \alpha_{1}-+\mathcal{O}\left(\alpha^{2}\right), \\
& \gamma_{M_{q}}=\alpha_{y}\left(\frac{13}{2}+\epsilon_{2}-\frac{1}{R}\right)-3 \alpha_{2}+\mathcal{O}\left(\alpha^{2}\right) \\
& \gamma_{M_{\psi}}=-3\left(\alpha_{1}+\alpha_{2}\right)+\mathcal{O}\left(\alpha^{2}\right) .
\end{aligned}
$$

For the fermion masses we have the running

$$
\beta_{m_{Q}}=\gamma_{M_{Q}} m_{Q}, \quad \beta_{m_{q}}=\gamma_{M_{q}} m_{q}, \quad \beta_{m_{\psi}}=\gamma_{M_{\psi}} m_{\psi} .
$$

We note that $\gamma_{M_{\psi}}$ is manifestly negative. For $\gamma_{M_{Q}}$ and $\gamma_{M_{q}}$ we observe that the gauge and Yukawa contributions arise with manifestly opposite signs in the parameter regime (38), (39). Hence either of these may take either sign, depending on whether the gauge or Yukawa contributions dominate.

\section{E. Running couplings beyond the leading order}

We now go beyond the leading order in perturbation theory and provide the complete, minimal set of RG equations which display exact and weakly interacting fixed points. To that end, we must retain terms up to two loop order in the gauge coupling, or else an interacting fixed point cannot arise. At the same time, in order to explore the feasibility of asymptotically safe UV fixed points we must retain the Yukawa couplings [34], minimally at the leading nontrivial order which is one loop. Following [32] we refer to this level of approximation in the gauge-Yukawa sector as next-to-leading order (NLO). In the presence of scalar fields, we also must retain the quartic scalar couplings at their leading nontrivial order. We refer to this approximation of the gauge-Yukawa-scalar sector as $\mathrm{NLO}^{\prime}$ [48], see Table II. This is the minimal order in perturbation theory at which a fully interacting fixed point can be determined in all couplings with canonically vanishing mass dimension.

In general, the RG flow for the gauge and Yukawa couplings at $\mathrm{NLO}^{\prime}$ is strictly independent of the scalar couplings owing to the fact that scalar loops only arise starting from the two loop order in the Yukawa sector, and at three (four) loop order in the gauge sector, if the scalars are charged (uncharged). Furthermore, the scalar sector at $\mathrm{NLO}^{\prime}$ depends on the Yukawa couplings, but not on the gauge couplings owing to the fact that the scalars are uncharged. Consequently, we observe a partial decoupling of the gauge-Yukawa sector $\left(\alpha_{1}, \alpha_{2}, \alpha_{Y}, \alpha_{y}\right)$ and the scalar sector $\left(\alpha_{U}, \alpha_{V}, \alpha_{u}, \alpha_{v}, \alpha_{w}\right)$. This structure will be exploited systematically below to identify all interacting fixed points.

We begin with the gauge-Yukawa sector where we find the coupled beta functions (10) which are characterized by ten loop coefficients $C_{i}, D_{i}, E_{i}, F_{i}$ and $G_{i}(i=1,2)$, together with the coefficients $B_{i}$ given in (34) or, equivalently, the perturbative control parameters (35). The oneloop coefficients arise in the Yukawa sector and take the values

$$
\begin{array}{ll}
E_{1}=13+2\left(\epsilon_{1}-R\right), & F_{1}=6, \\
E_{2}=13+2\left(\epsilon_{2}-\frac{1}{R}\right), & F_{2}=6 .
\end{array}
$$

At the two-loop level we have six coefficients related to the gauge, Yukawa, and mixing contribution, which are found to be

$$
\begin{array}{lll}
C_{1}=25+\frac{26}{3} \epsilon_{1}, & D_{1}=2\left(\epsilon_{1}-R+\frac{11}{2}\right)^{2}, & G_{1}=2 R \\
C_{2}=25+\frac{26}{3} \epsilon_{2}, & D_{2}=2\left(\epsilon_{2}-\frac{1}{R}+\frac{11}{2}\right)^{2}, & G_{2}=\frac{2}{R}
\end{array}
$$


A few comments are in order. First, the loop coefficients $D_{i}, E_{i}, F_{i}, G_{i}>0$ as they must for any quantum field theory. Additionally we confirm that $C_{i}>0$ [34], provided the parameters $\epsilon_{i}$ are in the perturbative regime (38). Second, provided that $R=0$ in the expressions for $\epsilon_{1}$, $E_{1}$ and $G_{1}$, and $1 / R=0$ in those for $\epsilon_{2}, E_{2}$, and $G_{2}$, the system (10) falls back onto a direct product of simple gauge-Yukawa theories, each of the type discussed in [32]. Notice that this limit cannot be achieved parametrically in $R$. The reason for this is the presence of $N_{\psi}$ fermions which are charged under both gauge groups. They contribute with reciprocal multiplicity $R \leftrightarrow 1 / R$ to the Yukawa-induced loop terms $D_{i}$ and $E_{i}$ as well as to the mixing terms $G_{i}$. Exact decoupling of the gauge sectors then becomes visible only in the parametric limit where $N_{\psi} \rightarrow 0$ whereby all terms involving $R$ or $1 / R$ drop out. Finally, we note that the exchange of gauge groups $\mathcal{G}_{1} \leftrightarrow \mathcal{G}_{2}$ corresponds to $R \leftrightarrow 1 / R$ and $S \leftrightarrow T$, implying $\epsilon_{1} \leftrightarrow \epsilon_{2}$ and $P \leftrightarrow 1 / P$, respectively. Evidently, at the symmetric point $R=1$ and $\epsilon_{1}=\epsilon_{2}$ (or $P=1$ ) we have exact exchange symmetry between gauge group factors.

Inserting (53), (54) and (34) into the general expression (10), we obtain the perturbative RG flow for the gaugeYukawa system at NLO accuracy

$$
\begin{aligned}
\beta_{1}= & \frac{4}{3} \epsilon_{1} \alpha_{1}^{2}+\left(25+\frac{26}{3} \epsilon_{1}\right) \alpha_{1}^{3}-2\left(\epsilon_{1}-R+\frac{11}{2}\right)^{2} \alpha_{1}^{2} \alpha_{Y} \\
& +2 R \alpha_{1}^{2} \alpha_{2}, \\
\beta_{2}= & \frac{4}{3} \epsilon_{2} \alpha_{2}^{2}+\left(25+\frac{26}{3} \epsilon_{2}\right) \alpha_{2}^{3}-2\left(\epsilon_{2}-\frac{1}{R}+\frac{11}{2}\right)^{2} \alpha_{2}^{2} \alpha_{y} \\
& +\frac{2}{R} \alpha_{2}^{2} \alpha_{1}, \\
\beta_{Y}= & {\left[13+2\left(\epsilon_{1}-R\right)\right] \alpha_{Y}^{2}-6 \alpha_{Y} \alpha_{1}, } \\
\beta_{y}= & {\left[13+2\left(\epsilon_{2}-\frac{1}{R}\right)\right] \alpha_{y}^{2}-6 \alpha_{y} \alpha_{2} . }
\end{aligned}
$$

We observe that the running of Yukawa couplings at one loop is determined by the fermion mass anomalous dimension (51),

$$
\beta_{Y}=2 \gamma_{M_{Q}} \alpha_{Y}, \quad \beta_{y}=2 \gamma_{M_{q}} \alpha_{y} .
$$

The result for the mass anomalous dimensions (51) can also be derived diagrammatically from the flow of the Yukawa vertices (55), thus offering an independent confirmation for the link (56).

Next, we turn to the scalar sector and the running of quartic couplings to leading order in perturbation theory, which is one loop. At $\mathrm{NLO}^{\prime}$ accuracy, we have (55) together with the beta functions for the quartic scalar couplings which are found to be
$\beta_{U}=-\left[11+2\left(\epsilon_{1}-R\right)\right] \alpha_{Y}^{2}+4 \alpha_{U}\left(\alpha_{Y}+2 \alpha_{U}\right)$,

$\beta_{V}=12 \alpha_{U}^{2}+4 \alpha_{V}\left(\alpha_{V}+4 \alpha_{U}+\alpha_{Y}\right)+\alpha_{w}^{2}$,

$\beta_{u}=-\left[11+2\left(\epsilon_{2}-\frac{1}{R}\right)\right] \alpha_{y}^{2}+4 \alpha_{u}\left(\alpha_{y}+2 \alpha_{u}\right)$,

$\beta_{v}=12 \alpha_{u}^{2}+4 \alpha_{v}\left(\alpha_{v}+4 \alpha_{u}+\alpha_{y}\right)+\alpha_{w}^{2}$,

$\beta_{w}=\alpha_{w}\left[8\left(\alpha_{U}+\alpha_{u}\right)+4\left(\alpha_{V}+\alpha_{v}\right)+2\left(\alpha_{Y}+\alpha_{y}\right)\right]$.

Their structure is worth a few remarks: First, in the Veneziano limit, $\beta_{w}$ contains no term quadratic in the coupling $\alpha_{w}$ as the coefficient is of the order $\mathcal{O}\left(N_{\mathrm{F}}^{-1} N_{\mathrm{f}}^{-1}\right)$ and suppressed by inverse powers in flavour multiplicities. Second, we notice that $\beta_{w}$ comes out proportional to $\alpha_{w}$. Consequently, $\alpha_{w}$ is a technically natural coupling according to the rationale of [50], unlike all the other quartic interactions, implying that

$$
\alpha_{w}^{*}=0
$$

constitutes an exact fixed point of the theory. Comparison with (49) shows that the proportionality factor is the sum of the scalar mass anomalous dimensions, $\beta_{w}=\alpha_{w}\left(\gamma_{M}+\gamma_{m}\right)$. The quartic coupling $\alpha_{w}$ would be promoted to a free parameter characterizing a line of fixed points with exactly marginal scaling provided that its beta function vanishes identically at one loop. This would require the vanishing of the sum of scalar anomalous mass dimensions at the fixed point,

$$
\gamma_{M}^{*}+\gamma_{m}^{*}=0
$$

Below, however, we will establish that such scenarios are incompatible with vacuum stability (see Sec. V). Moreover, at interacting fixed points we invariably find that

$$
\gamma_{M}^{*}+\gamma_{m}^{*}>0
$$

as a consequence of vacuum stability. This implies that $\alpha_{w}$ constitutes an infrared free coupling at any interacting fixed point with a stable ground state. For the purpose of the present study, we therefore limit ourselves to fixed points with (58). We then observe that the running of the remaining scalar couplings is solely fuelled by the Yukawa couplings. Furthermore, the scalar subsectors associated to the different gauge groups are disentangled in our approximation. ${ }^{3}$ Interestingly, the beta functions for $\left(\alpha_{U}, \alpha_{V}\right)$ and $\left(\alpha_{u}, \alpha_{v}\right)$ are related by the substitution $R \leftrightarrow 1 / R$ and $\epsilon_{1} \leftrightarrow \epsilon_{2}$. Moreover, the double trace scalar couplings do not couple back into any of the other couplings and their fixed points are entirely dictated by the corresponding single trace scalar and the Yukawa

\footnotetext{
${ }^{3}$ The degeneracy is lifted as soon as the quartic coupling $\alpha_{w} \neq 0$, see (25), (26).
} 
TABLE V. Gauge and Yukawa couplings at interacting fixed points following Table III to the leading order in $\epsilon$ and in terms of $(R, P, \epsilon)$. Valid domains for $(\epsilon, P, R)$ in $(61)$ are detailed in Tables VII, VIII.

\begin{tabular}{|c|c|c|c|}
\hline Fixed point & Gauge couplings & Yukawa couplings & Type \\
\hline$\overline{F_{1}}$ & $\alpha_{1}^{*}=0, \alpha_{2}^{*}=0$ & $\alpha_{Y}^{*}=0, \alpha_{y}^{*}=0$ & $G \cdot \mathbf{G}$ \\
\hline $\mathbf{F P}_{2}$ & $\alpha_{1}^{*}=-\frac{4}{75} R \epsilon, \alpha_{2}^{*}=0$, & $\alpha_{Y}^{*}=0, \alpha_{y}^{*}=0$ & $\mathbf{B Z} \cdot \mathbf{G}$ \\
\hline $\mathbf{F P}_{3}$ & $\alpha_{1}^{*}=0, \alpha_{2}^{*}=-\frac{4}{75} \frac{P \epsilon}{R}$ & $\alpha_{Y}^{*}=0, \alpha_{y}^{*}=0$ & $\mathbf{G} \cdot \mathbf{B Z}$ \\
\hline $\mathbf{F P}_{4}$ & $\alpha_{1}^{*}=\frac{2}{3} \frac{(13-2 R) R E}{(2 R-1)(3 R-19)}, \alpha_{2}^{*}=0$ & $\alpha_{Y}^{*}=\frac{4 R \epsilon}{(2 R-1)(3 R-19)}, \alpha_{y}^{*}=0$ & $\mathbf{G Y} \cdot \mathbf{G}$ \\
\hline $\mathbf{F P}_{5}$ & $\alpha_{1}^{*}=0, \alpha_{2}^{*}=\frac{2}{3} \frac{(13-2 / R)}{(2 / R-1)(3 / R-19)} \frac{P \epsilon}{R}$, & $\alpha_{Y}^{*}=0, \alpha_{y}^{*}=\frac{4 P_{\epsilon} / R}{(2 / R-1)(3 / R-19)}$, & $\mathbf{G} \cdot \mathbf{G Y}$ \\
\hline $\mathbf{F P}_{6}$ & $\alpha_{1}^{*}=\frac{-4(25-2 P / R)}{1863} R \epsilon, \alpha_{2}^{*}=\frac{-4(25-2 R / P)}{1863} \frac{P \epsilon}{R}$ & $\alpha_{Y}^{*}=0, \alpha_{y}^{*}=0$ & $\mathbf{B Z} \cdot \mathbf{B Z}$ \\
\hline $\mathbf{F P}_{7}$ & $\begin{array}{l}\alpha_{1}^{*}=\frac{2}{9} \frac{(13-2 R)(25-2 P / R)}{50 R^{2}-343 R+167} R \epsilon \\
\alpha_{2}^{*}=\frac{4}{9} \frac{(13-2 R) R / P+(2 R-1)(19-3 R)}{50 R^{2}-343 R+167} \frac{P \epsilon}{R}\end{array}$ & $\begin{aligned} \alpha_{Y}^{*} & =\frac{4}{3} \frac{25-2 P / R}{50 R^{2}-343 R+167} R \epsilon \\
\alpha_{y}^{*} & =0\end{aligned}$ & $\mathbf{G Y} \cdot \mathbf{B Z}$ \\
\hline $\mathbf{F P}_{8}$ & $\begin{aligned} \alpha_{1}^{*} & =\frac{4}{9} \frac{(13-2 / R) P / R+(2 / R-1)(19-3 / R)}{50 / R^{2}-343 / R+167} R \epsilon \\
\alpha_{2}^{*} & =\frac{2}{9} \frac{(13-2 / R)(25-2 R / P)}{50 / R^{2}-343 / R+167} \frac{P \epsilon}{R}\end{aligned}$ & $\begin{array}{l}\alpha_{Y}^{*}=0 \\
\alpha_{y}^{*}=\frac{4}{3} \frac{25-2 R / P}{50 / R^{2}-343 / R+167} \frac{P \epsilon}{R}\end{array}$ & $\mathbf{B Z} \cdot \mathbf{G Y}$ \\
\hline $\mathbf{F P}_{9}$ & $\begin{array}{l}\alpha_{1}^{*}=\frac{2}{9} \frac{(13-2 R)\left[(13-2 / R) P / R+\left(\frac{2}{2}-1\right)(3 / R-19)\right]}{\left(19 R^{2}-43 R+19\right)\left(2 / R^{2}-13 / R+2\right)} R \epsilon \\
\alpha_{2}^{*}=\frac{2}{9} \frac{(13-2 / R)[(13-2 R) R / P+(2 R-1)(3 R-19)]}{\left(19 / R^{2}-43 / R+19\right)\left(2 R^{2}-13 R+2\right)} \frac{P E}{R}\end{array}$ & $\begin{aligned} \alpha_{Y}^{*} & =\frac{6 \alpha_{1}^{*}}{13-2 R} \\
\alpha_{y}^{*} & =\frac{6 \alpha_{2}^{*}}{13-2 / R}\end{aligned}$ & $\mathbf{G Y} \cdot \mathbf{G Y}$ \\
\hline
\end{tabular}

coupling [32]. This structure allows for a straightforward systematic analysis of all weakly coupled fixed points of the theory to which we turn next.

\section{INTERACTING FIXED POINTS}

In this section, we present our results for exact fixed points in the Veneziano limit, corresponding to interacting conformal field theories, and the universal scaling exponents in their vicinity.

\section{A. Parameter space}

In Table $\mathrm{V}$ we state our results for the gauge and Yukawa couplings to leading order in (38) at all fixed points, following the nomenclature of Table III. Expressions are given as functions of the parameters $(P, R, \epsilon)$,

$$
\begin{aligned}
P & =\frac{1+\left(N_{\mathrm{f}}-\frac{11}{2} N_{\mathrm{c}}\right) / N_{\mathrm{C}}}{1+\left(N_{\mathrm{F}}-\frac{11}{2} N_{\mathrm{C}}\right) / N_{\mathrm{c}}} \\
R & =\frac{N_{\mathrm{c}}}{N_{\mathrm{C}}} \\
\operatorname{sgn} \epsilon & =\operatorname{sgn}\left(N_{\mathrm{c}}+N_{\mathrm{F}}-\frac{11}{2} N_{\mathrm{C}}\right),
\end{aligned}
$$

which only depend on the matter and gauge field multiplicities (29), and $N_{\psi}=1$. Results for general $N_{\psi}$ are given in Appendix A. We also observe (39), unless stated otherwise. Constraints on the parameters $(R, P, \epsilon)$ and other information is summarized Figs. $1-5$ and in Tables VI-VIII for the various fixed points. Below, certain characteristic values $\frac{2}{11}<R_{1}<R_{2}<R_{3}<R_{4}<\frac{11}{2}$ for the parameter $R$ are of particular interest, namely

$$
\begin{aligned}
& R_{1}=\frac{343-3 \sqrt{9361}}{100} \approx 0.53, \\
& R_{2}=\frac{43-9 \sqrt{5}}{38} \approx 0.60, \\
& R_{3}=\frac{43+9 \sqrt{5}}{38} \approx 1.66, \\
& R_{4}=\frac{343+3 \sqrt{9361}}{100} \approx 1.90 .
\end{aligned}
$$

Their origin is explained in Appendix B. After these preliminaries we are in a position to analyze the fixed point spectra.

\section{B. Partially and fully interacting fixed points}

Gauge theories with (55), (57) can have two types of interacting fixed points: partially interacting ones where one gauge coupling takes the Gaussian fixed point $\left(\mathrm{FP}_{2}, \mathrm{FP}_{3}\right.$, $\mathrm{FP}_{4}, \mathrm{FP}_{5}$ ), and fully interacting ones where both gauge sectors remain interacting $\left(\mathrm{FP}_{6}, \mathrm{FP}_{7}, \mathrm{FP}_{8}, \mathrm{FP}_{9}\right)$, see Table III. At partially interacting fixed points, one gauge sector decouples and the semisimple theory with (55), (57) effectively reduces to a simple gauge theory. Simple gauge theories have three possible types of perturbative fixed points: the Gaussian $(\mathrm{G})$, the Banks-Zaks (BZ), and gauge-Yukawa (GY) fixed points for each independent linear combination of the Yukawa couplings [34]. In our setting, at $\mathrm{FP}_{2}$ and $\mathrm{FP}_{4}$ we have that $\alpha_{2}^{*} \equiv 0$, and the theory reduces to a simple gauge theory with 


$$
\begin{aligned}
& \beta_{1}=\frac{4}{3} \epsilon_{1} \alpha_{1}^{2}+\left(25+\frac{26}{3} \epsilon_{1}\right) \alpha_{1}^{3}-2\left(\epsilon_{1}-R+\frac{11}{2}\right)^{2} \alpha_{1}^{2} \alpha_{Y} \\
& \beta_{Y}=\left[13+2\left(\epsilon_{1}-R\right)\right] \alpha_{Y}^{2}-6 \alpha_{Y} \alpha_{1} \\
& \beta_{U}=-\left[11+2\left(\epsilon_{1}-R\right)\right] \alpha_{Y}^{2}+4 \alpha_{U}\left(\alpha_{Y}+2 \alpha_{U}\right), \\
& \beta_{V}=12 \alpha_{U}^{2}+4 \alpha_{V}\left(\alpha_{V}+4 \alpha_{U}+\alpha_{Y}\right),
\end{aligned}
$$

at $\mathrm{NLO}^{\prime}$ accuracy, where the parameter $R$ with

$$
0 \leq R=\frac{N_{1}}{N_{\mathrm{C}}}<\frac{11}{2}
$$

measures the number of Yukawa-less Dirac fermions $N_{1}$ in the fundamental representation in units of $N_{\mathrm{C}}$. Notice that $N_{1}$ is related to $N_{\psi} \mathrm{via}$ (28) in the theories (25). On the other hand, $N_{1}$ can be viewed as an independent parameter (counting the Yukawa-less fermions in the fundamental representation of the gauge group) if one were to switch off the semisimple character of the theory. For $R=0$ the theory (63) reduces to the one investigated in [32]. The lower bound on $R$ (39) is relaxed in (64), because the requirement of perturbativity for an interacting fixed point in the other gauge sector has become redundant. We observe the $R$-independent Banks-Zaks (BZ) fixed point $\alpha_{1}^{*}=\frac{4}{75} \epsilon_{1}$ which is, invariably, IR. To leading order in $\epsilon_{1}$ we also find a gauge-Yukawa (GY) fixed point

$\alpha_{g}^{*}=\frac{26-4 R}{57-9 R} \frac{\epsilon_{1}}{1-2 R}$

$\alpha_{Y}^{*}=\frac{4}{19-3 R} \frac{\epsilon_{1}}{1-2 R}$

$\alpha_{U}^{*}=\frac{\sqrt{23-4 R}-1}{19-3 R} \frac{\epsilon_{1}}{1-2 R}$

$\alpha_{V}^{*}=\frac{-2 \sqrt{23-4 R}+\sqrt{20-4 R+6 \sqrt{23-4 R}}}{19-3 R} \frac{\epsilon_{1}}{1-2 R}$.

For $\epsilon_{1}>0$, the GY fixed point is UV and physical as long as $0 \leq R<\frac{1}{2}$. It can be interpreted as a "deformation" of the UV fixed point analyzed in [32] owing to the presence of charged Yukawa-less fermions. Once $R>\frac{1}{2}$, however, the fixed point is physical iff $\epsilon_{1}<0$ where it becomes an IR fixed point. This new regime is entirely due to the Yukawa-less fermions and does not arise in the model of [32]. This pattern can also be read off from the scaling exponents, which, at the gauge Yukawa fixed point and to the leading nontrivial order in $\epsilon_{1}$, are given by

$$
\begin{aligned}
\vartheta_{g} & =-\frac{104}{171} \frac{1-\frac{2}{13} R}{1-\frac{3}{19} R} \frac{\epsilon_{1}^{2}}{1-2 R} \\
\vartheta_{y} & =\frac{4}{19} \frac{1}{1-\frac{3}{19} R} \frac{\epsilon_{1}}{1-2 R} \\
\vartheta_{u} & =\frac{16 \sqrt{23-4 R}}{19-3 R} \frac{\epsilon_{1}}{1-2 R} \\
\vartheta_{v} & =\frac{8 \sqrt{20+6 \sqrt{23-4 R}-4 R}}{19-3 R} \frac{\epsilon_{1}}{1-2 R} .
\end{aligned}
$$

For $\epsilon_{1}>0$ and $R<\frac{1}{2}$ asymptotic safety is guaranteed with $\vartheta_{g}<0<\vartheta_{y}, \vartheta_{u}, \vartheta_{v}$, showing that the UV fixed point has one relevant direction. The scaling exponents reduce to those in [32] for $R=0$. Conversely, for $\epsilon_{1}<0$ and $R>\frac{1}{2}$ the theory is asymptotically free and the interacting fixed point is fully IR attractive with $0<\vartheta_{g}, \vartheta_{y}, \vartheta_{u}, \vartheta_{v}$. Results straightforwardly translate to the partially interacting fixed points $\mathrm{FP}_{3}$ and $\mathrm{FP}_{5}$ where $\alpha_{1}^{*} \equiv 0$. The explicit $\beta$-functions in the other gauge sector are found from (63)-(66) via the replacements $\epsilon_{1} \leftrightarrow \epsilon_{2}$ and $R \leftrightarrow 1 / R$, leading to

$\beta_{2}=\frac{4}{3} \epsilon_{2} \alpha_{2}^{2}+\left(25+\frac{26}{3} \epsilon_{2}\right) \alpha_{2}^{3}-2\left(\epsilon_{2}-\frac{1}{R}+\frac{11}{2}\right)^{2} \alpha_{2}^{2} \alpha_{y}$

$\beta_{y}=\left[13+2\left(\epsilon_{2}-\frac{1}{R}\right)\right] \alpha_{y}^{2}-6 \alpha_{y} \alpha_{2}$

$\beta_{u}=-\left[11+2\left(\epsilon_{2}-\frac{1}{R}\right)\right] \alpha_{y}^{2}+4 \alpha_{u}\left(\alpha_{y}+2 \alpha_{u}\right)$,

$\beta_{v}=12 \alpha_{u}^{2}+4 \alpha_{v}\left(\alpha_{v}+4 \alpha_{u}+\alpha_{y}\right)$.

Evidently, the coordinates of the fully interacting gaugeYukawa fixed point and the corresponding universal scaling exponents of (67) are given by (65), (66) after obvious replacements. Moreover, in (67) the parameter $R$ with

$$
0 \leq \frac{1}{R}=\frac{N_{2}}{N_{\mathrm{c}}}<\frac{11}{2}
$$

measures the number of Yukawa-less Dirac fermions $N_{2}$ in the fundamental representation in units of $N_{\mathrm{c}}$, see (28). The only direct communication between the different gauge sectors in (25) is through the off-diagonal two-loop gauge contributions $G_{i}$. Were it not for the fermions $\psi$ which are charged under both gauge groups, the theory (25) with (55), (57) would be the "direct product" of the simple model (63), (64) with its counterpart (67), (68). In this limit we will find nine "direct product" fixed points with scaling exponents from each pairing of the possibilities $(\mathrm{G}, \mathrm{BZ}, \mathrm{GY})$ in each sector.

Below, we contrast findings for the full semisimple setting (55), (57) with those from the "direct product" limit in order to pin-point effects which uniquely arise from the semisimple character of the theories (25).

At any of the partially interacting fixed points, the semisimple character of the theory becomes visible in the noninteracting sector. In fact, contributions from the $\psi$ fermions modify the effective one-loop coefficient $B_{i} \rightarrow B_{i}^{\prime}$ according to

$$
\begin{aligned}
& \alpha_{1}^{*}=0: B_{1} \rightarrow B_{1}^{\prime}=B_{1}+G_{1} \alpha_{2}^{*} \\
& \alpha_{2}^{*}=0: B_{2} \rightarrow B_{2}^{\prime}=B_{2}+G_{2} \alpha_{1}^{*} .
\end{aligned}
$$

No such effects can materialize in a "direct product" limit. Moreover, these contributions always arise with a positive 
coefficient $\left(B^{\prime}>B\right)$ and are absent if $N_{\psi}=0$ (where $G_{i}=0$ ). For $N_{\psi} \neq 0$, asymptotic freedom can thereby be changed into infrared freedom, but not the other way around. This result is due to the fact that the Yukawa couplings are tied to individual gauge groups, and so by this structure we cannot have any Yukawa contributions to $B^{\prime}$. In principle, the opposite effect can equally arise: it would require Yukawa couplings which contribute to both gauge coupling $\beta$-functions, and would therefore have to involve at least one field which is charged under both gauge groups [34]. Table VI shows the effective one loop coefficients at partially interacting fixed points as a function of field multiplicities.

\section{Gauss with Banks-Zaks}

Next, we discuss all fixed points one-by-one, and determine the valid parameter regimes $(R, P, \epsilon)$ for each of them. We recall that $N_{\psi}=1$ in our models. Whenever appropriate, we also compare results with the "direct product" limit, whereby the diagonal contributions from the Yukawa-less $\psi$-fermions are retained but their off-diagonal contributions to the other gauge sectors suppressed (see Sec. IV B). This comparison allows us to quantify the effect related to the semisimple nature of the models (25).

For convenience and better visibility, we scale the axes in Figs. $1-5$ as

$$
X \rightarrow \frac{X}{1+|X|} \quad \text { where } X=P \quad \text { or } \quad R
$$

and within their respective domains of validity $R \in\left(\frac{2}{11}, \frac{11}{2}\right)$ and $P \in(-\infty, \infty)$. The rescaling permits easy display of the entire range of parameters.

Figure 1 shows the results for $\mathrm{FP}_{2}(\mathrm{BZ} \cdot \mathrm{G}$, upper) and $\mathrm{FP}_{3}(\mathrm{G} \cdot \mathrm{BZ}$, lower panel), and parameter ranges are given in Table VII. We observe that the Banks-Zaks fixed point always requires an asymptotically free gauge sector. Hence, $\mathrm{FP}_{2}$ exists for any $R$ as long as $\epsilon<0$. Provided that $P \epsilon<0$, the other gauge sector either remain asymptotically free (region 1) or becomes infrared free (region 2). On the other hand, if $P \epsilon>0$, the other gauge sector is invariably infrared free. This is a consequence of (69) which states that the interacting gauge sector can turn asymptotic freedom of the non-interacting gauge sector into infrared freedom (region 2), but not the other way around. The existence of the parameter region 2 is thus entirely due to the semisimple character of the theory which cannot arise from a "direct product."

The Banks-Zaks fixed point is invariably attractive in the gauge coupling, and repulsive in the Yukawa coupling. The eigenvalue spectrum in the gauge-Yukawa sector is therefore of the form (17) or (18), depending on whether the free gauge sector is asymptotically free or infrared free, see Table VII.
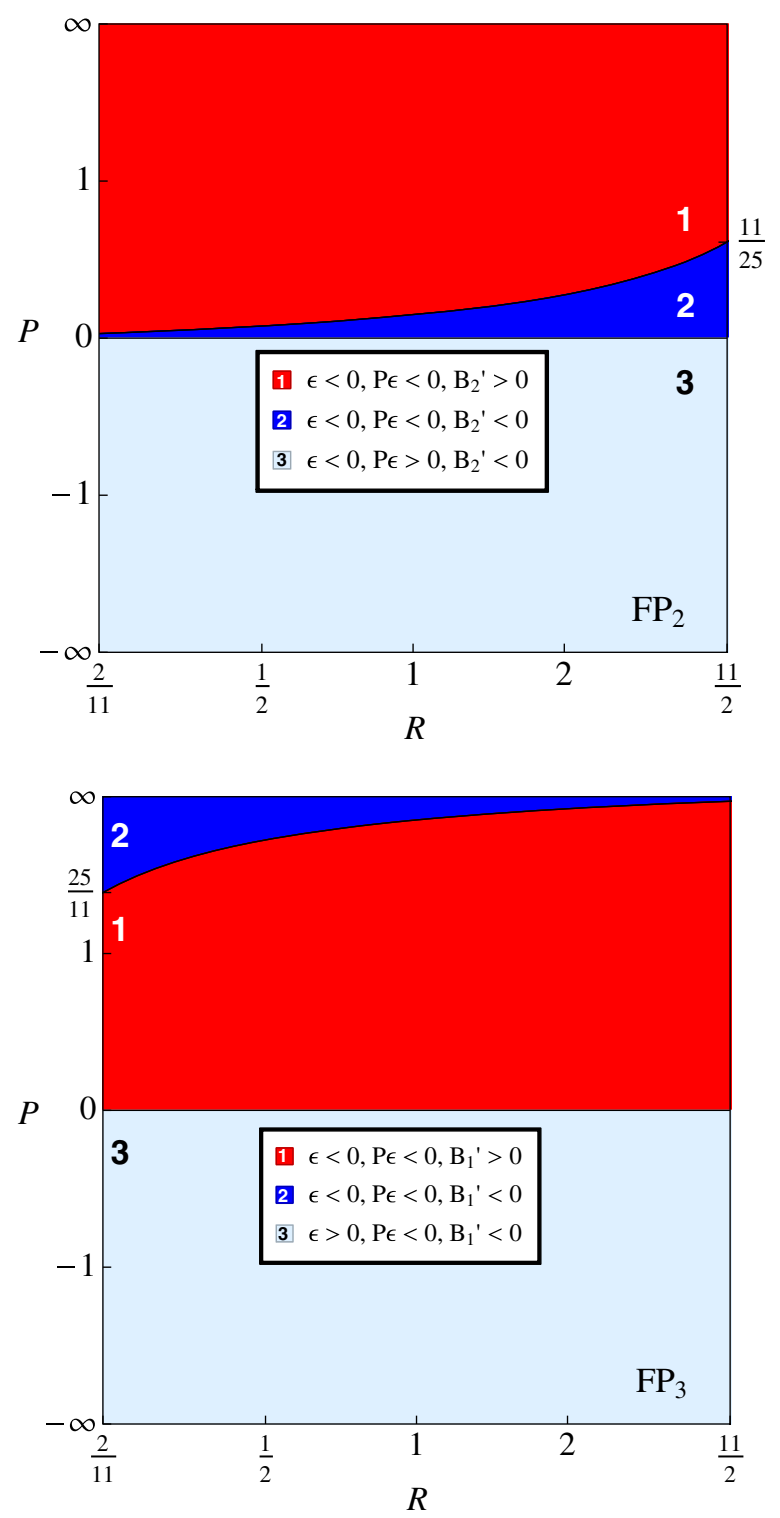

FIG. 1. The phase space of parameters (61) for the partially interacting fixed points $\mathrm{FP}_{2}$ (upper panel) and $\mathrm{FP}_{3}$ (lower panel) where one of the two gauge sectors remains interacting and all Yukawa couplings vanish. The inset indicates the different parameter regions and conditions for existence, including whether the non-interacting gauge sector is asymptotically free $\left(B^{\prime}>0\right)$ or infrared free $\left(B^{\prime}<0\right)$, see Tables VI, VII.

Under the exchange of gauge groups we have $(R, P, \epsilon) \leftrightarrow\left(R^{-1}, P^{-1}, P \epsilon\right)$, see (46). On the level of Fig. 1 this corresponds to a simple rotation by 180 degree around the symmetric points $(R, P)=(1,1)$ (for $P>0$ ) and $(R, P)=(1,-1)$ (for $P<0$ ), owing to the rescaling of parameters. Consequently, the results for $\mathrm{FP}_{3}$ can be deduced from those at $\mathrm{FP}_{2}$ by a simple rotation, see Fig. 1. More generally, this exchange symmetry relates the partially interacting fixed points $\mathrm{FP}_{2} \leftrightarrow \mathrm{FP}_{3}$ (Fig. 1), $\mathrm{FP}_{4} \leftrightarrow \mathrm{FP}_{5}$ (Fig. 2), and the fully interacting fixed points $\mathrm{FP}_{7} \leftrightarrow \mathrm{FP}_{8}$ (Fig. 4). The exchange symmetry is manifest at 
the fully interacting fixed points $\mathrm{FP}_{6}$ (Fig. 3) and $\mathrm{FP}_{9}$ (Fig. 5).

\section{Gauss with Gauge-Yukawa}

In Fig. 2 we show the domains of existence for $\mathrm{FP}_{4}$ (GY $\cdot \mathrm{G}$, upper) and $\mathrm{FP}_{5}(\mathrm{G} \cdot \mathrm{GY}$, lower panel). We observe that the fixed point exists for any parameter choice though its features vary with matter multiplicities. Specifically, for $\mathrm{FP}_{4}$, six qualitatively different parameter regions are found. If the interacting gauge coupling is asymptotically free $(\epsilon<0)$ and provided that $P \epsilon<0$, the other gauge
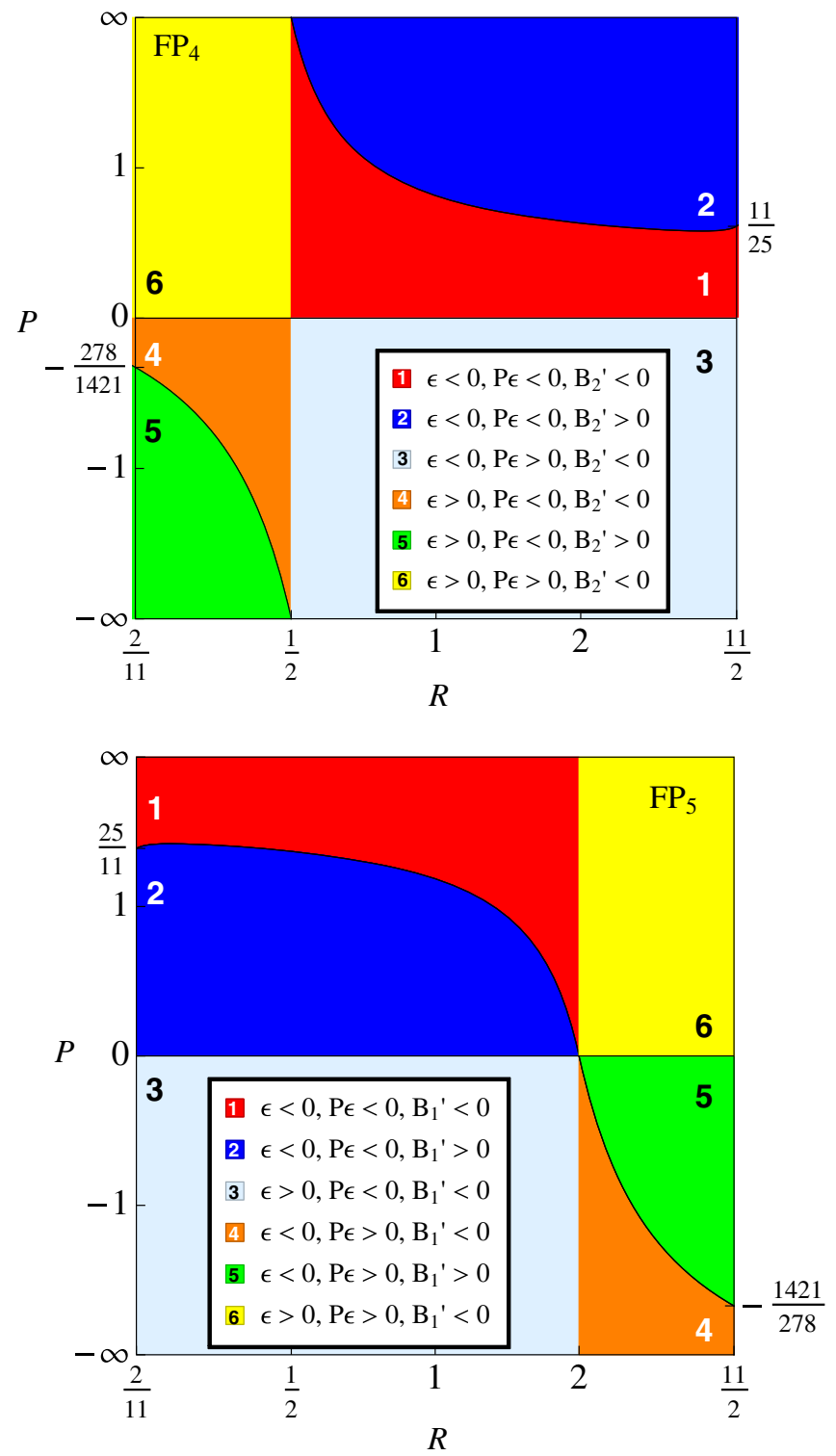

FIG. 2. The phase space of parameters for the partially interacting fixed points $\mathrm{FP}_{4}$ and $\mathrm{FP}_{5}$, where the gauge and Yukawa coupling in one gauge sector take interacting fixed points while those of the other sector remain trivial. The insets indicate the different parameter regions and conditions for existence, and whether the non-interacting gauge sector is asymptotically free $\left(B^{\prime}>0\right)$ or infrared free $\left(B^{\prime}<0\right)$, see Tables VI, VII. sector either remains asymptotically free (region 2) or becomes infrared free (region 1), whereas for $P \epsilon>0$ the other gauge sector invariably remains infrared free (region 3). Conversely, if the interacting gauge coupling is infrared free $(\epsilon>0)$ and provided that $P \epsilon<0$, the other gauge sector either remains asymptotically free (region 5 ) or becomes infrared free (region 4), whereas for $P \epsilon>0$ the other gauge sector invariably remains infrared free (region 6). Moreover, as explained in Table VI, the interacting gauge sector can turn asymptotic freedom of the noninteracting gauge sector into infrared freedom (region 1 and 4). The eigenvalue spectrum in the gauge-Yukawa sector has therefore no relevant eigendirection (20) in region 1 and 3 , one relevant eigendirection (18) in region 4 and 6 , two relevant eigendirections (19) in region 2, and three relevant eigendirections (17) in region 5, see Table VII.

We make the following observations. First, we note that $\mathrm{FP}_{4}$ in region 1 and 3 corresponds to a fully attractive IR fixed point with all $\mathrm{RG}$ trajectories terminating in it. The fixed point then acts as an infrared "sink" for massless trajectories and all canonically marginal couplings of the theory. Once scalar masses are switched on, RG flows may run away from the hypercritical surface of exactly massless theories, leading to massive phases with or without spontaneous breaking of symmetry. The quantum phase transition at $\mathrm{FP}_{4}$ in region 1 and 3 is of the second order. Notice that in the "direct product" limit only models with $P \epsilon>0>\epsilon$ and $R>\frac{1}{2}$ (analogous to region 3) would lead to a fully infrared attractive "sink." Hence, the availability of region 1 is an entirely new effect, solely due to the $\psi$ fermions and the semisimple nature of our models. We conclude that the semisimple structure opens up new types of fixed points which cannot be achieved through a product structure. In region 2, we find that $\mathrm{FP}_{4}$ has two relevant eigendirections as it would in direct product settings.

Second, in regions 4 and $6, \mathrm{FP}_{4}$ shows a single relevant eigendirection. In the direct product limit, only models with $P, \epsilon>0$ and $R<\frac{1}{2}$ (analogous to region 6) would lead to a single relevant direction. Again, the availability of region 4 is a novel feature, and solely due to the $\psi$ fermions and thus a consequence of the semisimple nature of the model.

In the parameter region 5 the fixed point shows the largest number of UV relevant directions as it would without the $\psi$ fermions. Moreover, in this parameter regime the Gaussian fixed point has only two relevant directions $(\epsilon>0, P \epsilon<0)$. Therefore $\mathrm{FP}_{4}$ in region 5 qualifies as an asymptotically safe UV fixed point. On the other hand, in region 2,4 and 6, it takes the role of a cross-over fixed point. Results for $\mathrm{FP}_{5}$ (Fig. 2, lower panel) follow from those for $\mathrm{FP}_{4}$ via (46), and the distinct regions stated for $\mathrm{FP}_{5}$ relate to the same physics as those for $\mathrm{FP}_{4}$.

\section{E. Banks-Zaks with Banks-Zaks}

Next, we turn to fully interacting fixed points where both gauge couplings are nonvanishing, see Table VIII. In 
general, the eigenvalue spectrum is determined through (22) with solutions (23), with $\epsilon$ taking the role of the parameter $B$. In the direct product limit, fully interacting fixed points reduce to direct products from each pairing of the possibilities (BZ, GY) in each of the simple gauge sectors. For $N_{\psi} \neq 0$, the fermions $\psi$ introduce a direct mixing between the gauge groups and we may then expect to find something close to a product structure, potentially modified by new effects parametrized via $R$ in fixed points not involving Gaussian factors.

The first such fixed point is $\mathrm{FP}_{6}(\mathrm{BZ} \cdot \mathrm{BZ})$, where each gauge sector achieves a Banks-Zaks fixed point. Yukawa couplings play no role, see Fig. 3. The fixed point invariably requires $\epsilon<0$ and $P \epsilon<0$ and entails an eigenvalue spectrum with two relevant directions of order $\mathcal{O}\left(\epsilon^{2}\right)$, and two irrelevant directions of order $\mathcal{O}(\epsilon)$ associated to the Yukawas,

$$
\vartheta_{1}, \vartheta_{2}<0<\vartheta_{3}, \vartheta_{4}
$$

The quartics are marginally irrelevant. The Gaussian is necessarily the UV fixed point in these settings which makes $\mathrm{FP}_{6}$ a cross-over fixed point. The accessible parameter region, shown in Fig. 3, is invariant under the exchange of gauge groups (46). The direct product limit has qualitatively the same spectrum (71). The main effect due to the semisimple character of the theory relates to the exclusion

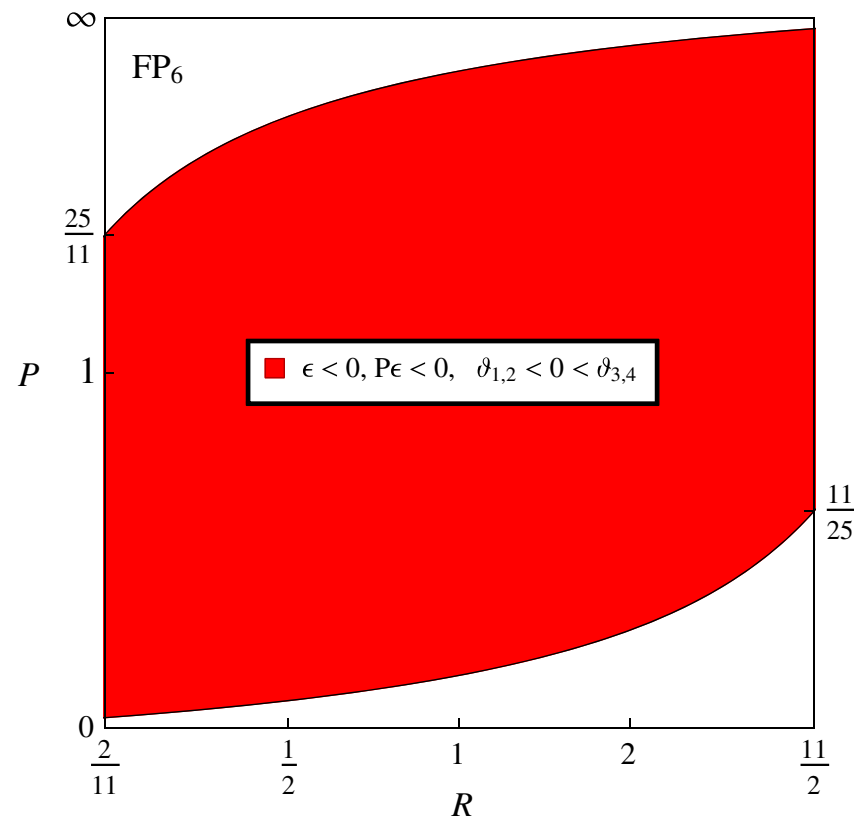

FIG. 3. The phase space of parameters for the interacting fixed point $\mathrm{FP}_{6}$ (red) where both gauge sectors take interacting and physical fixed points while all Yukawa couplings vanish. The eigenvalue spectrum at the fixed point always displays exactly two relevant eigenvalues of $O(\epsilon)$ and two irrelevant eigenvalues of order $O\left(\epsilon^{2}\right)$, see Table VIII. Note that this fixed point invariably requires asymptotic freedom for both gauge sectors (see main text). of certain parameter regions (white regions). We conclude that the semisimple nature of the theory leads to parameter restrictions without otherwise changing the overall appearance of the fixed point.

\section{F. Banks-Zaks with Gauge-Yukawa}

At the interacting fixed points $\mathrm{FP}_{7}(\mathrm{BZ} \cdot \mathrm{GY}$, upper panel), and $\mathrm{FP}_{8}(\mathrm{GY} \cdot \mathrm{BZ}$, lower panel), we have that both gauge and one of the Yukawa couplings are nontrivial. Our results for the condition of existence and the eigenvalue spectra are displayed in Fig. 4. By definition, this type of
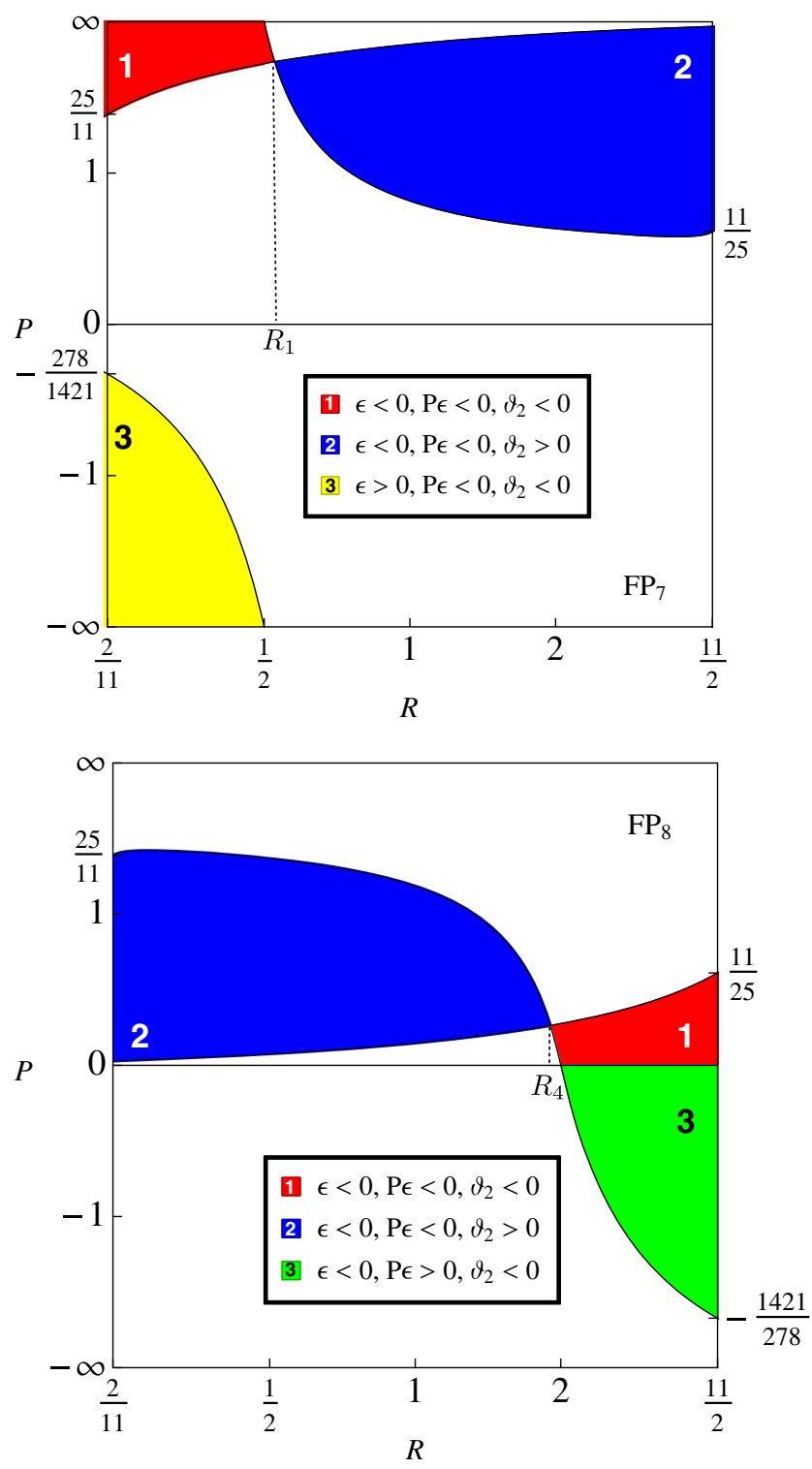

FIG. 4. The phase space of parameters for the fixed points $\mathrm{FP}_{7}$ and $\mathrm{FP}_{8}$ where two gauge and one of the Yukawa couplings take interacting and physical fixed points, while the other Yukawa coupling remains trivial. The inset indicates the signs for $\epsilon$ and $P \epsilon$, together with the sign for the eigenvalue $\vartheta_{2}$, Table VIII (see main text). 
fixed point requires that either $\epsilon<0$ or $P \epsilon<0$, or both, meaning that at least one of the gauge sectors is asymptotically free. In Fig. 4, this relates to three different parameter regions (see inset for the color coding). In region 1 and 2, the theory is asymptotically free in both gauge sectors, whereas in region 3 the theory is asymptotically free in only one gauge sector. We observe that large regions of parameter space are excluded. Valid parameter regions are further distinguished by their eigenvalue spectrum which either takes the form (19) or (18), meaning that minimally one and maximally two eigenoperators constructed out of the gauge kinetic terms and the Yukawa interactions are UV relevant, $\vartheta_{1}<0<\vartheta_{3}, \vartheta_{4}$. The sign of $\vartheta_{2}$ depends on the matter field multiplicities. In region 1 and 3, and for either of $\mathrm{FP}_{7}$ and $\mathrm{FP}_{8}$, we find that

$$
\vartheta_{1}, \vartheta_{2}<0<\vartheta_{3}, \vartheta_{4}
$$

In region 2 , conversely, we have

$$
\vartheta_{1}<0<\vartheta_{2}, \vartheta_{3}, \vartheta_{4} .
$$

Hence, at $\mathrm{FP}_{7}$ and in the regime where both gauge sectors are asymptotically free $(P>0>\epsilon)$, two types of valid fixed points are found. For sufficiently low $R<R_{1}$ (62), and large $P$, the fixed point has two relevant directions (region 1). Increasing $R>R_{1}$ at fixed $P$ may lead to a second type of IR fixed point with a single relevant direction (region 2). On the other hand, in the regime $\epsilon>0>P$ only one type of fixed point exists with two relevant directions (region 3). It is worth comparing these results with the direct product limit. For $P>0>\epsilon$ the latter leads to the eigenvalue spectrum (73), as found in region 2. Also, for $\epsilon>0>P$ the direct product fixed point has the eigenvalue spectrum (72), which is qualitatively in accord with findings in region 3 . We conclude that the semisimple nature of the interactions plays a minor quantitative role in region 2 and 3. On the other hand, in region 1 where $P>0>\epsilon$, the semisimple nature of the theory leads to an important qualitative modification: an eigenvalue spectrum with two relevant directions at $\mathrm{FP}_{7}$ cannot be achieved through a direct product setting; rather, it necessarily requires matter fields charged under both gauge groups. We conclude that the semisimple nature of interactions play a key qualitative role in region 1 . Analogous results hold true for $\mathrm{FP}_{8}$ after the substitutions (46) and the replacement $R_{1} \rightarrow R_{4}=1 / R_{1}$, see (62).

\section{G. Gauge-Yukawa with gauge-Yukawa}

At the fully interacting fixed point $\mathrm{FP}_{9}(\mathrm{GY} \cdot \mathrm{GY})$, we have that both gauge and both Yukawa couplings are nontrivial. We find that the eigenvalue spectrum in the gauge-Yukawa sector reads either (18) or (20), meaning that at least three of the four eigenoperators constructed out of the gauge and fermion fields are strictly irrelevant,

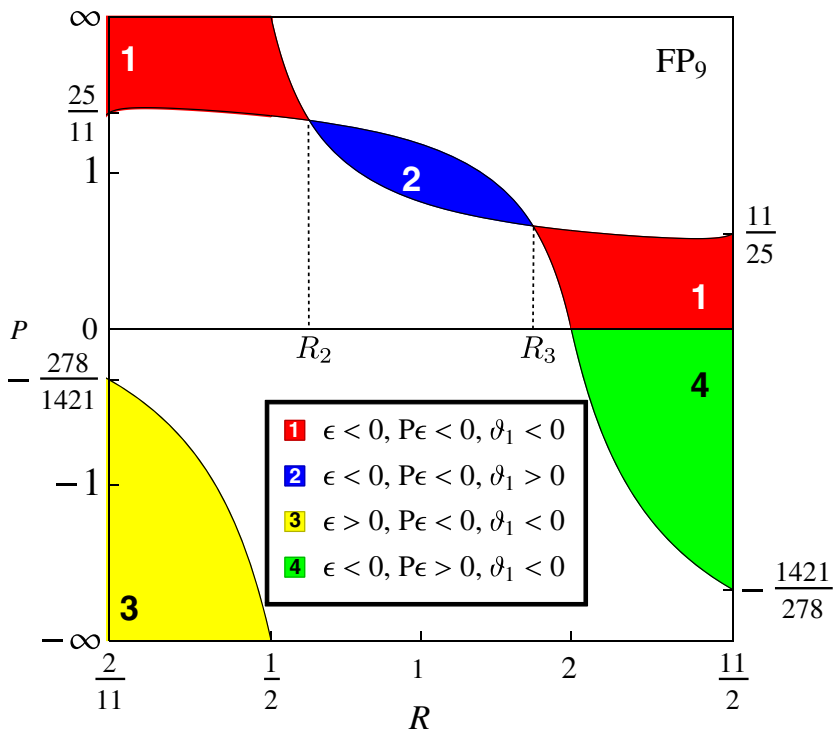

FIG. 5. The phase space of parameters for the fully interacting fixed point $\mathrm{FP}_{9}$ where all gauge and all Yukawa couplings are nontrivial. The coloured regions relate to the portions of parameter space where the fully interacting fixed point is physical. The inset provides additional information including the sign for the eigenvalue $\vartheta_{4}$ (see main text).

$0<\vartheta_{2}, \vartheta_{3}, \vartheta_{4}$. The sign of the eigenvalue $\vartheta_{1}$ depends on the matter field multiplicities of the model.

Our results for the condition of existence and the eigenvalue spectrum are stated in Fig. 5. We observe four qualitatively different parameter regions (see inset for the color coding). For $P>0>\epsilon$, the theory is asymptotically free in both gauge sectors and we find two types of valid parameter regions, depending on whether $R$ takes values below $R_{2}$ or above $R_{3}$ (region 1), or in between (region 2); see (62). Moreover, in region 2, we find that the fixed point is strictly IR attractive in all couplings, owing to

$$
0<\vartheta_{1}, \vartheta_{2}, \vartheta_{3}, \vartheta_{4} \text {. }
$$

Hence, the fixed point $\mathrm{FP}_{9}$ in region 2 corresponds to a fully attractive IR fixed point acting as an infrared sink for massless trajectories and all canonically marginal couplings of the theory. Ultimately it describes a second order quantum phase transition between a symmetric and a symmetry broken phase, characterized by the vacuum expectation value of the scalar field. Qualitatively, the same type of result is achieved in the direct product limit. Hence, the main effect due to semisimple interactions is to have generated a boundary in parameter space. In region 1 we find

$$
\vartheta_{1}<0<\vartheta_{2}, \vartheta_{3}, \vartheta_{4} .
$$

This type of eigenvalue spectrum cannot be achieved without semisimple interactions mediated by the $\psi$ fields 
TABLE VI. Shown are the effective one-loop coefficients $B^{\prime}$ for the noninteracting gauge coupling at $\mathrm{FP}_{2}, \mathrm{FP}_{3}, \mathrm{FP}_{4}$, and $\mathrm{FP}_{5}$, and their dependence on model parameters. $B^{\prime}>0$ corresponds to asymptotic freedom. Notice that $B^{\prime}$ changes sign across the boundaries $P=2 R / 25,25 R / 2, X(R)$, and $\tilde{X}(R)$, respectively, with $X$ and $\tilde{X}$ given in (B1).

\begin{tabular}{lc}
\hline \hline Fixed point & $B^{\prime}$ Coefficient \\
\hline $\mathbf{F P}_{\mathbf{2}}$ & $B_{2}^{\prime}=-\frac{4}{3}\left(1-\frac{2}{25} R / P\right) \frac{P \epsilon}{R}$ \\
$\mathbf{F P}_{\mathbf{3}}$ & $B_{1}^{\prime}=-\frac{4}{3}\left(1-\frac{2}{25} P / R\right) R \epsilon$ \\
$\mathbf{F P}_{4}$ & $B_{2}^{\prime}=-\frac{4}{3}(1-X(R) / P) \frac{P \epsilon}{R}$ \\
$\mathbf{F P}_{\mathbf{5}}$ & $B_{1}^{\prime}=-\frac{4}{3}(1-P / \tilde{X}(R)) R \epsilon$ \\
\hline \hline
\end{tabular}

and is therefore a novel feature, entirely due to the semisimple nature of the theory. In this regime, $\mathrm{FP}_{9}$ corresponds to a crossover fixed point (the Gaussian is the UV fixed point) with a single unstable direction where trajectories escape either towards a weakly coupled IR fixed point, or towards a regime of strong coupling with (chiral) symmetry breaking, confinement, or infrared conformality.

For $\epsilon>0>P$ or $P \epsilon>0>\epsilon$, the theory is asymptotically free in one and infrared free in the other gauge sector.
Valid fixed points then correspond to region 3 or region 4, respectively. In either of these cases, the eigenvalue spectrum shows a single relevant direction, (75). This agrees qualitatively with the eigenvalue spectrum in the direct product limit. We conclude, once more, that the main impact of the $\psi$ fields relates to the boundaries in parameter space which restrict the fixed point's domain of availability.

Finally, for $\epsilon, P>0$, the theory is infrared free in both gauge sectors. We observe that no such interacting fixed point arises, irrespective of matter multiplicities. Interestingly though, such fixed points do exist in the direct product limit with spectrum

$$
\vartheta_{1}, \vartheta_{2}<0<\vartheta_{3}, \vartheta_{4} \text {. }
$$

The reason for their nonexistence in our models is the presence of the $\psi$ fermions. The requirement of perturbativity in both gauge couplings then leads to limitations on the parameter $R$ which cannot be satisfied at $\mathrm{FP}_{9}$ with eigenvalue spectrum (76). This result provides us with an example where the semisimple nature of the theory "disables" a fixed point. This completes the overview of

TABLE VII. Parameter regions where the partially interacting fixed points $\mathrm{FP}_{1}-\mathrm{FP}_{5}$ exist, along with regions of relevancy for eigenvalues and effective one-loop terms, where applicable. The boundary functions $X(R)$ and $\tilde{X}(R)$ are given in (B1). The coefficient $B^{\prime}$ for the gauge coupling at the Gaussian fixed point is given in Table VI.

\begin{tabular}{|c|c|c|c|c|c|}
\hline \multirow[b]{2}{*}{ Fixed point } & \multicolumn{3}{|c|}{ Parameter range } & \multirow[b]{2}{*}{ Eigenvalue spectrum } & \multirow[b]{2}{*}{ Info } \\
\hline & $\operatorname{sign} \epsilon$ & $R$ & $P$ & & \\
\hline $\mathbf{F P}_{1}$ & \pm & $\left(\frac{2}{11}, \frac{11}{2}\right)$ & $(-\infty,+\infty)$ & $(16),(19)$, or $(20)$ & Gaussian \\
\hline \multirow[t]{4}{*}{$\mathbf{F P}_{2}$} & & & & & Fig. 1 (upper panel) \\
\hline & - & $\left(\frac{2}{11}, \frac{11}{2}\right)$ & $\left(\frac{2}{25} R,+\infty\right)$ & $\vartheta_{1,2,3} \leq 0<\vartheta_{4}$ & region 1 \\
\hline & - & $\left(\frac{2}{11}, \frac{11}{2}\right)$ & $\left(0, \frac{2}{25} R\right)$ & $\vartheta_{1}<0 \leq \vartheta_{2,3,4}$ & region 2 \\
\hline & - & $\left(\frac{2}{11}, \frac{11}{2}\right)$ & $(-\infty, 0)$ & $\vartheta_{1}<0 \leq \vartheta_{2,3,4}$ & region 3 \\
\hline \multirow[t]{4}{*}{$\mathbf{F P}_{3}$} & & & & & Fig. 1 (lower panel) \\
\hline & - & $\left(\frac{2}{11}, \frac{11}{2}\right)$ & $\left(0, \frac{25}{2} R\right)$ & $\vartheta_{1,2,3} \leq 0<\vartheta_{4}$ & region 1 \\
\hline & - & $\left(\frac{2}{11}, \frac{11}{2}\right)$ & $\left(\frac{25}{2} R,+\infty\right)$ & $\vartheta_{1}<0 \leq \vartheta_{2,3,4}$ & region 2 \\
\hline & + & $\left(\frac{2}{11}, \frac{11}{2}\right)$ & $(-\infty, 0)$ & $\vartheta_{1}<0 \leq \vartheta_{2,3,4}$ & region 3 \\
\hline \multirow[t]{5}{*}{$\mathbf{F P}_{4}$} & & & & & Fig. 2 (upper panel) \\
\hline & - & $\left(\frac{1}{2}, \frac{11}{2}\right)$ & $(-\infty, X(R))$ & $0 \leq \vartheta_{1,2,3,4}$ & region $1 \& 3$ \\
\hline & - & $\left(\frac{1}{2}, \frac{11}{2}\right)$ & $(X(R),+\infty)$ & $\vartheta_{1,2} \leq 0<\vartheta_{3,4}$ & region 2 \\
\hline & + & $\left(\frac{2}{11}, \frac{1}{2}\right)$ & $(X(R),+\infty)$ & $\vartheta_{1}<0 \leq \vartheta_{2,3,4}$ & region $4 \& 6$ \\
\hline & + & $\left(\frac{2}{11}, \frac{1}{2}\right)$ & $(-\infty, X(R))$ & $\vartheta_{1,2,3} \leq 0<\vartheta_{4}$ & region 5 \\
\hline \multirow[t]{7}{*}{$\mathbf{F P}_{5}$} & & & & & Fig. 2 (lower panel) \\
\hline & - & $\left(\frac{2}{11}, 2\right)$ & $(\tilde{X}(R),+\infty)$ & $0 \leq \vartheta_{1,2,3,4}$ & region 1 \\
\hline & - & $\left(\frac{2}{11}, 2\right)$ & $(0, \tilde{X}(R))$ & $\vartheta_{1,2} \leq 0<\vartheta_{3,4}$ & region 2 \\
\hline & + & $\left(\frac{2}{11}, 2\right)$ & $(-\infty, 0)$ & $0 \leq \vartheta_{1,2,3,4}$ & region 3 \\
\hline & - & $\left(2, \frac{11}{2}\right)$ & $(-\infty, \tilde{X}(R))$ & $\vartheta_{1}<0 \leq \vartheta_{2,3,4}$ & region 4 \\
\hline & - & $\left(2, \frac{11}{2}\right)$ & $(\tilde{X}(R), 0)$ & $\vartheta_{1,2,3} \leq 0<\vartheta_{4}$ & region 5 \\
\hline & + & $\left(2, \frac{11}{2}\right)$ & $(0,+\infty)$ & $\vartheta_{1}<0 \leq \vartheta_{2,3,4}$ & region 6 \\
\hline
\end{tabular}


TABLE VIII. Parameter regions where the fully interacting fixed points $\mathrm{FP}_{6}-\mathrm{FP}_{9}$ exist, along with the eigenvalue spectrum for the various parameter regions.

\begin{tabular}{|c|c|c|c|c|c|}
\hline \multirow[b]{2}{*}{ Fixed point } & \multicolumn{3}{|c|}{ Parameter range } & \multirow[b]{2}{*}{ Eigenvalue spectrum } & \multirow[b]{2}{*}{ Info } \\
\hline & Sign $\epsilon$ & $R$ & $P$ & & \\
\hline $\mathbf{F P}_{6}$ & - & $\left(\frac{2}{11}, \frac{11}{2}\right)$ & $\left(\frac{2}{25} R, \frac{25}{2} R\right)$ & $\vartheta_{1,2}<0<\vartheta_{3,4}$ & Fig. 3 \\
\hline $\mathbf{F P}_{7}$ & $\begin{array}{l}- \\
- \\
- \\
+\end{array}$ & $\begin{array}{l}\left(\frac{2}{11}, \frac{1}{2}\right) \\
\left(\frac{1}{2}, R_{1}\right) \\
\left(R_{1}, \frac{11}{2}\right) \\
\left(\frac{2}{11}, \frac{1}{2}\right)\end{array}$ & $\begin{array}{c}\left(\frac{25}{2} R,+\infty\right) \\
\left(\frac{25}{2} R, X(R)\right) \\
\left(X(R), \frac{25}{2} R\right) \\
(-\infty, X(R))\end{array}$ & $\begin{array}{l}\vartheta_{1,2}<0<\vartheta_{3,4} \\
\vartheta_{1,2}<0<\vartheta_{3,4} \\
\vartheta_{1}<0<\vartheta_{2,3,4} \\
\vartheta_{1,2}<0<\vartheta_{3,4}\end{array}$ & $\begin{array}{c}\text { Fig. } 4 \text { (upper panel) } \\
\text { region } 1 \\
\text { region } 1 \\
\text { region } 2 \\
\text { region } 3\end{array}$ \\
\hline $\mathbf{F P}_{8}$ & - & $\begin{array}{l}\left(R_{4}, \frac{11}{2}\right) \\
\left(\frac{2}{11}, R_{4}\right)\end{array}$ & $\begin{array}{l}\left(\tilde{X}(R), \frac{2}{25} R\right) \\
\left(\frac{2}{25} R, \tilde{X}(R)\right)\end{array}$ & $\begin{array}{l}\vartheta_{1,2}<0<\vartheta_{3,4} \\
\vartheta_{1}<0<\vartheta_{2,3,4}\end{array}$ & $\begin{array}{c}\text { Fig. } 4 \text { (lower panel) } \\
\text { region } 1 \& 3 \\
\text { region } 2\end{array}$ \\
\hline $\mathbf{F P}_{\mathbf{9}}$ & $\begin{array}{l}- \\
- \\
- \\
- \\
+\end{array}$ & $\begin{array}{l}\left(\frac{2}{11}, \frac{1}{2}\right) \\
\left(\frac{1}{2}, R_{2}\right) \\
\left(R_{3}, \frac{11}{2}\right) \\
\left(R_{2}, R_{3}\right) \\
\left(\frac{2}{11}, \frac{1}{2}\right)\end{array}$ & $\begin{array}{c}(\tilde{X}(R),+\infty) \\
(\tilde{X}(R), X(R)) \\
(\tilde{X}(R), X(R)) \\
(X(R), \tilde{X}(R)) \\
(-\infty, X(R))\end{array}$ & $\begin{array}{c}\vartheta_{1}<0<\vartheta_{2,3,4} \\
\vartheta_{1}<0<\vartheta_{2,3,4} \\
\vartheta_{1}<0<\vartheta_{2,3,4} \\
0<\vartheta_{1,2,3,4} \\
\vartheta_{1}<0<\vartheta_{2,3,4}\end{array}$ & $\begin{array}{l}\text { Fig. } 5 \\
\text { region } 1 \\
\text { region } 1 \\
\text { region } 1 \& 4 \\
\text { region } 2 \\
\text { region } 3\end{array}$ \\
\hline
\end{tabular}

interacting fixed points in the gauge-Yukawa sector and their key properties.

\section{SCALAR FIXED POINTS AND VACUUM STABILITY}

In this section, we analyse the scalar sector and establish conditions for stability of the quantum vacuum. We also provide results for all scalar couplings at all interacting fixed points, Table IX.

\section{A. Yukawa and scalar nullclines}

Following [34], we begin by exploiting the results (53) to express the Yukawa nullclines in terms of the gauge couplings and the parameter $R$. To leading order in the small expansion parameters (38), and using (53) together with (10), the nontrivial Yukawa nullclines $\beta_{Y}=0$ and $\beta_{y}=0$ take the explicit form

$$
\frac{\alpha_{Y}}{\alpha_{1}}=\frac{6}{13-2 R}, \quad \frac{\alpha_{y}}{\alpha_{2}}=\frac{6}{13-2 / R} .
$$

For fixed gauge couplings, we observe that the Yukawa couplings are enhanced over their values in the absence of the fermions $\psi$. The relevance of nullcline solutions (77) is as follows. By their very definition, the Yukawa couplings no longer run with the RG scale when taking the values (77). If at the same time the gauge couplings take fixed points on their own, the nullcline relations then provide us with the correct fixed point values for the Yukawa couplings. Evidently, (77) together with (39) guarantees that the Yukawa fixed points are physical as long as the gauge fixed points are. Note also that the slope of the nullcline remains positive and finite for all $R$ within the domain (39). Hence strict perturbativity in the Yukawa couplings follows from strict perturbativity in the gauge couplings, in accord with the general discussion in [34] based on dimensional analysis.

Next we turn to the scalar nullclines. Since the beta functions for the two scalar sectors decouple at this order, we may analyse their nullclines individually. ${ }^{4}$ All results for the subsystem $\left(\alpha_{U}, \alpha_{V}\right)$ can straightforwardly be translated to the subsystem $\left(\alpha_{u}, \alpha_{v}\right)$ by substituting $R \leftrightarrow 1 / R$, also using (38). Furthermore, since the scalars are uncharged, their one loop beta functions are independent of the gauge coupling. Dimensional analysis then shows that all nontrivial scalar nullclines are proportional to the corresponding Yukawa coupling [34]. The scalar nullclines represent exact fixed points of the theory provided the Yukawa couplings take interacting fixed points. Perturbativity of scalar couplings at an interacting fixed point then follows from the perturbativity of Yukawa couplings which, in turn, follows from perturbativity in the gauge couplings.

Specifically, the nullclines for the single trace scalar couplings are found from (57) by resolving $\beta_{U}=0$ for $\alpha_{U}$. We find two solutions

$$
\frac{\alpha_{U \pm}}{\alpha_{Y}}=\frac{1}{4}(-1 \pm \sqrt{23-4 R}) .
$$

\footnotetext{
${ }^{4}$ This simplification solely arises provided the mixing coupling $\alpha_{w}$ takes its exact Gaussian fixed point (58). For nontrivial $\alpha_{w}$ the nullclines take more general forms.
} 
TABLE IX. Quartic scalar couplings at all weakly interacting fixed points to leading order in $\epsilon$ following Table III using the auxiliary functions (92). Same conventions as in Table V. Within the admissible parameter ranges (Tables VII, VIII) we observe vacuum stability.

\begin{tabular}{lc}
\hline \hline Fixed point & Quartic scalar couplings \\
\hline $\mathbf{F P}_{1-3}$ & $\alpha_{U}^{*}=0, \alpha_{V}^{*}=0, \alpha_{u}^{*}=0, \alpha_{v}^{*}=0$, \\
$\mathbf{F P}_{\mathbf{4}}$ & $\alpha_{U}^{*}=\frac{4 F_{1}(R) R \epsilon}{(2 R-1)(3 R-19)}, \alpha_{V}^{*}=\frac{4 F_{2}(R) R \epsilon}{(2 R-1)(3 R-19)}, \alpha_{u}^{*}=0, \alpha_{v}^{*}=0$, \\
$\mathbf{F P}_{\mathbf{5}}$ & $\alpha_{U}^{*}=0, \alpha_{V}^{*}=0, \alpha_{u}^{*}=\frac{4 F_{1}(1 / R) P \epsilon / R}{(2 / R-1)(3 / R-19)}, \alpha_{v}^{*}=\frac{4 F_{2}(1 / R) P \epsilon / R}{(2 / R-1)(3 / R-19),}$, \\
$\mathbf{F P}_{\mathbf{6}}$ & $\alpha_{U}^{*}=0, \alpha_{V}^{*}=0, \alpha_{u}^{*}=0, \alpha_{v}^{*}=0$, \\
$\mathbf{F P}_{\mathbf{7}}$ & $\alpha_{U}^{*}=\frac{4}{3} \frac{(25-2 P / R) F_{1}(R)}{50 R^{2}-343 R+167} \operatorname{Re}, \alpha_{V}^{*}=\frac{4}{3} \frac{(25-2 P / R) F_{2}(R)}{50 R^{2}-343 R+167} R \epsilon, \alpha_{u}^{*}=0, \alpha_{v}^{*}=0$, \\
$\mathbf{F P}_{\mathbf{8}}$ & $\alpha_{U}^{*}=0, \alpha_{V}^{*}=0, \alpha_{u}^{*}=\frac{4}{3} \frac{\left(25-2 R / R^{2}-343 / R+167\right.}{50 / F_{1}(1 / R)}, \alpha_{v}^{*}=\frac{4}{3} \frac{(25-2 R / P) F_{2}(1 / R)}{50 / R^{2}-343 / R+167} \frac{P \epsilon}{R}$, \\
$\mathbf{F P}_{9}$ & $\alpha_{U}^{*}=\frac{4}{3} \frac{[(13-2 / R) P / R+(2 / R-1)(3 / R-19)] F_{1}(R)}{\left(19 R^{2}-43 R+19\right)\left(2 / R^{2}-13 / R+2\right)} R \epsilon, \alpha_{u}^{*}=\frac{4}{3} \frac{[(13-2 R) R / P+(2 R-1)(3 R-19)] F_{1}(1 / R)}{\left(19 / R^{2}-43 / R+19\right)\left(2 R^{2}-13 R+2\right)} \frac{P \epsilon}{R}$, \\
& $\alpha_{V}^{*}=\frac{4}{3} \frac{[(13-2 / R) P / R+(2 / R-1)(3 / R-19)] F_{2}(R)}{\left(19 R^{2}-43 R+19\right)\left(2 / R^{2}-13 / R+2\right)} R \epsilon, \alpha_{v}^{*}=\frac{4}{3} \frac{[(13-2 R) R / P+(2 R-1)(3 R-19)] F_{2}(1 / R)}{\left(19 / R^{2}-43 / R+19\right)\left(2 R^{2}-13 R+2\right)} \frac{P \epsilon}{R}$. \\
\hline \hline
\end{tabular}

Note that the double trace coupling does not couple back into the running of the single trace coupling. Within the parameter range (39) we observe that $\alpha_{U+}>0>\alpha_{U-}$. Next, we consider the nullclines for the double-trace quartic coupling $\alpha_{V}$. Inserting $\alpha_{U+}$ into $\beta_{V}=0$, we find a pair of nullclines given by

$$
\frac{\alpha_{V \pm}}{\alpha_{Y}}=\frac{1}{4}(-2 \sqrt{23-4 R} \pm \sqrt{20-4 R+6 \sqrt{23-4 R}})
$$

Both nullclines take real values for all $R$ within the range (39), and we end up with $\alpha_{U+} \geq 0$ together with $0>\alpha_{V+}>\alpha_{V-}$. Analogously, inserting $\alpha_{U-}$ into $\beta_{V}=0$, we find a second pair of nullclines given by

$$
\frac{\alpha_{V 2 \pm}}{\alpha_{Y}}=\frac{1}{4}(2 \sqrt{23-4 R} \pm \sqrt{20-4 R-6 \sqrt{23-4 R}})
$$

In this case, however, the result (80) comes out complex within the parameter range (39), meaning that even if $\alpha_{Y}^{*}$ takes a real positive fixed point the corresponding scalar fixed point is invariably unphysical.

The replacement $R \rightarrow 1 / R$ in (78) and (79), (80) allows us to obtain explicit expressions for the nullclines for $\alpha_{u \pm} / \alpha_{y}$ and $\alpha_{v \pm} / \alpha_{y}$. The real solutions are given by

$$
\frac{\alpha_{u \pm}}{\alpha_{y}}=\frac{1}{4}(-1 \pm \sqrt{23-4 / R})
$$

with $\alpha_{u+} \geq 0>\alpha_{u-}$. The solution $\alpha_{u+}$ leads to real nullclines for the double-trace coupling $\alpha_{v}$ given by

$$
\frac{\alpha_{v \pm}}{\alpha_{y}}=\frac{1}{4}(-2 \sqrt{23-4 / R} \pm \sqrt{20-4 / R+6 \sqrt{23-4 / R}})
$$

and we end up with $\alpha_{u+} \geq 0$ together with $0>\alpha_{v+}>\alpha_{v-}$. On the other hand, the solution $\alpha_{u-}$ does not lead to real solutions for $\alpha_{v \pm}$. This completes the overview of Yukawa and scalar nullcline solutions.

\section{B. Stability of the vacuum}

We are now in a position to reach firm conclusions concerning the stability of the ground state at interacting fixed points. The reason for this is that this information is encoded in the scalar nullclines. The explicit form of the fixed point in the gauge-Yukawa sector is not needed. To that end, we recall the stability analysis for potentials of the form

$$
W \propto \alpha_{U} \operatorname{Tr}\left(H^{\dagger} H\right)^{2}+\alpha_{V} / N_{\mathrm{F}}\left(\operatorname{Tr} H^{\dagger} H\right)^{2},
$$

In the limit where $\alpha_{w}=0$ the scalar field potential in our models (25) are given by (83) together with its counterpart $\left(H, \alpha_{U}, \alpha_{V}\right) \leftrightarrow\left(h, \alpha_{u}, \alpha_{v}\right)$. For potentials of the form (83), the general conditions for vacuum stability read $[48,51]$

$$
\begin{array}{lll}
\text { a) } \alpha_{U}^{*}>0 & \text { and } & \alpha_{U}^{*}+\alpha_{V}^{*}>0 \\
\text { b) } \alpha_{U}^{*}<0 & \text { and } & \alpha_{U}^{*}+\alpha_{V}^{*} / N_{\mathrm{F}}>0
\end{array}
$$

and similarly for $\left(\alpha_{U}, \alpha_{V}\right) \leftrightarrow\left(\alpha_{u}, \alpha_{v}\right)$. In the Veneziano limit, case (b) effectively becomes void and cannot be satisfied for any $\alpha_{U}^{*}$, irrespective of the sign of $\alpha_{V}^{*}$. Inserting the fixed points into (84) we find 


$$
\begin{aligned}
\alpha_{U+}^{*}+\alpha_{V+}^{*}= & \frac{\alpha_{Y}^{*}}{4}(+\sqrt{20-4 R+6 \sqrt{23-4 R}} \\
& -\sqrt{23-4 R}-1) \geq 0, \\
\alpha_{U+}^{*}+\alpha_{V-}^{*}= & \frac{\alpha_{Y}^{*}}{4}(-\sqrt{20-4 R+6 \sqrt{23-4 R}} \\
& -\sqrt{23-4 R}-1) \leq-\alpha_{Y}^{*},
\end{aligned}
$$

Stability of the quantum vacuum is evidently achieved at the fixed point $\left(\alpha_{U+}^{*}, \alpha_{V+}^{*}\right)$ following case (a) and irrespective of the value for the Yukawa fixed point as long as $\alpha_{Y}^{*}>0$. The potential (83) becomes exactly flat at the fixed point iff $R=\frac{11}{2}$. In this case, higher order or radiative corrections must be taken into consideration to guarantee stability in the presence of flat directions. Stability is not achieved at the fixed point $\left(\alpha_{U+}^{*}, \alpha_{V-}^{*}\right)$, for any $R$. Turning to the second scalar sector, we find

$$
\begin{aligned}
\alpha_{u+}^{*}+\alpha_{v+}^{*}= & \frac{\alpha_{y}^{*}}{4}(+\sqrt{20-4 / R+6 \sqrt{23-4 / R}} \\
& -\sqrt{23-4 / R}-1) \geq 0, \\
\alpha_{u+}^{*}+\alpha_{v-}^{*}= & \frac{\alpha_{y}^{*}}{4}(-\sqrt{20-4 / R+6 \sqrt{23-4 / R}} \\
& -\sqrt{23-4 / R}-1) \leq-\alpha_{y}^{*},
\end{aligned}
$$

where the bounds refer to $R$ varying within the range (39). This part of the potential becomes exactly flat at the fixed point iff $R=\frac{2}{11}$. The result establishes vacuum stability at the fixed point $\left(\alpha_{u+}^{*}, \alpha_{v+}^{*}\right)$. We also confirm that the fixed point $\left(\alpha_{u+}^{*}, \alpha_{v-}^{*}\right)$ does not lead to a stable ground state. We conclude that vacuum stability is guaranteed at the interacting fixed points $\left(\alpha_{U+}^{*}, \alpha_{V+}^{*}\right)$ and $\left(\alpha_{u+}^{*}, \alpha_{v+}^{*}\right)$, together with $\alpha_{w}=0$, irrespective of the fixed points in the gauge Yukawa sector, as long as the later is physical. Out of the a priori $2^{3}$ different fixed point candidates in the scalar sector at one loop (half of which lead to real fixed points) the additional requirement of vacuum stability has identified a unique viable solution. In this light, vacuum stability dictates that the anomalous dimensions (49) are strictly positive at interacting fixed points, (60), with

$$
\begin{aligned}
& \gamma_{M}^{*}=\alpha_{Y}^{*} \sqrt{20-4 R+6 \sqrt{23-4 R}}>0, \\
& \gamma_{m}^{*}=\alpha_{y}^{*} \sqrt{20-4 / R+6 \sqrt{23-4 / R}}>0,
\end{aligned}
$$

and provided that (39) is observed.

\section{Portal coupling}

Now we clarify whether the stability of the vacuum is affected by the presence of the "portal" coupling $\alpha_{w} \neq 0$ which induces a mixing between the scalar sectors. In this case the scalar potential is given by $W=-L_{\text {pot }}$ in (25),

$$
\begin{aligned}
W= & U \operatorname{Tr}\left(H^{\dagger} H\right)^{2}+V\left(\operatorname{Tr} H^{\dagger} H\right)^{2}+u \operatorname{Tr}\left(h^{\dagger} h\right)^{2} \\
& +v\left(\operatorname{Tr} h^{\dagger} h\right)^{2}+w \operatorname{Tr}\left(H^{\dagger} H\right) \operatorname{Tr}\left(h^{\dagger} h\right),
\end{aligned}
$$

where $H$ and $h$ are $N_{\mathrm{F}} \times N_{\mathrm{F}}$ and $N_{\mathrm{f}} \times N_{\mathrm{f}}$ matrices, respectively. Following the reasoning of [48,51], we observe that the potential has a global $U\left(N_{\mathrm{F}}\right)_{L} \otimes U\left(N_{\mathrm{F}}\right)_{R} \otimes$ $U\left(N_{\mathrm{f}}\right)_{L} \otimes U\left(N_{\mathrm{f}}\right)_{R}$ symmetry which allows us to bring each of the fields into a real diagonal configuration, $H=$ $\operatorname{diag}\left(\Phi_{1}, \Phi_{2}, \ldots\right)$ and $h=\operatorname{diag}\left(\phi_{1}, \phi_{2}, \ldots\right)$. As the potential is homogeneous in either field, $W(c H, c h)=c^{4} W(H, h)$, it suffices to guarantee positivity on a fixed surface $\sum_{i} \Phi_{i}^{2}=$ $1=\sum_{j} \phi_{j}^{2}$ which is implemented using Lagrange multipliers $\Lambda$ and $\lambda$. From

$$
\begin{aligned}
& \frac{\partial W}{\partial \Phi_{i}}=2 \Phi_{i}\left(2 U \Phi_{i}^{2}+2 V+w-2 \Lambda\right), \\
& \frac{\partial W}{\partial \phi_{i}}=2 \phi_{i}\left(2 u \phi_{i}^{2}+2 v+w-2 \lambda\right),
\end{aligned}
$$

it follows that extremal field configurations are those where all non-zero fields take equal values. If we have $M$ nonzero $\Phi$ fields and $m$ nonzero $\phi$ fields, the extremal field values are $\Phi_{i}^{2}=0$ or $\Phi_{i}^{2}=\frac{1}{M}$ alongside with $\phi_{i}^{2}=0$ or $\phi_{i}^{2}=\frac{1}{m}$. Three nontrivial cases arise. If $m=0$ the extremal potential is $W_{e}=U / M+V$. Likewise if $M=0$ we have $W_{e}=u / m+v$. Lastly, if both $m, M \neq 0$, we have $W_{e}=U / M+V+u / m+v+w$. The values of $M, m$ for which these extremal potentials are minima depend on the signs of the couplings $U, u$, leaving us with the four possible cases $U, u>0, u>0>U, U>0>u$, and $0>U, u$. We thus obtain four distinct sets of conditions for vacuum stability which we summarize as follows:

$$
\begin{aligned}
& \text { (a) } \alpha_{u}, \alpha_{U} \geq 0, \quad \alpha_{U}+\alpha_{V} \geq 0, \quad \alpha_{u}+\alpha_{v} \geq 0, \\
& F\left(\alpha_{U}+\alpha_{V}\right)+\frac{\alpha_{u}+\alpha_{v}}{F}+\alpha_{w} \geq 0, \\
& \text { (b) } \alpha_{u}>0>\alpha_{U}, \quad \alpha_{U}+\frac{\alpha_{V}}{N_{\mathrm{F}}} \geq 0, \quad \alpha_{u}+\alpha_{v} \geq 0, \\
& \alpha_{U}+\frac{\alpha_{V}}{N_{\mathrm{F}}}+\frac{\alpha_{u}+\alpha_{v}}{F N_{\mathrm{f}}}+\frac{\alpha_{w}}{N_{\mathrm{f}}} \geq 0, \\
& \text { (c) } \alpha_{U}>0>\alpha_{u}, \quad \alpha_{U}+\alpha_{V} \geq 0, \quad \alpha_{u}+\frac{\alpha_{v}}{N_{\mathrm{f}}} \geq 0, \\
& \alpha_{u}+\frac{\alpha_{v}}{N_{\mathrm{f}}}+F \frac{\alpha_{U}+\alpha_{V}}{N_{\mathrm{F}}}+\frac{\alpha_{w}}{N_{\mathrm{F}}} \geq 0, \\
& \text { (d) } 0 \geq \alpha_{u}, \alpha_{U}, \quad \alpha_{U}+\frac{\alpha_{V}}{N_{\mathrm{F}}} \geq 0, \\
& \alpha_{u}+\frac{\alpha_{v}}{N_{\mathrm{f}}}+F\left(\alpha_{U}+\frac{\alpha_{V}}{N_{\mathrm{F}}}\right)+\frac{\alpha_{w}}{N_{\mathrm{F}}} \geq 0 .
\end{aligned}
$$


Notice that we have rescaled the couplings as in (26) and (27) to make contact with the notation used in this paper. The parameter $F \equiv N_{\mathrm{f}} / N_{\mathrm{F}}>0$ can be expressed in terms of the parameter $R$ to leading order in $\epsilon \ll 1$, see (45).

We make the following observations. In all four cases, the additional condition owing to the mixing coupling (27) takes the form of a lower bound for $\alpha_{w}$. Furthermore, $\alpha_{w}$ is allowed to be negative without destroying the stability of the potential, provided it does not become too negative. We also note that none of the three cases (b), (c), or (d) in (90) can have consistent solutions in the Veneziano limit where $N_{\mathrm{F}}, N_{\mathrm{f}} \rightarrow \infty$. This uniquely leaves the case (a) as the only possibility for vacuum stability in the parameter regions considered here. These solutions neatly fall back onto the solutions discussed previously in the limit $\alpha_{w} \rightarrow 0$. As long as the auxiliary condition

$$
\alpha_{w} \geq-\left[F\left(\alpha_{U}+\alpha_{V}\right)+F^{-1}\left(\alpha_{u}+\alpha_{v}\right)\right]
$$

is satisfied, we can safely conclude that a nonvanishing $\alpha_{w} \neq 0$ does not spoil vacuum stability, not even for negative portal coupling $\alpha_{w}$.

\section{Unique scalar fixed points}

In Table IX, we summarize our results for the quartic scalar couplings at all weakly interacting fixed points to leading order in $\epsilon$ following Table III, using (61). We also introduce the auxiliary functions

$$
\begin{aligned}
& F_{1}(x)=\frac{1}{4}(\sqrt{23-4 x}-1), \\
& F_{2}(x)=\frac{1}{4}(\sqrt{20-4 x+6 \sqrt{23-4 x}}-2 \sqrt{23-4 x})
\end{aligned}
$$

which originate from the scalar nullclines. The main result is that vacuum stability together with a physical fixed point in the gauge-Yukawa sector singles out a unique fixed point in the scalar sector. The scalar fixed points do not offer further parameter constraints other than those already stated in Tables VII and VIII. Within the admissible parameter ranges we invariably find that the scalar couplings are either strictly irrelevant (at interacting fixed points) or marginally irrelevant (at the Gaussian fixed point).

\section{ULTRAVIOLET COMPLETIONS}

In this section, we discuss interacting fixed points and the weak coupling phase structure of minimal models (25) in dependence on matter field multiplicities. Differences from the viewpoint of their high- and low-energy behavior are highlighted.

\section{A. Classification}

In Figs. 6-9 we summarize results for the qualitatively different types of quantum field theories with Lagrangian

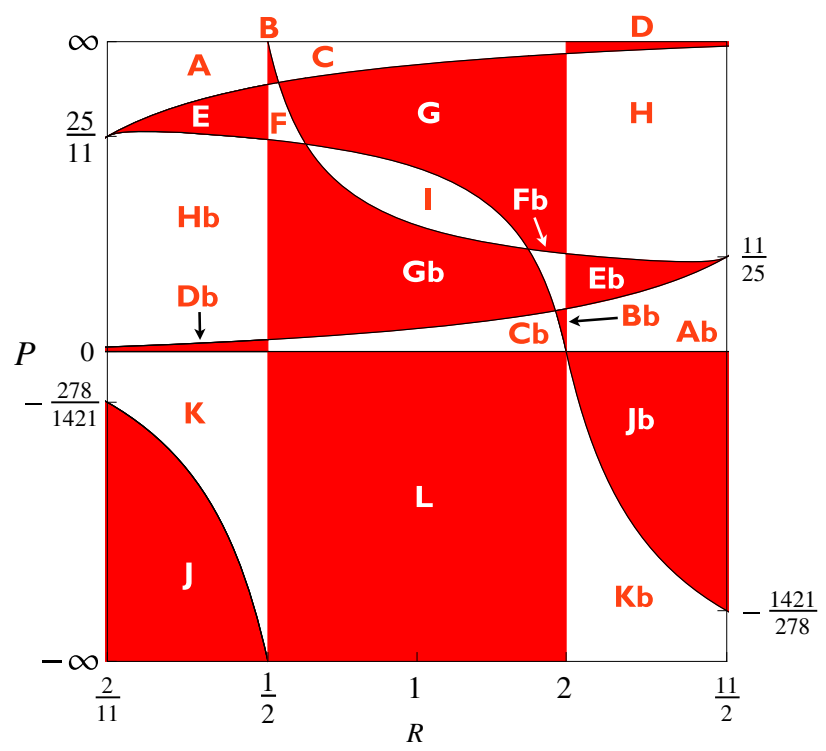

FIG. 6. The "phase space" of quantum field theories with fundamental action (25) expressed as a function of field multiplicities and written in terms of $(P, R)$, see (61). The 22 different parameter regions are indicated by roman letters. Theories with parameters in region $\mathrm{X}$ are dual to those in region $\mathrm{Xb}$ under the exchange of gauge groups following the map (46). Further details on fixed points and their eigenvalue spectra per parameter region are summarized in Figs. 7, 8, and 9.

(25) in view of their fixed point structure at weak coupling, together with their behaviour in the deep UV and IR. Theories differ primarily through their matter multiplicities (32), which translate to the parameters $(P, R)$ and the sign of $\epsilon,(61)$. As such, the "phase space" shown in Fig. 6 arises as the overlay of Figs. 1-5. Distinctive parameter regions are separated from each other by the seven characteristic curves $P=0, X, \tilde{X}, Y$ or $\tilde{Y}$ and $R=\frac{1}{2}$ or 2 . The functions $X(R), \tilde{X}(R), Y(R)$, and $\tilde{Y}(R)$ are given explicitly in (B1). Overall, this leads to the 22 distinct regions shown in Fig. 6 and denoted by capital letters. Together with the sign of $\epsilon$ this leaves us with 44 different cases. Some of these are redundant and related under the exchange of gauge groups, see (46). In fact, for $P>0$ and for either sign of $\epsilon$, we find nine fundamentally independent cases corresponding to the parameter regions

$$
\text { A, B, C, D, E, F, G, H, I }
$$

given in Fig. 6. Theories with parameters in the regime

$$
\mathrm{Ab}, \mathrm{Bb}, \mathrm{Cb}, \mathrm{Db}, \mathrm{Eb}, \mathrm{Fb}, \mathrm{Gb}, \mathrm{Hb},
$$

are "dual" to those in (93) under the exchange of gauge groups $(\mathrm{X} \leftrightarrow \mathrm{Xb})$ and for the same sign of $\epsilon$, except for the theories within (I, $\epsilon$ ), which are "self-dual" and mapped onto themselves under (46). For $P<0$ we find five parameter regions for either sign of $\epsilon$, 


$$
\mathrm{J}, \mathrm{K}, \mathrm{L}, \mathrm{Kb}, \mathrm{Jb} \text {. }
$$

For these, the manifest "duality" under exchange of gauge groups involves a change of sign for $\epsilon$ with (X, $\epsilon<0)$ being dual to $(\mathrm{Xb},-\epsilon>0)$ except for the parameter region $\mathrm{L}$ which is self-dual. In total, we end up with $2 \times 9+5=23$ fundamentally distinct scenarios underneath the $2 \times(9+8+5)=44$ cases tabulated in Figs. 7, 8, and 9 and discussed more extensively below.

A comment on the nomenclature: in each row of Figs. 7, 8 , and 9 , we indicate the parameter region $(P, R)$ as in Fig. 6 together with the sign of $\epsilon$ (if required), followed by the set of fixed points. For each of these, the (marginally) relevant and irrelevant eigenvalues in the gauge-Yukawa sector are indicated by a - and + sign. For the Gaussian fixed point $\mathrm{FP}_{1}$, the signs relate pairwise to the $S U\left(N_{\mathrm{C}}\right)$ and $S U\left(N_{\mathrm{c}}\right)$ gauge sector, respectively; for all other fixed points eigenvalues are sorted by magnitude. Red shaded slots indicate eigenvalue spectra which uniquely arise due to the semisimple character of the theory. The column "UV" states the UV fixed point, differentiating between complete asymptotic freedom (AF), asymptotic safety (AS), asymptotic freedom in one sector without asymptotic safety in the other (pAF), asymptotic safety in one sector without asymptotic freedom in the other (pAS), or none of the above. The column "IR" states the fully attractive IR fixed point (provided it exists), distinguishing the cases where none $(0)$, one $(Y)$ or $(y)$, or both (Yy) Yukawa couplings are nontrivial at the fixed point; a hyphen indicates that the IR regime is strongly coupled.

\section{B. Asymptotic freedom}

We discuss main features of the different quantum field theories (25) starting with those where each gauge sector is asymptotically free from the outset $(P>0>\epsilon)$, corresponding to the cases 1-17 in Fig. 7. The Gaussian fixed point $\mathrm{FP}_{1}$ is always the UV fixed point. Any other weakly interacting fixed point displays a lower number of relevant directions. All weakly interacting fixed points can be reached from the Gaussian. Another point in common is that all theories are completely asymptotically free meaning that-besides the gauge and the Yukawa couplings-all quartics reach the Gaussian UV fixed point.

Differences arise as to the set of interacting fixed points, summarized in Fig. 7. Overall, theories display between three and eight distinct weakly interacting fixed points. The partial Banks-Zaks fixed points $\left(\mathrm{FP}_{2}, \mathrm{FP}_{3}\right)$ are invariably present in all 17 cases. This is a consequence of a general theorem established in [34], which states that the two loop gauge coefficient is strictly positive for any gauge theory in the limit where the one-loop coefficient vanishes. This guarantees the existence of a partial Banks-Zaks fixed point in either gauge sector. At least one of the partial gaugeYukawa fixed points $\left(\mathrm{FP}_{4}, \mathrm{FP}_{5}\right)$ also arises in all cases. Moreover, the fully interacting Banks-Zaks $\left(\mathrm{FP}_{6}\right)$ as well as the fully interacting gauge-Yukawa fixed points $\left(\mathrm{FP}_{7}, \mathrm{FP}_{8}\right.$, $\mathrm{FP}_{9}$ ) are present in many, though not all, cases. All nine

\begin{tabular}{|c|c|c|c|c|c|c|c|c|c|c|c|c|c|c|}
\hline ชี & $\begin{array}{l}\text { : } \\
\text { dु } \\
\text { d్ }\end{array}$ & & & comp & $\begin{array}{l}\text { Lete as } \\
\text { (eps }\end{array}$ & $\begin{array}{l}\text { symptot } \\
<0, \text { P }\end{array}$ & $\begin{array}{l}\text { tic fr } \\
(>0)\end{array}$ & om & & & UV & & IF & \\
\hline & & G.G & $B Z . G$ & G.BZ & GY.G & G.GY & $\mathrm{BZ} \cdot \mathrm{BZ}$ & GY.BZ & BZ.GY & 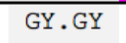 & & & $\begin{array}{l}Y \\
\end{array}$ & $\mathrm{Y} Y \mathrm{Y}$ \\
\hline 1 & A & $1---$ & $2---+$ & $3-+++$ & & $5++++$ & & $7--++$ & & $9-+++$ & 1 & 3 & 35 & 55 \\
\hline 2 & B & $1----$ & $2---+$ & $3-+++$ & $4++++$ & $5++++$ & & $7--++$ & & $9-+++$ & 1 & 3 & 45 & 54,5 \\
\hline 3 & C & $1---$ & $2---+$ & $3-+++$ & $4--+++$ & $5++++$ & & & & & 1 & 3 & 35 & 55 \\
\hline 4 & D & $1---$ & $2---+$ & $3-+++$ & $4--++$ & & & & & & 1 & 3 & 3. & $-\quad-$ \\
\hline 5 & $\mathbf{E}$ & $1---$ & $2---+$ & $3---+$ & & $5++++$ & $6--++$ & & & $9-+++$ & 1 & 6 & -5 & 55 \\
\hline 6 & $\mathbf{F}$ & $1---$ & $2---+$ & $3---+$ & $4++++$ & $5++++$ & $6--++$ & & & $9-+++$ & 1 & 6 & 45 & 54, \\
\hline 7 & G & $1---$ & $2---+$ & $3---+$ & $4--++$ & $5++++$ & $6--++$ & $7-+++$ & & & 1 & 6 & 75 & 55 \\
\hline 8 & H & $1----$ & $2---+$ & $3---+$ & $4--++$ & & $6--++$ & $7-+++$ & & & 1 & 6 & 7 & $-\quad-$ \\
\hline 9 & I & $1---$ & $2---+$ & $3---+$ & $4--++$ & $5--++$ & $6--++$ & $7-+++$ & $8-+++$ & $9++++$ & 1 & 6 & 78 & 89 \\
\hline 10 & $\mathrm{Hb}$ & $1----$ & $2---+$ & $3---+$ & & $5--++$ & $6--++$ & & $8-+++$ & & 1 & 6 & $-\varepsilon$ & $8-$ \\
\hline 11 & $\mathrm{~Gb}$ & $1---$ & $2---+$ & $3---+$ & $4++++$ & $5--++$ & $6--++$ & & $8-+++$ & & 1 & 6 & 48 & 84 \\
\hline 12 & $\mathrm{Fb}$ & $1---$ & $2---+$ & $3---+$ & $4++++$ & $5++++$ & $6--++$ & & & $9-+++$ & 1 & 6 & 45 & 54,5 \\
\hline 13 & $\mathrm{~Eb}$ & $1---$ & $2---+$ & $3---+$ & $4++++$ & & $6--++$ & & & $9-+++$ & 1 & 6 & $4-$ & -4 \\
\hline 1. & $\mathrm{Db}$ & $1---$ & $2-+++$ & $3---+$ & & $5--++$ & & & & & 1 & 2 & -2 & $2-$ \\
\hline 15 & $\mathrm{Cb}$ & $1---$ & $2-+++$ & $3---+$ & $4++++$ & $5--++$ & & & & & 1 & 2 & 4 & 44 \\
\hline 16 & $\mathrm{Bb}$ & $1---$ & $2-+++$ & $3---+$ & $4++++$ & $5++++$ & & & $8--++$ & $9-+++$ & 1 & 2 & 45 & 54, \\
\hline 17 & $\mathrm{Ab}$ & $1----$ & $2-+++$ & $3---+$ & $4++++$ & & & & $8--++$ & $9-+++$ & 1 & 2 & 24 & 44 \\
\hline
\end{tabular}

FIG. 7. Shown are the fixed points and eigenvalue spectra of quantum field theories with Lagrangean (25) for the 17 parameter regions with $\epsilon<0$ and $P>0$ in Fig. 6. Scalar selfinteractions are irrelevant at fixed points. All cases display complete asymptotic freedom in the UV. Red shaded slots indicate eigenvalue spectra which arise due to the semisimple character of the theory. In the deep IR, various types of interacting conformal fixed points are achieved depending on whether both, one, or none of the Yukawa couplings $Y$ and $y$ vanish (from left to right). Regimes with "strong coupling only" in the IR are indicated by a hyphen. 
distinct fixed points are available in the "most symmetric" parameter region I (case 9).

It is noteworthy that many theories display a fully IR attractive "sink", invariably given by an IR gauge-Yukawa fixed point in one $\left(\mathrm{FP}_{4}, \mathrm{FP}_{5}\right)$ or both gauge sectors $\left(\mathrm{FP}_{9}\right)$. In Fig. 6, this happens for matter field multiplicities in the regions A, B, C, E, F, G, I and their duals (cases 1, 2, 3, 5, 6, 7, 9, 11, 12, 13, 15, 16 and 17 of Fig. 7).

At $\mathrm{FP}_{9}$, the fully IR attractive fixed point is largely a consequence of IR attractive fixed points in each gauge sector individually. This is not altered qualitatively by the semisimple nature of the model. As such, a fully IR attractive fixed point $\mathrm{FP}_{9}$ also arises in the "direct product" limit where the $\psi$ fermions are removed.

At $\mathrm{FP}_{4}$ and $\mathrm{FP}_{5}$, in contrast, the IR sink is a direct consequence of the semisimple nature of the theory in that it would be strictly absent as soon as the messenger fermions $\psi$ are removed. Most importantly, the IR gauge Yukawa fixed point in one gauge sector changes the sign of the effective one loop coefficient in the other, mediated via the $\psi$ fermions. This secondary effect means that one gauge sector becomes IR free dynamically, rather than remaining UV free. Overall, the fixed point becomes IR attractive in all canonically marginal couplings (including the quartic couplings). In most cases the IR sink is unique except in parameter regions $\mathrm{B}$ and $\mathrm{F}$ (case 2, 6, 12, and 16) where we find two competing and inequivalent IR sinks $\left(\mathrm{FP}_{4}\right.$ versus $\left.\mathrm{FP}_{5}\right)$.

Provided that one or both Yukawa couplings take Gaussian values, other fixed points may take over the role of IR "sinks." In these settings, one or both of the elementary "meson" fields remain free for all scales and decouple from the outset. Specifically, the IR sink is given by $\mathrm{FP}_{6}$ provided that $y=0=Y$ (cases 5-13); by $\mathrm{FP}_{2}$ or $\mathrm{FP}_{7}$ provided that $y=0$ (cases 14 or $7-9$, respectively); and by $\mathrm{FP}_{3}$ or $\mathrm{FP}_{8}$ provided that $Y=0$ (cases 4 or 9-11).
We note that $\mathrm{FP}_{6}, \mathrm{FP}_{7}$ and $\mathrm{FP}_{8}$ are natural IR sinks, with or without $\psi$ fermions, provided that all Yukawa couplings of those fermions which interact with the Banks-Zaks fixed point(s) vanish. On the other hand, the result that $\mathrm{FP}_{2}$ and $\mathrm{FP}_{3}$ may become IR sinks is a strict consequence of the $\psi$ fermions and would not arise otherwise. Once more, one of the gauge sectors becomes IR free owing to the BZ fixed point in the other, an effect which is mediated via the $\psi$ fermions. In the presence of nontrivial Yukawa couplings, no fully IR stable fixed point arises for theories with field multiplicities in the parameter regions D and $\mathrm{H}$ (case 4, 8, 10 , and 14). Generically, trajectories will then run towards strong coupling with e.g. confinement or stronglycoupled IR conformality. Analogous conclusions hold true in settings with fully attractive IR fixed points provided their basins of attraction do not include the Gaussian.

Finally, another interesting feature which is entirely due to the semisimple nature of the theory are models where $\mathrm{FP}_{9}$ has a single relevant direction (cases 1, 2, 5, 6, 12, 13, 16 , and 17). Whenever this arises, the theory also always displays a fully IR attractive fixed point $\left(\mathrm{FP}_{4}, \mathrm{FP}_{5}\right.$, or both).

\section{Asymptotic safety}

We now turn to quantum field theories with (25) where asymptotic safety is realized. Asymptotic safety relates to settings where some or all couplings take non-zero values in the UV [34]. A prerequisite for this is the absence of asymptotic freedom in at least one of the gauge sectors. We find two such examples provided $P<0$ (cases 22 and 23 in Fig. 8), corresponding precisely to settings where one gauge sector is QCD-like whereas the other is QED-like. For these theories, we furthermore find that all other interacting fixed points are also present, except those of the Banks-Zaks type involving the QED-like gauge sector.

\begin{tabular}{|c|c|c|c|c|c|c|c|c|c|c|c|c|c|c|c|c|}
\hline \multirow[t]{2}{*}{ עू } & \multirow[t]{2}{*}{ 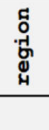 } & \multirow[t]{2}{*}{ 罗 } & \multicolumn{3}{|c|}{ asymptotic s } & \multicolumn{3}{|c|}{$\begin{array}{l}\text { ty and effective } \\
(P<0)\end{array}$} & \multicolumn{2}{|c|}{ theories } & \multicolumn{2}{|r|}{ UV } & \multicolumn{4}{|c|}{ IR } \\
\hline & & & G.G & $B Z . G$ & G.BZ & GY.G & G.GY & GY.BZ & BZ.GY & GY.GY & & & 0 & $\mathrm{Y}$ & $\mathrm{y}$ & Yy \\
\hline 18 & $J$ & - & $1--++$ & $2-+++$ & & & & & & & & (1 $\mathrm{pAF}$ ) & 2 & - & 2 & - \\
\hline 19 & $\mathbf{K}$ & - & $1--++$ & $2-+++$ & & & & & & & & (1 $\mathrm{pAF}$ ) & 2 & - & 2 & - \\
\hline 20 & L & - & $1--++$ & $2-+++$ & & $4++++$ & & & & & & (1 $\mathrm{pAF}$ ) & 2 & 4 & 2 & 4 \\
\hline 21 & $\mathrm{~K} b$ & - & $1--++$ & $2-+++$ & & $4++++$ & $5-+++$ & & & & (1 & $\mathrm{pAF}, 5 \mathrm{pAS})$ & 2 & 4 & 2 & 4 \\
\hline 22 & $\mathrm{Jb}$ & - & $1--++$ & $2-+++$ & & $4++++$ & $5---+$ & & $8--++$ & $9-+++$ & & $5 \mathrm{AS}$ & 2 & 4 & 2 & 4 \\
\hline 23 & $\mathrm{~J}$ & + & $1++--$ & & $3-+++$ & $4---+$ & $5++++$ & $7--++$ & & $9-+++$ & & $4 \mathrm{AS}$ & 3 & 3 & 5 & 5 \\
\hline 24 & $\mathbf{K}$ & + & $1++--$ & & $3-+++$ & $4-+++$ & $5++++$ & & & & (1 & $\mathrm{pAF}, 4 \mathrm{pAS})$ & 3 & 3 & 5 & 5 \\
\hline 25 & L & + & $1++--$ & & $3-+++$ & & $5++++$ & & & & & (1 $\mathrm{pAF})$ & 3 & 3 & 5 & 5 \\
\hline 26 & $\mathrm{~Kb}$ & + & $1++--$ & & $3-+++$ & & & & & & & (1 $\mathrm{pAF}$ ) & 3 & 3 & - & - \\
\hline 27 & $\mathrm{Jb}$ & + & $1++--$ & & $3-+++$ & & & & & & & (1 $\mathrm{pAF})$ & 3 & 3 & - & - \\
\hline
\end{tabular}

FIG. 8. Same as Fig. 7, covering the 10 parameter regions with $P<0$ of Fig. 6. Notice that $\mathrm{FP}_{6}$ is absent throughout. Exact asymptotic safety (AS) is realized in the cases 22 and 23. Red shaded slots indicate eigenvalue spectra which arise due to the semisimple character of the theory. For the cases 18-21 and 24-27, partial asymptotic freedom (pAF) or partial asymptotic safety (pAS) is observed whereby one gauge sector decouples entirely at all scales. The latter theories are only UV complete in one of the two gauge sectors and must be viewed as effective rather than fundamental. 
More specifically, in case 22 the role of the asymptotically safe UV fixed point is now taken by $\mathrm{FP}_{5}$. The UV critical surface is three-dimensional, in distinction to asymptotically free settings where it is four-dimensional. This reduction, ultimately a consequence of an interacting fixed point in one of the Yukawa couplings, leads to enhanced predictivity of the theory. The Gaussian necessarily becomes a cross-over fixed point with both attractive and repulsive directions, similar to the interacting $\mathrm{FP}_{8}$. Also, $\mathrm{FP}_{2}$ and $\mathrm{FP}_{9}$ are realized with a one-dimensional critical surface. The fully IR attractive $\mathrm{FP}_{4}$-the counterpart of the UV fixed point $\mathrm{FP}_{5}$ - takes the role of an IR sink. In the low energy limit, the theory displays free $S U\left(N_{c}\right)$ gluons in one gauge sector and weakly interacting $S U\left(N_{C}\right)$ gluons in the other. Moreover, the spectrum includes both free and weakly interacting mesons related to the former and the latter sectors, as well as free and weakly interacting fermions. Qualitatively, a similar result arises in the direct product limit, showing that the semisimple nature of (25) is not crucial for this scenario.

A noteworthy feature of semisimple theories with asymptotic safety is that they connect an interacting UV fixed point with an interacting IR fixed point. Hence, our models offer examples of quantum field theories with exact UV and IR conformality, strictly controlled by perturbation theory for all scales. In the massless limit, the phase diagram has trajectories connecting the interacting UV fixed point with the interacting IR fixed point. Some trajectories may escape towards the regime of strong coupling where the theory is expected to display confinement, possibly infrared conformality. The same picture arises in case 23 after exchange of gauge groups.

No asymptotically safe fixed point arises if both gauge sectors are IR free $(P, \epsilon>0)$. This result is in marked contrast to findings in the direct product limit where models with an interacting UV fixed points exist-simply because it exists for the simple gauge factors (63) and (67), given suitable matter field multiplicities. We conclude that it is precisely the semisimple nature of the specific set of theories (25) which disallows asymptotic safety for settings with $P, \epsilon>0$, see (61).

\section{Effective field theories}

We now turn to quantum field theories with (25) which are not UV complete semisimple gauge theories and, as such, must be seen as effective field theories. We find three different types of these. First, we find models with partial asymptotic freedom (pAF), where one gauge sector remains asymptotically free whereas the other stays infrared free. These models always realize a Banks-Zaks fixed point (as they must), and some also realize an IR gaugeYukawa fixed point. When viewed as a fundamental theory, the IR free sector decouples exactly, for all RG scales, and the theory becomes a simple asymptotically free gauge theory (which is UV complete). The IR-free sector

\begin{tabular}{|c|c|c|c|c|c|c|}
\hline \multirow{3}{*}{ 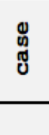 } & \multirow{2}{*}{ 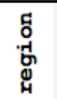 } & \multicolumn{3}{|c|}{ effective theories } & \multirow{2}{*}{ UV } & \multirow{2}{*}{$\mathbf{I R}$} \\
\hline & & (eps & $>0, \quad P$ & $>0)$ & & \\
\hline & & G.G & GY.G & G.GY & & \\
\hline 28 & $\mathbf{A}$ & $1++++$ & $4-+++$ & & (4 pAS) & 1 \\
\hline 29 & B & $1++++$ & & & none & 1 \\
\hline 30 & C & $1++++$ & & & none & 1 \\
\hline 31 & D & $1++++$ & & $5-+++$ & (5 pAS) & 1 \\
\hline 32 & $\mathbf{E}$ & $1++++$ & $4-+++$ & & (4 pAS) & 1 \\
\hline 33 & $\mathbf{F}$ & $1++++$ & & & none & 1 \\
\hline 34 & G & $1++++$ & & & none & 1 \\
\hline 35 & H & $1++++$ & & $5-+++$ & (5 pAS) & 1 \\
\hline 36 & I & $1++++$ & & & none & 1 \\
\hline 37 & $\mathrm{Hb}$ & $1++++$ & $4-+++$ & & (4 pAS) & 1 \\
\hline 38 & Gb & $1++++$ & & & none & 1 \\
\hline 39 & $\mathbf{F b}$ & $1++++$ & & & none & 1 \\
\hline 40 & $\mathrm{~Eb}$ & $1++++$ & & $5-+++$ & (5 pAS) & 1 \\
\hline 41 & $\mathrm{Db}$ & $1++++$ & $4-+++$ & & (4 pAS) & 1 \\
\hline 42 & $\mathrm{Cb}$ & $1++++$ & & & none & 1 \\
\hline 43 & $\mathrm{Bb}$ & $1++++$ & & & none & 1 \\
\hline 44 & $\mathbf{A b}$ & $1++++$ & & $5-+++$ & (5 pAS) & 1 \\
\hline
\end{tabular}

FIG. 9. Same as Figs. 7 and 8, covering the 17 parameter regions where $\epsilon>0$ and $P>0$ in Fig. 6. Asymptotic freedom is absent in both gauge sectors implying that $\mathrm{FP}_{2}, \mathrm{FP}_{3}, \mathrm{FP}_{6}, \mathrm{FP}_{7}$, and $\mathrm{FP}_{8}$ cannot arise. Partial asymptotic safety (in one gauge sector) is observed in case 28,31,32,35,37,40,41, and 44, whereby the other gauge sector remains free at all scales (pAS). All models must be viewed as effective rather than fundamental theories. All theories become trivial in the IR.

can be interacting when viewed as an effective theory, very much like the $U(1)_{Y}$ sector of the Standard Model. This setting requires $P<0$ and is realized in cases 18-21 and $23-27$.

Second, we find models with partial asymptotic safety (pAS), where one gauge sector becomes asymptotically safe whereas the other remains free at all scales. All such models display a UV gauge-Yukawa fixed point. When viewed as a fundamental theory, these semisimple gauge theories in fact reduce to a simple asymptotically safe gauge theory (which is UV complete). The IR-free sector can be interacting when viewed as a non-UV complete effective theory. This setting mostly requires $P, \epsilon>0$ and is realized in cases $28,31,32,35,37,40,41$, and 44 . Curiously, pAS is also realized in cases 21 and 24 where $P<0$ alongside pAF in the other gauge sector-such models have two disconnected UV scenarios, where we can choose to have either asymptotic freedom in one sector, or asymptotic safety in the other, in each case with the remaining sector decoupling at all scales. Once more, if both gauge sectors are interacting these models must be viewed as (non-UV complete) effective theories.

Finally, we find models with none of the above. In these settings (cases 29, 30, 33, 34, 36, 38, 39, 42 and 43), both gauge sectors are IR free and no other weakly coupled fixed points are realized, leaving us with no perturbative 
UV completion. In the cases 28-44, the Gaussian acts as in IR sink for RG trajectories. Along these, the longdistance behavior is trivial, characterized by free massless non-Abelian gauge fields, quarks, and elementary mesons.

In summary, the semisimple gauge Yukawa theories (25) have a well-defined UV limit with either asymptotic freedom or asymptotic safety in $9+1=10$ cases out of the 23 fundamentally distinct parameter settings covered in Fig. 6 . The remaining $4+9=13$ parameter settings do not offer a well-defined UV limit at weak coupling. This completes the classification of the models with (25).

\section{PHASE DIAGRAMS OF GAUGE THEORIES}

In this section, we discuss the phase diagrams of UV complete theories of the type (25), particularly in view of theories with asymptotic freedom or asymptotic safety.

\section{A. Semisimple gauge theories without Yukawas}

We begin with settings where Yukawa couplings are switched off. In these cases, interacting fixed points can only arise for asymptotically free gauge sectors, and fixed points are of the Banks-Zaks type or products thereof
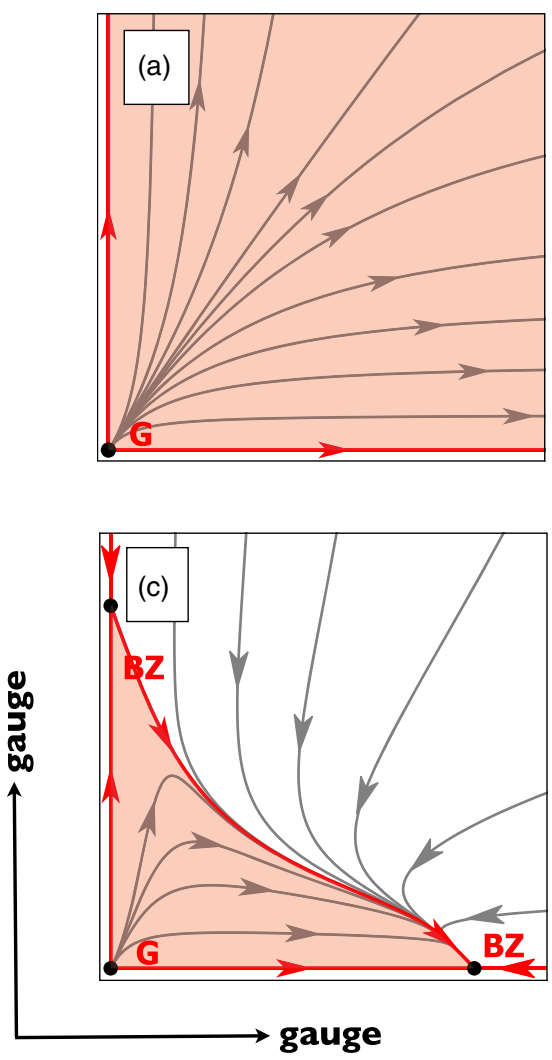

[34,35]. Qualitatively different cases realized amongst the theories (25) are summarized in Fig. 10 for semisimple gauge theories with two gauge groups $G_{1} \times G_{2}$. Results generalize to more gauge groups in an obvious manner.

Specifically, Fig. 10(a) shows theories with asymptotic freedom but without any BZ fixed points. UV free trajectories emanate out of the Gaussian fixed point and invariably escape towards strong coupling where the theory is expected to display confinement, or IR conformality. Similarily, Fig. 10(b) shows theories with asymptotic freedom and a BZ fixed point in one of the gauge sectors. The other gauge coupling remains an IR relevant perturbation even at the BZ. Therefore UV free trajectories will again escape towards strong coupling in the IR.

Figure 10(c) shows asymptotic freedom with a BZ fixed point in both gauge sectors individually. Here, and much unlike Fig. 10(b), one of the BZ fixed points has turned into an exact IR sink, and both BZ fixed points are connected by a separatrix. As we have already noticed in Sec. VI B, the presence of an interacting fixed point in one gauge sector can turn the other gauge sector from UV free to IR free. This new type of phenomenon has become possible owing to the $\psi$ fermions and is once again due to the semisimple
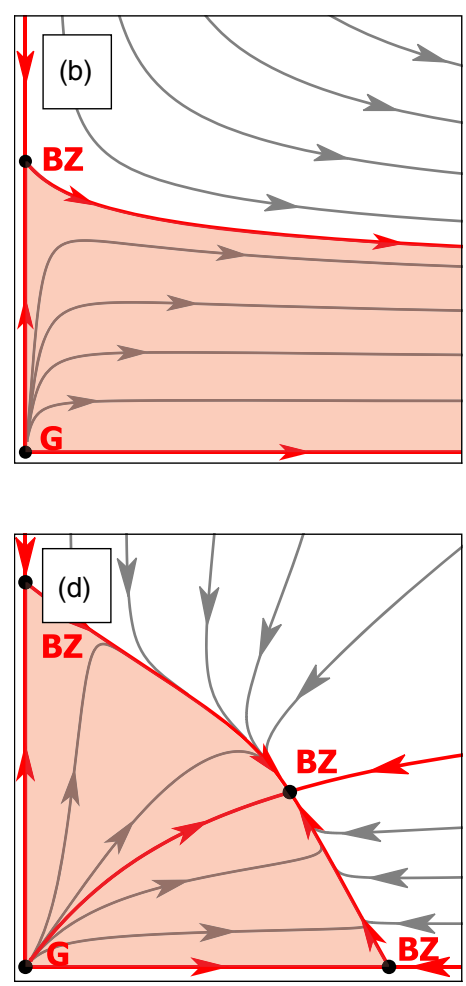

FIG. 10. Phase diagrams of asymptotically free semisimple gauge theories (two gauge groups) coupled to matter without Yukawas, covering (a) asymptotic freedom and the Gaussian $(\mathrm{G})$ without interacting fixed points and trajectories running towards strong coupling and confinement, (b) the same, with an additional Banks-Zaks (BZ) fixed point, (c) two BZ fixed points, one of which turned into an IR sink for all trajectories, or (d) three BZ fixed points, the fully interacting one now becoming the IR sink. Axes show the running gauge couplings, fixed points (black) are connected by separatrices (red), and red-shaded areas cover all UV free trajectories with arrows pointing from the UV to the IR. 
nature of the theory. Therefore, all UV free trajectories invariably are attracted into the IR sink. In the deep IR, the theory approaches a conformal fixed point with massless and unconfined free and weakly coupled gluons and quarks. Regimes of strong coupling cannot be reached.

Figure 10(d) shows asymptotic freedom with a (partial) BZ fixed point in either gauge sector individually, as well as a fully interacting BZ fixed point. Most notably, all UV free trajectories are attracted by the later, which acts as an IR sink. No trajectories can escape towards strong coupling. The long distance physics is characterized by an interacting conformal field theory with massless weakly coupled gauge fields and fermions. Here, and unlike in Fig. 10(c), all fields remain weakly coupled in the IR.

In the scenarios of Figs. 10(a) and 10(b) UV free trajectoires run towards strong coupling and confinement in the IR, in one or both gauge sectors. In contrast, the scenarios in Figs. 10(c) and 10(d) show that all UV free trajectories are attracted by an IR-stable conformal fixed point. These theories remain unconfined and perturbative at all scales. All four scenarios in Fig. 10 are realized for our template of semisimple gauge theories with Lagrangean
(25). Explicit examples are given for models without Yukawa couplings $(Y=0=y)$ and for field multiplicities in the parameter regions (a) $\epsilon_{1}, \epsilon_{2}<-75 / 26$, (b) $\epsilon_{1}<$ $-75 / 26$ and $-75 / 26<\epsilon_{2}<0$, or $\left(\epsilon_{1} \leftrightarrow \epsilon_{2}\right)$, (c) the cases 1-4 and 14-17 of Fig. 7, and (d) the cases 5-13 of Fig. 7.

\section{B. Simple gauge theories with Yukawas}

We continue the discussion of phase diagrams with simple gauge theories with gauge group $G$ and a single Yukawa coupling. Four distinct cases can arise [34,35], summarized in Fig. 11. For asymptotically free settings, the theory either shows (a) only the Gaussian UV fixed point, (b) the Gaussian together with the Banks-Zaks, or (c) the Gaussian together with the Banks-Zaks and an IR gaugeYukawa fixed point. Simple gauge theories can also become asymptotically safe, in which case (d) a UV gauge-Yukawa fixed point arises. Trajectories are directed towards the IR. The red-shaded areas indicate the set of UV complete trajectories emanating out of the UV fixed point. We genuinely observe a two-dimensional area of trajectories for asymptotically free settings, which is reduced to a onedimensional set in the asymptotically safe scenario. The IR
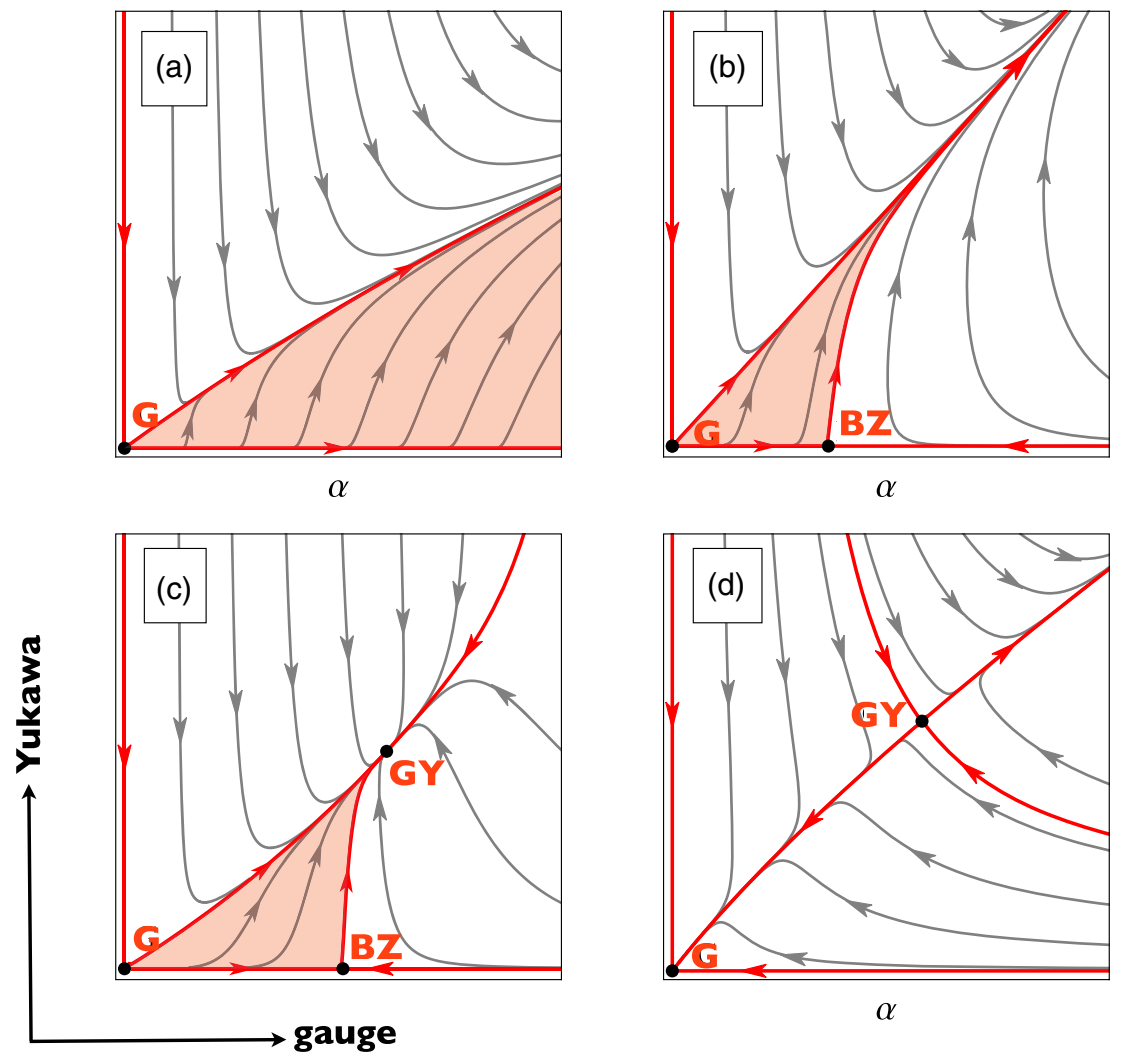

FIG. 11. Phase diagrams of UV complete and weakly interacting simple gauge theories coupled to matter with a single Yukawa coupling, covering (a) asymptotic freedom with the Gaussian UV fixed point and no other weakly interacting fixed point, (b) asymptotic freedom with a Banks-Zaks (BZ) fixed point, (c) asymptotic freedom with a Banks-Zaks and an IR gauge-Yukawa (GY) fixed point, and (d) asymptotic safety with an UV gauge-Yukawa fixed point. Axes display the running gauge and Yukawa couplings, fixed points (black) are connected by separatrices (red), and red-shaded areas cover all UV free trajectories with arrows pointing from the UV to the IR [34,35]. Examples are given by (63), (67) (see main text). 


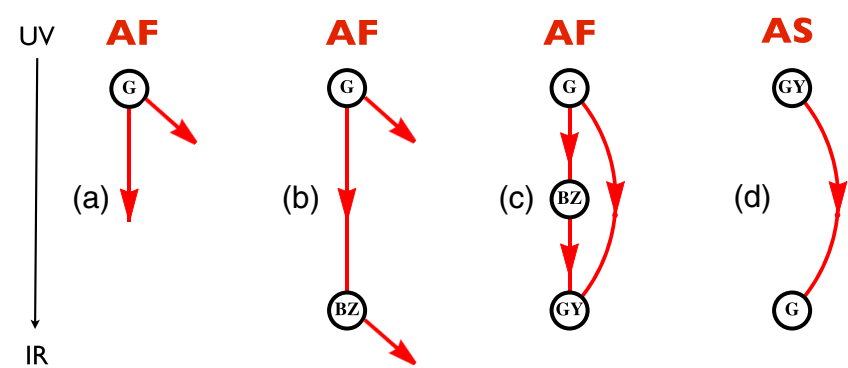

FIG. 12. "Primitives" for phase diagrams of simple gaugeYukawa theories with asymptotic freedom (AF) or asymptotic safety (AS), corresponding to the different setting shown in Fig. 11. Arrows point from the UV to the IR and connect the different fixed points. Open arrows point towards strong coupling in the IR. The number of outgoing red arrows gives the dimensionality of the UV critical surface. The separate UV safe trajectory towards strong coupling in case (d) is not indicated. Yukawa-induced IR unstable directions in $(a, b)$ or gauge Yukawa fixed points in (c,d) are absent as soon as Yukawa interactions are switched off from the outset.

regime is characterized by either strong interactions and confinement such as in Figs. 11(a,b,d), or by an interacting conformal field theory with weakly coupled gluons and fermions alongside free or interacting scalar mesonscorresponding to the BZ fixed points in Figs. 11(b) and 11(c), or the IR GY fixed point in Fig. 11(c), respectivelyor by Gaussian scaling, Fig. 11(d).

All four scenarios in Fig. 11 are realized for simple gauge theories with (63) corresponding to the parameter regions (a) $\epsilon_{1}<-75 / 26$, (b) $-75 / 26<\epsilon_{1}<0$ and $R>\frac{1}{2}$, (c) $-75 / 26<\epsilon_{1}<0$ and $R<\frac{1}{2}$, or (d) $\epsilon_{1}>0$ and $R<\frac{1}{2}$, respectively, with $R$ additionally bounded by (64).

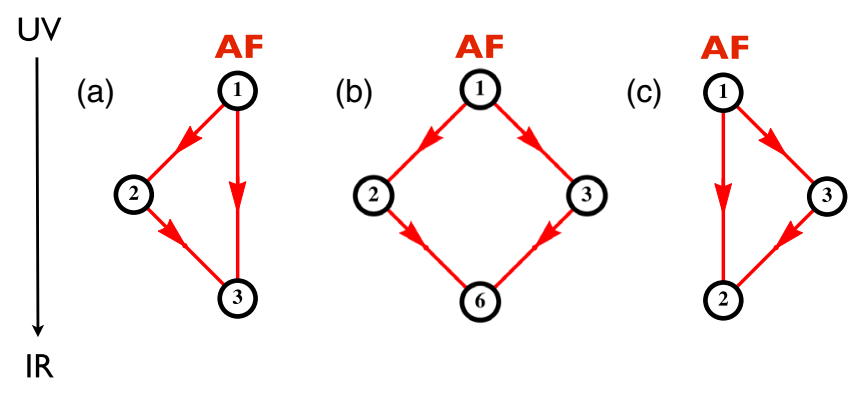

FIG. 13. Schematic phase diagram for asymptotically free semisimple gauge theories (25) with Banks-Zaks type fixed points without Yukawas and exact IR conformality. Field multiplicities correspond to the cases (a) 1-4, (b) 5-13, and (c) 14-17 of Fig. 7, respectively, with scalars decoupled. RG flows point from the UV to the IR (top to bottom). At each fixed point, the dimensionality of the UV critical surface is given by the number of outgoing red arrows. All UV free trajectories terminate at $\mathrm{FP}_{2}, \mathrm{FP}_{6}$, and $\mathrm{FP}_{3}$, respectively, which act as fully attractive IR sinks. The topology of the phase diagram (b) is the "square" of Fig. 12(b), representing Fig. 10(d). The phase diagrams (a) and (c), representing Fig. 10(c), cannot be constructed from the primitives in Fig. 12.
An economic way to display phase diagrams for semisimple theories with or without Yukawas is achieved by introducing a schematic diagrammatic language, see Fig. 12. Each of the four basic phase diagrams in Fig. 11 are represented by a "primitive" diagram, Fig. 12, where full dots indicate (free or interacting) fixed points, red arrows indicate the outgoing trajectories, and RG flows schematically run "top-down" from the UV to the IR. Also, at each fixed point the number of outgoing arrows indicates the dimensionality of the fixed point's "UV critical surface." Fixed points are connected by separatrices. We use straight lines to indicate separatrices involving the BZ fixed point, curved lines to indicate separatrices connecting GY fixed points with the Gaussian, and open-ended lines to denote RG trajectories running towards strong coupling without reaching any weakly coupled fixed points.

Specifically, in case (a), a two-dimensional array of RG flows are running out of the Gaussian UV fixed point towards strong coupling, with no weakly interacting fixed points. In case (b), we additionally observe a Banks-Zaks fixed point. It is connected with the Gaussian by a separatrix shown in red. Arrows invariably point towards the IR. Yukawa couplings act as an unstable direction at both fixed points. In case (c), we additionally observe a gauge-Yukawa fixed point besides the Gaussian and the BZ. All three fixed points are connected by separatrices. Note that two lines emanate from the Gaussian, reflecting that the UV critical surface is two dimensional. The GY fixed point arises as an IR sink, which attracts all UV-free trajectories emanating out of the Gaussian. In case (d), the model is asymptotically safe and the GY fixed point has become the interacting UV fixed point. A Banks-Zaks fixed point can no longer arise [34]. The theory has a one-dimensional UV critical surface connecting the GY fixed point with the IR Gaussian fixed point via a separatrix. A second UV safe trajectory which leaves the GY fixed point towards strong coupling is not depicted. Finally, we note that the Yukawa-induced IR unstable directions in (a) and (b) or gauge Yukawa fixed points in (c) and (d) are absent as soon as Yukawa interactions are switched off from the outset.

\section{Semisimple gauge theories with asymptotic freedom}

We consider phase diagrams for semisimple theories (25) with complete asymptotic freedom, exemplified by all models in Fig. 7. When Yukawa couplings are absent, the mesonlike scalar degrees of freedom remain free at all scales and decouple from the theory. In the regime with asymptotic freedom solely Banks-Zaks fixed points can arise in the IR. Figures 10(d) and 13(b) shows settings where all Banks-Zaks fixed points are present, corresponding to the cases $5-13$ of Fig. 7. RG flows point from the UV to the IR (top to bottom) and connect the Gaussian UV fixed point $\left(\mathrm{FP}_{1}\right)$ with either of the partially $\left(\mathrm{FP}_{2}\right.$ and $\left.\mathrm{FP}_{3}\right)$ and the fully interacting $\left(\mathrm{FP}_{6}\right)$ Banks-Zaks fixed points. The latter is fully attractive and acts as an IR sink. 


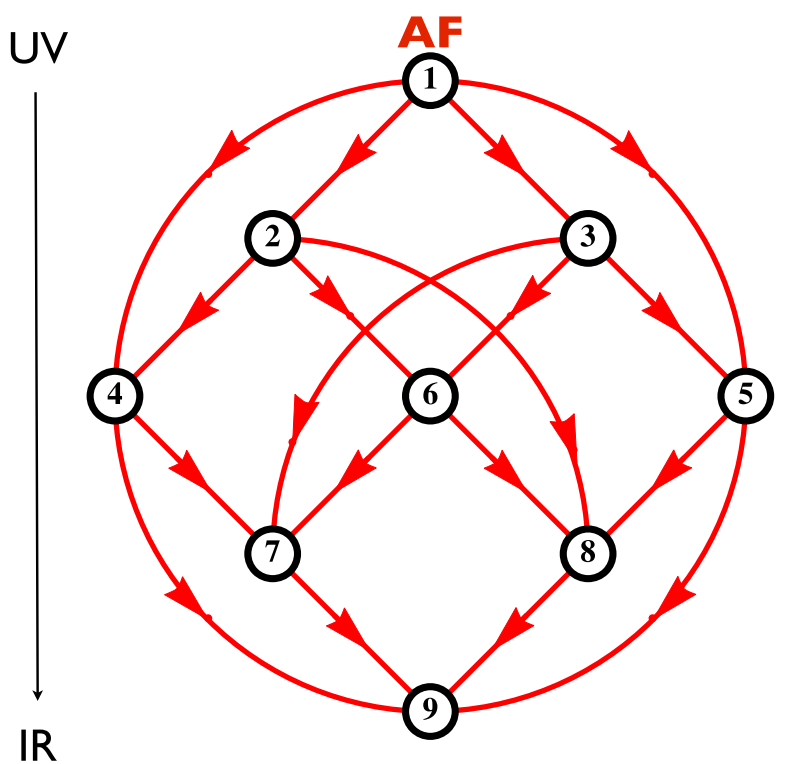

FIG. 14. Asymptotic freedom and schematic phase diagram for semisimple gauge-Yukawa theories with field multiplicities as in case 9 of Fig. 7. RG flows point from the UV to the IR (top to bottom). Besides the Gaussian UV fixed point $\left(\mathrm{FP}_{1}\right)$, the theory displays all eight weakly interacting fixed points, see Table III. At each fixed point, the dimensionality of the UV critical surface is given by the number of outgoing red arrows. $\mathrm{FP}_{9}$ is fully attractive and acts as an IR sink. The topology of the phase diagram is the square of Figs. 11, 12(c); see main text.

The topology of the phase diagram is the "square" of Figs. 11, 12(b). In the deep IR the theory is unconfined yet weakly interacting, and the elementary gauge fields $A, a$ and fermions $Q, q$ and $\psi$ appear as massless particles at the IR conformal fixed point. The phase diagrams in Figs. 13(a) and 13(c) cannot be constructed out of the simple primitives, Fig. 12. The reason for this is that the eigenvalue spectrum at one of the fixed points deviates from the direct product spectrum due to interactions.

Next we include Yukawa interactions. We have already concluded from Fig. 7 that the eigenvalue spectrum in the cases 8,9 , and 10 agrees qualitatively, for all fixed points, with the eigenvalue spectrum in the corresponding direct product limit. In these settings, we may then use the primitives in Fig. 12 to find the semisimple phase diagrams. We consider the case where the parameters (61) take values within the range I of Fig. 6 and for $\epsilon<0$, corresponding to case 9 of Fig. 7. This family of theories includes the "symmetric" setup $(R, P)=(1,1)$ where symmetry under the exchange of gauge groups is manifest. The UV fixed point is given by the Gaussian $\left(\mathrm{FP}_{1}\right)$, and the UV critical surface at the Gaussian is four-dimensional, owing to the marginal UV relevancy of the two gauge and the two Yukawa couplings. All scalar couplings are irrelevant in the UV and can be expressed in terms of the gauge and the Yukawa couplings along UV-free trajectories. Moreover, each gauge sector displays the Banks-Zaks and a gauge-

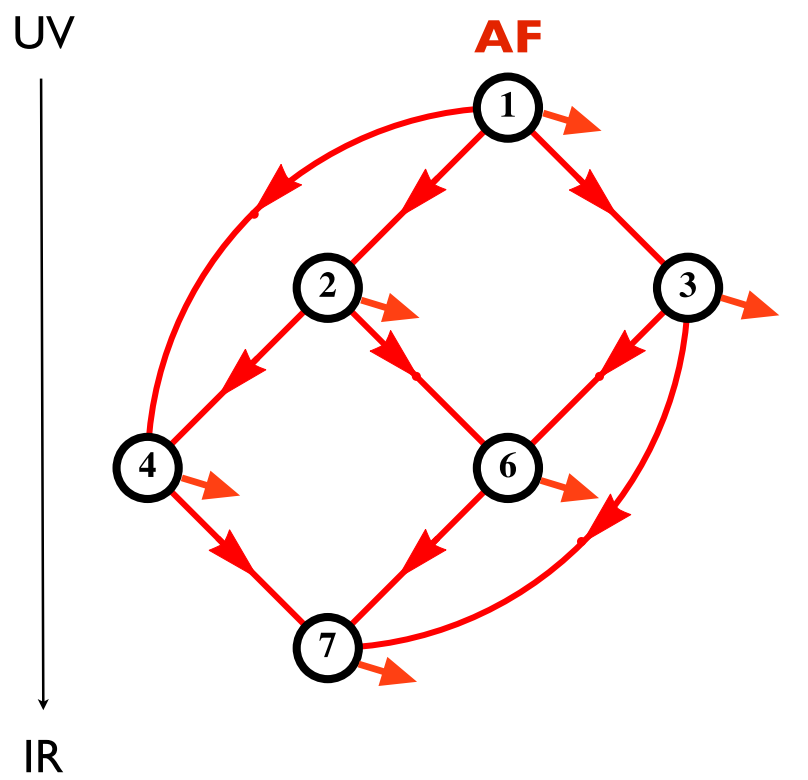

FIG. 15. Asymptotic freedom and schematic phase diagrams for semisimple gauge-Yukawa theories with field multiplicities as in case 8 of Fig. 7. Flows point from the UV to the IR (top to bottom). The theories display five weakly interacting fixed points besides the Gaussian UV fixed point $\left(\mathrm{FP}_{1}\right)$. The unavailability of $\mathrm{FP}_{5}, \mathrm{FP}_{8}$, and $\mathrm{FP}_{9}$ implies that some trajectories escape towards strong coupling (short arrows), and none of the fixed points acts as a complete IR attractor. The topology of the phase diagram is the "direct product" of Figs. 11, 12(c) with Figs. 11, 12(b); see main text. The IR unstable direction is removed provided that the Yukawa coupling $y \equiv 0$, in which case the singlet mesons $h$ decouple.

Yukawa fixed point individually, and all nine fixed points are realized in the full theory.

Since the sign pattern of the eigenvalue spectra at all fixed points is equivalent to the direct product limit, the topology of the semisimple phase diagram is the square of Fig. 12(c)—shown in Fig. 14. Fixed points are connected by separatrices (red lines), and arrows always point towards the IR. From top to bottom, the fixed points $\mathrm{FP}_{1}\left(\mathrm{FP}_{2,3}\right)$ [FP $\left.{ }_{4,5,6}\right]\left(\mathrm{FP}_{7,8}\right)$ and $\mathrm{FP}_{9}$ have a 4 (3) [2] (1) and 0-dimensional UV critical surface, respectively, corresponding to the number of outgoing red arrows. $\mathrm{FP}_{9}$ acts as an IR attractor for all trajectories within its basin of attraction. Consequently, the elementary quarks and gluons are not confined and the theory corresponds to a conformal field theory of weakly interacting massless gluons, fermions, and mesons in the deep IR. For certain fine-tuned settings, the IR limit would, instead, correspond to one of the other interacting fixed points $\mathrm{FP}_{2}-\mathrm{FP}_{8}$, relating to different conformal field theories. Also, while all other fixed points can be reached from the Gaussian $\mathrm{FP}_{1}$ (whose UV critical surface has the largest dimensionality), it is not true in general that a fixed point with a smaller UV critical dimension can be reached from a fixed point with a larger one. Fixed points are also not connected "horizontally." 
As a further example we consider a less symmetrical setting given by models with (61) in the parameter range $\mathrm{H}$ (or $\mathrm{Hb}$ ) of Fig. 6, and for $\epsilon<0$. In these theories, only one of the two gauge sectors can achieve a gauge-Yukawa fixed point. Consequently, six different types of fixed points are realized. The sign pattern of the eigenvalue spectrum (cases 8 or 10, Fig. 7) ensures that the topology of the semisimple phase diagram obtains as the direct product of Fig. 12(b) with Fig. 12(c), shown in Fig. 15. From top to bottom, the fixed points $\mathrm{FP}_{1}\left(\mathrm{FP}_{2,3}\right)\left[\mathrm{FP}_{4,6}\right]$, and $\mathrm{FP}_{7}$ have a 4 (3) [2] and 1-dimensional UV critical surface, respectively. Fixed points are connected by separatrices. The absence of $\mathrm{FP}_{5}$, $\mathrm{FP}_{8}$, and $\mathrm{FP}_{9}$ implies that some trajectories escape towards strong coupling, indicated by short arrows, from each of the fixed points. The unstable direction relates to the Yukawa coupling $y$ in (25). Provided it is switched off, $\mathrm{FP}_{7}$ would become the fully attractive IR sink. In this case, the elementary mesons $h$ are spectators and remain free at all scales. Also, the elementary quarks and gluons remain unconfined. In the deep IR, the theory corresponds to a conformal field theory of weakly interacting massless gluons $A$, fermions $Q, \psi$ and mesons $H$, together with free and massless gluons $a$, fermions $q$ and mesons $h$, see Table IV. For certain fine-tuned settings, the IR limit would, instead, correspond to one of the other interacting fixed points $\mathrm{FP}_{2}-\mathrm{FP}_{8}$, relating to different conformal field theories.

The phase diagrams of asymptotically free theories in the cases 1-7 and 11-17 of Fig. 7 cannot be constructed out of the simple primitives, Fig. 12. The reason for this is that their eigenvalue spectrum at some of the interacting fixed points deviates from the direct product spectrum. Once again this effect is due to the semisimple nature of the theory. A more detailed study of these cases is left for future work.

\section{Semisimple gauge theories with asymptotic safety}

We finally turn to the phase diagram of semisimple gauge theories with exact asymptotic safety. From Figs. 8 and 9 we conclude that asymptotic safety arises through a partially interacting UV fixed point where one gauge sector is interacting whereas the other gauge sector is free. This is achieved for matter field multiplicities (61) taking values within the range $\mathrm{J}$ or $\mathrm{Jb}$ of Fig. 6, corresponding to cases 22 or 23 of Fig. 8. Once more, the eigenvalue spectra at all fixed points are equivalent to the ones in the direct product limit, implying that the phase diagram arises as the direct product of the corresponding simple factors Figs. 11, 12(c) and Figs. 11, 12(d).

Figure 16 shows the schematic phase diagram for case 22 , where the asymptotically safe UV fixed point $\mathrm{FP}_{5}$ is of the $\mathrm{G} \cdot \mathrm{GY}$ type (see Tables III and V). Unlike the cases with asymptotic freedom, here, the UV hypercritical surface is three rather than four dimensional. The reason for this is that one of the Yukawa couplings is taking an

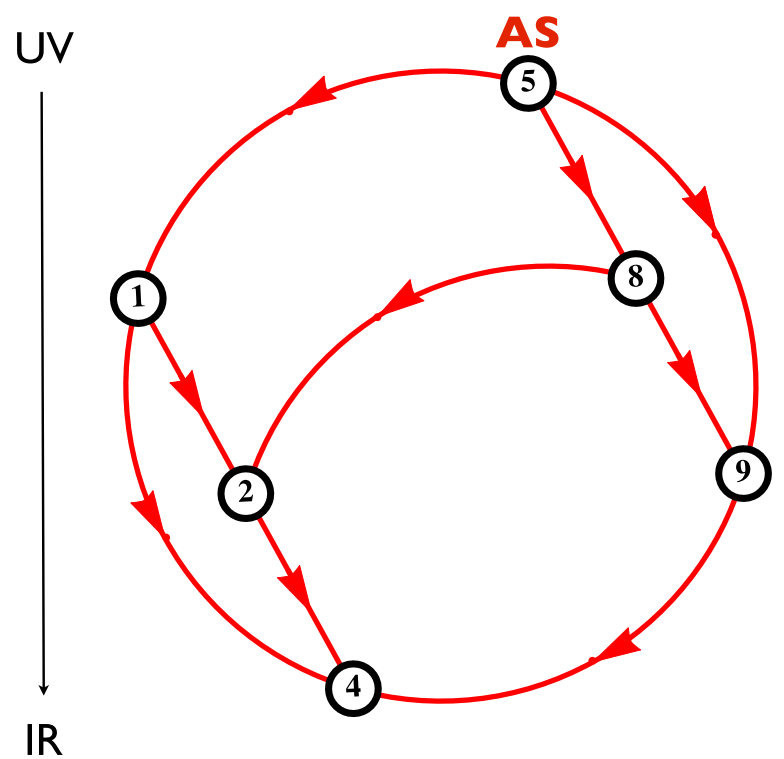

FIG. 16. Asymptotic safety and schematic phase diagram of semisimple gauge-Yukawa theories with field multiplicities as in case 22 of Fig. 8. Besides the partially interacting UV fixed point $\left(\mathrm{FP}_{5}\right)$, the theory displays five weakly interacting fixed points. The Gaussian $\left(\mathrm{FP}_{1}\right)$ takes the role of a crossover fixed point and $\mathrm{FP}_{4}$ takes the role of an IR sink. The topology of the phase diagram is the direct product of Figs. 11, 12(c) with Figs. 11, 12(d); see main text.

interacting UV fixed point. At each fixed point, the number of outgoing directions indicate the dimensionality of the fixed point's critical hypersurface. From top to bottom, the fixed points $\mathrm{FP}_{5}\left(\mathrm{FP}_{1,8}\right)\left[\mathrm{FP}_{2,9}\right]$ and $\mathrm{FP}_{4}$ have a 3 (2) [1] and 0 -dimensional UV critical surface. UV finite trajectories connect $\mathrm{FP}_{5}$ via intermediate cross-over fixed points with the fully IR attractive fixed point $\mathrm{FP}_{4}$, which is of the GY · G type. At weak coupling, all UV-IR connecting trajectories proceed either via the Gaussian $\mathrm{FP}_{1}(\mathrm{G} \cdot \mathrm{G})$ and $\mathrm{FP}_{2}(\mathrm{BZ} \cdot \mathrm{G})$, or via $\mathrm{FP}_{8}(\mathrm{BZ} \cdot \mathrm{GY})$ and $\mathrm{FP}_{2}$ or $\mathrm{FP}_{9}$ $(\mathrm{GY} \cdot \mathrm{GY})$. The Gaussian fixed point is IR free in one of the gauge couplings meaning that is necessarily arises as a cross-over fixed point. There are no trajectories connecting the fixed points $\mathrm{FP}_{1}$ with $\mathrm{FP}_{9}$ because the sole relevant direction at the latter is an irrelevant direction at the former. $\mathrm{FP}_{4}$ acts as an IR "sink" for RG trajectories. While all other fixed points can be reached from the interacting UV fixed point $\mathrm{FP}_{5}$ (whose UV critical surface has the largest dimensionality), it is not true in general that a fixed point with a smaller UV critical dimension can be reached from a fixed point with a larger one (e.g. $\mathrm{FP}_{9}$ cannot be reached from $\mathrm{FP}_{1}$ ). Fixed points are also not connected "horizontally".

An intriguing novelty of our models with asymptotic safety is that both the deep UV and the deep IR limits are characterized by weakly interacting conformal field theories. For example, in the deep UV the theories of case 22 
correspond to conformal field theories of weakly interacting massless gluons $a$, fermions $q, \psi$ and mesons $h$, together with free and massless gluons $A$, fermions $Q$ and mesons $H$. Along the UV-IR transition, the fields $(A, Q, H)$ and $(a, q, h)$ effectively "interchange" their roles, ultimately approaching conformal field theories of weakly interacting massless gluons $A$, fermions $Q, \psi$, and mesons $H$, together with free and massless gluons $a$, fermions $q$ and mesons $h$ in the IR. Hence, one may say that IR conformality in the $S U\left(N_{\mathrm{c}}\right)$ gauge sector arises from UV conformality in the $S U\left(N_{\mathrm{C}}\right)$ gauge sector through a "seesaw" mechanism transmitted via the $\psi$ fermions, i.e., the only fields which are interacting at all scales including the UV and the IR limits. For certain fine-tuned settings, the IR limit would, instead, correspond to one of the other interacting fixed points $\mathrm{FP}_{1}, \mathrm{FP}_{2}, \mathrm{FP}_{8}$, or $\mathrm{FP}_{9}$, relating to different conformal field theories. Also, for certain UV parameters, theories may escape towards strong coupling in the IR.

\section{E. Mass deformations and phase transitions}

In the vicinity of fixed points phase transitions between different phases arise once mass terms are switched on. At weak coupling mass anomalous dimensions are perturbatively small (Sec. III D). The running of scalar or fermion mass terms, once switched on, will then be dominated by their canonical mass dimensions-modulo small quantum corrections. Consequently, mass terms add additional relevant directions at all fixed points (e.g., Figs. 14-16). Each of the eight interacting UV fixed points relates to a quantum phase transition between phases with and without spontaneous breaking of symmetry where the vacuum expectation value of the scalar fields serves as an order parameter. In particular, fixed points which act as IR sinks for the canonically marginal interactions (such as $\mathrm{FP}_{9}$ in Fig. 14 and $\mathrm{FP}_{4}$ in Fig. 16) develop new unstable directions driven by the mass. Scalar fields may or may not develop vacuum expectation values leading to symmetric and symmetry broken phases, respectively. Also, fermions may acquire masses spontaneously. Thereby a variety of different phases may arise, connected by first and higher order quantum phase transitions. Close to interacting fixed points, phase transitions are continuous and, in some cases, of the Wilson-Fisher type with a single relevant parameter. We leave a more detailed investigation of phase transitions for a future study.

\section{DISCUSSION}

In this section, we address further aspects of interacting fixed points covering universality and operator ordering, triviality bounds, perturbativity in and beyond the Veneziano limit, conformal symmetry, and conformal windows.

\section{A. Gap, universality, and operator ordering}

At partially or fully interacting fixed points, the degeneracy of the nine classically marginal couplings (26), (27) is partly or fully lifted. We have computed scaling exponents to the leading nontrivial order in $\epsilon$. Interacting fixed points have nontrivial exponents of order $\sim \epsilon$, except if a gauge coupling is involved in which case one of the exponents is parametrically smaller $\sim \epsilon^{2}$. Hence, the eigenvalue spectrum opens up $\sim \epsilon$ because eigenvalues of order $\epsilon$ are invariably present at any of the interacting fixed points. It is convenient to denote the difference between the smallest negative eigenvalue and the smallest positive eigenvalue as the "gap" in the eigenvalue spectrum, which serves as an indicator for interaction strength [5,24]. Simple $S U(N)$ gauge theories in the Veneziano limit such as (63) display a gap of order $\sim \epsilon\left(\epsilon^{2}\right)$ at the Banks-Zaks or the UV gauge Yukawa (IR gauge Yukawa) fixed point, respectively [32]. In semisimple theories, and depending on the specifics of the fixed point, we again find that the gap is either of order $\epsilon$ or of order $\epsilon^{2}$. (The gap trivially vanishes if one of the gauge sectors is asymptotically free and takes Gaussian values.) The gap still depends on the remaining free parameters $(P, R)$.

Also, all results for fixed points and scaling exponents are universal and independent of the RG scheme, although we have used a specific scheme (MS bar) throughout. This is obviously correct for dimensionless couplings at one loop where divergences are logarithmic. We have checked that it also holds at two loop level both for the gauge sectors, and for the Yukawa contributions to the running of the gauge coupling(s) [32]. The field strengths and the Yukawa couplings are marginally relevant operators at asymptotically free Gaussian UV fixed points (case 1-17 of Fig. 7). At asymptotically safe UV fixed points, one of the field strengths becomes relevant and the corresponding Yukawa coupling irrelevant (case 22, 23 of Fig. 8). There is no UV fixed point where both gauge sectors remain interacting. The scalar self-interactions are (marginally) irrelevant at any fixed point.

\section{B. Elementary gauge fields and scalars}

Triviality bounds relate to perturbative UV Landau poles of infrared free interactions. They limit the predictivity of theories to a maximal UV extension [52]. For theories with action (25), perturbative UV Landau poles can arise for gauge couplings in the absence of asymptotic freedom or asymptotic safety. Examples for this are given in cases 18-21 and 24-27 of Fig. 8 where one gauge sector is IR free, as well as in cases 28-44 of Fig. 9 where both gauge sectors are IR free. In these cases the theories can at best be treated as effective rather than fundamental (see Sec. VID). Conversely, triviality in gauge sectors is trivially avoided in settings with asymptotic freedom 
(such as in cases 1-17), and nontrivially in settings with asymptotic safety (case 22 and 23). In the latter cases, the loss of asymptotic freedom is compensated through an interacting fixed point in the Yukawa and scalar couplings, which enabled a fixed point for the gauge coupling [32]. We stress that scalar fields and Yukawa interactions play a key role. Without them, triviality of any QED-like gauge theories cannot be avoided [34,35].

Triviality also relates to the difficulty of defining elementary self-interacting scalar quantum fields in four dimensions [53-55]. It is interesting to notice that the quartic scalar couplings always take a unique physical fixed points as soon as the gauge and Yukawa coupling take weakly coupled fixed points. Hence, in theories with (25) scalar fields can be viewed as elementary and triviality is evaded in all settings with asymptotic freedom and asymptotic safety. In either case gauge fields play an important role, albeit for different reasons [32]. For gauge interactions with asymptotic freedom, the running of gauge couplings dictates the running for Yukawa and scalar couplings, and conditions for complete asymptotic freedom have been derived [56] which ensure that gauge theories coupled to matter reach the free UV fixed point [57]. For theories with asymptotic safety, scalars are required to help generate a combined fixed point in the gauge, Yukawa, and quartic scalar couplings. This leads invariably to a "reduction of couplings" and enhanced predictivity over models with asymptotic freedom through a reduced UV critical surface.

\section{Veneziano limit and beyond}

Our findings, throughout, rely on the existence of exact small parameters $\epsilon_{1} \ll 1$ and $\epsilon_{2} \ll 1$ (35) [or $\epsilon \ll 1$ see (41)] in the Veneziano limit, which relate to the gauge one loop coefficients. Consequently, an iterative solution of perturbative beta functions becomes exact and interacting fixed points arise as exact power series in the small parameters. More specifically, the leading non-trivial approximation which is NLO' (Table II) retains the gauge beta functions up to two loop, and the Yukawa and scalar beta functions up to one loop. The parametric smallness of the gauge one-loop coefficients allows an exact cancellation of one and two loop terms implying that interacting fixed points for the gauge couplings must be of the order of the one loop coefficient $\sim \epsilon$. The Yukawa nullclines at one loop imply that Yukawa couplings are necessarily proportional to the gauge couplings, and the scalar nullcline impose that scalar couplings are proportional to the Yukawas (see Sec. VA); hence either of these come out $\sim \epsilon$. Higher order loop approximations $n \mathrm{NLO}^{\prime}$ starting with $n=2$ then correspond to retaining $n+1$ loops in the gauge, and $n$ loops in the Yukawa and scalar beta functions respectively, see Table II. Hence, solving the beta functions for interacting fixed points order-by-order in perturbation theory $(n \rightarrow n+1)$ we have that

$$
\alpha_{i}^{*}=\left.\alpha_{i}^{*}\right|_{n \mathrm{NLO}}+\mathcal{O}\left(\epsilon^{n+1}\right)
$$

for all couplings (26), (27) and all fixed points, with corrections from the $(n+1) \mathrm{NLO}^{\prime}$ level being at least one power in $\epsilon$ smaller than those from the preceding level. We conclude that the expressions for the interacting fixed points $\left.\alpha_{i}^{*}\right|_{n \mathrm{NLO}^{\prime}}$ are accurate polynomials in $\epsilon$ up to including terms of order $\epsilon^{n}$, for all $n$.

Beyond the Veneziano limit, the parametrically small control parameter $\epsilon$ is no longer available. Instead, $\epsilon$ will take finite, possibly large, values dictated by the (finite) field multiplicities. Still, for sufficiently large matter field multiplicities, $\epsilon$ remains sufficiently small and perturbativity remains in reach [38]. It is then conceivable that the fixed points found in the Veneziano limit persist even for finite $N .{ }^{5}$ At finite $N$, however, we stress that the $n N^{\prime} \mathrm{O}^{\prime}$ approximations and (96) are no longer exact order-byorder. It then becomes important to check numerical convergence of higher loop approximations, including nonperturbative resummations. In this context it would be particularly useful to know the radius of convergence of beta functions (in $\epsilon$ ) in the Veneziano limit. A finite radius of convergence has been established rigorously in certain large- $N_{F}$ limits of gauge theories without Yukawa interactions $[58,59]$ which makes it conceivable that the radius of convergence might be finite here as well. ${ }^{6}$ If so, this would offer additional indications for the existence of interacting fixed points beyond the Veneziano limit.

\section{Conformal symmetry and conformal windows}

By their very definition, the gauge-Yukawa theories investigated here are scale-invariant at (interacting) fixed points. Conditions under which scale invariance entails exact conformal invariance have been discussed by Polchinski [60] (see also [61]). Applied to the theories (25) at weak coupling, it implies that exact conformal invariance is realized at all interacting fixed points discovered here. It would then be interesting to find the full conformally invariant effective action beyond the classically marginal invariants retained in (25). First steps into these directions have been reported in [33]. Moreover, for a quantum theory to be compatible with unitarity, scaling dimension of (primary) scalar fields must be larger than unity. This is confirmed for all fixed points by using the results of Sec. III D for the anomalous dimensions of fields and composite scalar operators, together with the results for fixed points at NLO' $^{\prime}$ accuracy (Tables VII and VIII). We conclude that the residual interactions are compatible with unitarity.

Away from the Veneziano limit, findings for the various interacting conformal fixed points persist once $\epsilon$ is finite.

\footnotetext{
${ }^{5}$ An example for a conformal window with asymptotic safety is given in [37] for the model introduced in [32].

${ }^{6}$ Results for resummed beta functions of large- $N$ gauge theories with Yukawa couplings are presently not available.
} 
One may then think of keeping the parameters in the gauge sectors $\left(N_{\mathrm{C}}, N_{\mathrm{c}}\right)$ fixed and finite while varying the matter field content $\left(N_{\mathrm{F}}, N_{\mathrm{f}}, N_{\psi}\right)$. Then, the domain of existence for each of the interacting fixed points (Tables VII, VIII) turns into a "conformal window" as a function of the matter field multiplicities. The fixed point ceases to exist outside the conformal window. The conformal window for asymptotic safety with a simple $S U(N)$ gauge factor has been determined in [37]. Boundaries of conformal windows can be estimated within perturbation theory though more accurate results invariably require nonperturbative tools. ${ }^{7}$

\section{SUMMARY}

We have used perturbation theory and large- $N$ techniques for a rigorous and comprehensive investigation of weakly interacting fixed points of gauge theories coupled to fermionic and scalar matter. For concrete families of simple and semisimple gauge theories with action (25) and following the classification of fixed points put forward in [34,35], we have discovered a large variety of exact highand low-energy fixed points (Tables III, VII, VIII). These include partially interacting ones (Table VII) where one gauge sector remains free, and fully interacting ones (Table VIII) where both gauge sectors are interacting. We have determined the domains of existence for all of them (Figs. 1-5). Interestingly, we also find that the requirement of vacuum stability always singles out a unique viable fixed point in the scalar sector.

As a function of field multiplicities, the phase space of distinct quantum field theories (Fig. 6) includes models with asymptotic safety and asymptotic freedom, and effective theories without UV completion (Figs. 7, 8, and 9). In the IR, theories display either strong coupling and confinement, or weakly coupled fixed points where the elementary gauge fields and fermions are unconfined and appear as massless particles. Many features are a consequence of the semisimple nature and would not arise in simple (or direct products of simple) gauge theories. Highlights include massless semisimple gauge-matter theories where one gauge sector can be both UV free and IR free owing to a fixed point in the other, Fig. 10(c), and theories with inequivalent scaling limits in the IR. Semisimple effects are particularly pronounced for asymptotically free theories where they enhance the diversity of different IR scaling regimes (Fig. 7).

Another central outcome of our study is the first explicit "proof of existence" for asymptotic safety in semisimple quantum field theories with elementary gauge fields, scalars and fermions. It establishes the important result that asymptotic safety is not limited to simple gauge factors [32], fully in line with general theorems and structural results [34]. Our findings, together with their

\footnotetext{
${ }^{7}$ See [62] for lattice studies of conformal windows in QCD with fermionic matter (Banks-Zaks fixed points).
}

supersymmetric counterparts in [36], make it conceivable that semisimple theories display interacting UV fixed points even beyond the Veneziano limit, thus further paving the way for asymptotic safety beyond the Standard Model [38]. The stability of the vacuum (Sec. V) in all models studied here suggests that the near-criticality of the standard model Higgs [39,40] can very well expand into full criticality at an interacting UV fixed point [38].

In addition, we have investigated phase diagrams for simple and semisimple gauge theories with and without Yukawa interactions, continuing an analysis initiated in $[34,35]$. We find that transitions from the UV to the IR can proceed from free or interacting fixed points to confinement and strong coupling. We also find transitions from free to interacting (Figs. 10-15) or from interacting to other interacting conformal fixed points (Fig. 16). In the latter cases, theories display a variety of exact "IR sinks," meaning free or interacting IR conformal fixed points which are fully attractive in all classically marginal interactions. Once more, many new features have come to light beyond those observed in simple gauge theories [34,35].

Our study used minimal models with a low number of Yukawa and gauge couplings. Already at this basic level, an intriguing diversity of fixed points and scaling regimes has emerged, with many novel characteristics both at high and low energies. We believe that these findings warrant more extensive studies in view of rigorous results [34,36], extensions towards strong coupling [33], and its exciting potential for physics beyond the standard model [38].

\section{ACKNOWLEDGMENTS}

Some of the results have been presented at the workshops The Exact Renormalization Group, Trieste (Sept 2016), and Understanding the LHC, Bad Honnef (Feb 2017). This work is supported by the Science and Technology Research Council (STFC) under the Consolidated Grant [ST/ L000504/1] and by an STFC studentship.

\section{APPENDIX A: GENERAL EXPRESSIONS FOR FIXED POINTS}

Most results in the main text relate to the choice $N_{\psi}=1$. For completeness, we summarize fixed point results for general $N_{\psi}$ species of fermions in the fundamental of both gauge groups $S U\left(N_{C}\right)$ and $S U\left(N_{c}\right)$. We observe that $N_{\psi}$ is restricted within the range

$$
0 \leq N_{\psi} \leq \frac{11}{2} .
$$

Outside of this range, exact perturbativity is lost. Substituting $N_{\psi}$ into the RG coefficients and solving for fixed points, we find the following expressions at the partially interacting Banks-Zaks fixed points $\mathrm{FP}_{2}$ and $\mathrm{FP}_{3}$, 


$$
\begin{aligned}
& \mathbf{F P}_{2}: \alpha_{1}=-\frac{4}{75} R \epsilon \\
& \mathbf{F P}_{3}: \alpha_{2}=-\frac{4}{75} \frac{P \epsilon}{R}
\end{aligned}
$$

At the partially interacting fixed points $\mathrm{FP}_{4}$ and $\mathrm{FP}_{5}$ we have

$$
\begin{gathered}
\mathbf{F P}_{4}:\left\{\begin{array}{l}
\alpha_{1}=\frac{2}{3} \frac{13-2 N_{\psi} R}{\left(2 N_{\psi} R-1\right)\left(3 N_{\psi} R-19\right)} R \epsilon \\
\alpha_{Y}=\frac{4}{\left(2 N_{\psi} R-1\right)\left(3 N_{\psi} R-19\right)} R \epsilon
\end{array}\right. \\
\mathbf{F P}_{5}:\left\{\begin{array}{l}
\alpha_{2}=\frac{2}{3} \frac{13-2 N_{\psi} / R}{\left(2 N_{\psi /} / R-1\right)\left(3 N_{\psi} / R-19\right)} \frac{P \epsilon}{R} \\
\alpha_{y}=\frac{4}{\left(2 N_{\psi /} / R-1\right)\left(3 N_{\psi} / R-19\right)} \frac{P \epsilon}{R}
\end{array}\right.
\end{gathered}
$$

For the Banks-Zaks times Banks-Zaks-type fixed point $\mathrm{FP}_{6}$ we find

$$
\mathbf{F P}_{6}:\left\{\begin{array}{l}
\alpha_{1}=-\frac{4}{3}\left(\frac{25-2 N_{\psi} P / R}{625-4 N_{\psi}^{2}}\right) R \epsilon \\
\alpha_{2}=-\frac{4}{3}\left(\frac{25-2 N_{\psi} R / P}{625-4 N_{\psi}^{2}}\right) \frac{P \epsilon}{R}
\end{array}\right.
$$

For the interacting fixed points $\mathrm{FP}_{7}$ and $\mathrm{FP}_{8}$ we find

$$
\begin{aligned}
& \mathbf{F P}_{7}:\left\{\begin{array}{l}
\alpha_{1}=\frac{2}{3}\left(\frac{\left(13-2 N_{\psi} R\right)\left(25-2 N_{\psi} P / R\right)}{150 N_{\psi}^{2} R^{2}-\left(4 N_{\psi}^{\psi}+1025\right) N_{\psi} R+26 N_{\psi}^{2}+475}\right) R \epsilon \\
\alpha_{2}=-\frac{4}{3}\left(\frac{\left(13-2 N_{\psi} R\right) N_{\psi} R / P+\left(2 N_{\psi} R-1\right)\left(3 N_{\psi} R-19\right)}{150 N_{\psi}^{2} R^{2}-\left(4 N_{\psi}^{2}+1025\right) N_{\psi} R+26 N_{\psi}^{2}+475}\right) \frac{P \epsilon}{R} \\
\alpha_{Y}=\frac{4\left(25-2 N_{\psi} P / R\right)}{150 N_{\psi}^{2} R^{2}-\left(4 N_{\psi}^{2}+1025\right) N_{\psi} R+26 N_{\psi}^{2}+475} R \epsilon
\end{array}\right. \\
& \mathbf{F P}_{8}:\left\{\begin{array}{l}
\alpha_{1}=-\frac{4}{3}\left(\frac{\left(13-2 N_{\psi} / R\right) N_{\psi} P / R+\left(2 N_{\psi} / R-1\right)\left(3 N_{\psi} / R-19\right)}{150 N_{\psi}^{2} / R^{2}-\left(4 N_{\psi}^{2}+1025\right) N_{\psi} / R+26 N_{\psi}^{2}+475}\right) R \epsilon \\
\alpha_{2}=\frac{2}{3}\left(\frac{\left(13-2 N_{\psi} / R\right)\left(25-2 N_{\psi} R / P\right)}{150 N_{\psi}^{2} / R^{2}-\left(4 N_{\psi}^{2}+1025\right) N_{\psi} / R+26 N_{\psi}^{2}+475}\right) \frac{P \epsilon}{R} \\
\alpha_{y}=\frac{4\left(25-2 N_{\psi} R / P\right)}{150 N_{\psi /}^{2} / R^{2}-\left(4 N_{\psi}^{2}+1025\right) N_{\psi} / R+26 N_{\psi}^{2}+475} \frac{P \epsilon}{R}
\end{array}\right.
\end{aligned}
$$

Finally, at the fully interacting fixed point $\mathrm{FP}_{9}$ we have

$$
\mathbf{F P}_{9}:\left\{\begin{aligned}
\alpha_{1} & =\frac{2}{3} \frac{\left(13-2 N_{\psi} R\right)\left[\left(13-2 N_{\psi} R\right) N_{\psi} P / R+\left(2 N_{\psi} / R-1\right)\left(3 N_{\psi} / R-19\right)\right] R \epsilon}{114 N_{\psi}^{2}\left(R^{2}+1 / R^{2}\right)+\left(32 N_{\psi}^{4}+1512 N_{\psi}^{2}+361\right)-\left(220 N_{\psi}^{2}+779\right)(R+1 / R)} \\
\alpha_{2} & =\frac{2}{3} \frac{\left(13-2 N_{\psi} / R\right)\left[\left(13-2 N_{\psi} / R\right) N_{\psi} R / P+\left(2 N_{\psi} R-1\right)\left(3 N_{\psi} R-19\right)\right] P \epsilon / R}{114 N_{\psi}^{2}\left(R^{2}+1 / R^{2}\right)+\left(32 N_{\psi}^{4}+1512 N_{\psi}^{2}+361\right)-\left(220 N_{\psi}^{\psi}+779\right)(R+1 / R)} \\
\alpha_{Y} & =\frac{4\left[\left(13-2 N_{\psi} R\right) N_{\psi} P / R+\left(2 N_{\psi} / R-1\right)\left(3 N_{\psi} / R-19\right)\right] R \epsilon}{114 N_{\psi}^{2}\left(R^{2}+1 / R^{2}\right)+\left(32 N_{\psi}^{4}+1512 N_{\psi}^{2}+361\right)-\left(220 N_{\psi}^{2}+779\right)(R+1 / R)} \\
\alpha_{y} & =\frac{4\left[\left(13-2 N_{\psi} / R\right) N_{\psi} R / P+\left(2 N_{\psi} R-1\right)\left(3 N_{\psi} R-19\right)\right] P \epsilon / R}{114 N_{\psi}^{2}\left(R^{2}+1 / R^{2}\right)+\left(32 N_{\psi}^{4}+1512 N_{\psi}^{2}+361\right)-\left(220 N_{\psi}^{2}+779\right)(R+1 / R)} .
\end{aligned}\right.
$$

All expressions reduce to those given in the main body in the limit $N_{\psi}=1$. We note that the parameter range in which fixed points exist changes both qualitatively and quantitatively when varying $N_{\psi}$ within the range (A1). Moreover, we also observe that the characteristic boundaries in paramater space depend on $N_{\psi}$, indicating that domains of existence and eigenvalue spectra depend on $N_{\psi}$. It is straightforward, if tedious, to investigate regions of validity and scaling exponents for the general case, and to find the analogues of Figs. 1-5 and of Tables VI-VIII for general $N_{\psi}$.

\section{APPENDIX B: BOUNDARIES}

We find that the existence and relevancy of fixed points in the parameter space $(P, R)$, see $(61)$, is controlled by characteristic curves $P=X(R), Y(R), \tilde{X}(R)$ or $\tilde{Y}(R)$ with the functions

$$
\begin{array}{ll}
X(R)=\frac{(2 R-13) R}{(2 R-1)(3 R-19)}, & Y(R)=\frac{25}{2} R, \\
\tilde{X}(R)=\frac{(2 / R-1)(3 / R-19)}{(2 / R-13) / R}, & \tilde{Y}(R)=\frac{2}{25} R .
\end{array}
$$

These appear as boundaries of the "phase space" of parameters $(R, P)$ characterizing valid fixed points. Note that the functions $(X, \tilde{X})$ and $(Y, \tilde{Y})$ in (B1) are "dual" to each other,

$$
X(R) \cdot \tilde{X}\left(R^{-1}\right)=1=Y(R) \cdot \tilde{Y}\left(R^{-1}\right) .
$$

A further set of boundaries is given by the straight lines $R=R_{\text {low }}$ or $R_{\text {high }}$, with

$$
R_{\text {low }}=\frac{1}{2} \quad R_{\text {high }}=2 .
$$


The boundaries $P=X(R), Y(R), \tilde{X}(R)$, or $\tilde{Y}(R)$ with (B1) together with (B3) delimit the qualitatively different quantum field theories in the "phase space" shown in Fig. 6.

Certain characteristic values for the parameter $R$ arise in its domain of vailidity $\frac{2}{11}<R<\frac{11}{2}$ at points where the boundaries (B1) cross. We find four of these $R_{1, \ldots, 4}$ with

$$
\frac{2}{11}<R_{\text {low }}<R_{1}<R_{2}<1<R_{3}<R_{4}<R_{\text {high }}<\frac{11}{2},
$$

with $R_{1}$ and $R_{2}$ arising from

$$
\begin{aligned}
& X\left(R_{1}\right)=Y\left(R_{1}\right), \\
& X\left(R_{2}\right)=\tilde{X}\left(R_{2}\right)
\end{aligned}
$$

together with $R_{3}=1 / R_{2}$ and $R_{4}=1 / R_{1}$. Quantitatively we have (62) for $R_{1, \ldots, 4}$ as stated in the main text. The expressions (B1), (B3) for the boundaries are modified once $N_{\psi} \neq 1$.
[1] D. J. Gross and F. Wilczek, Ultraviolet Behavior of Nonabelian Gauge Theories, Phys. Rev. Lett. 30, 1343 (1973).

[2] H. D. Politzer, Reliable Perturbative Results for Strong Interactions?, Phys. Rev. Lett. 30, 1346 (1973).

[3] K. G. Wilson, Renormalization group and critical phenomena. 1. Renormalization group and the Kadanoff scaling picture, Phys. Rev. B 4, 3174 (1971).

[4] S. Weinberg, in General Relativity: An Einstein Centenary Survey, edited by S. W. Hawking and W. Israel (Cambridge University Press, Cambridge, England, 1979), p. 790 .

[5] K. Falls, D. F. Litim, K. Nikolakopoulos, and C. Rahmede, A bootstrap towards asymptotic safety, arXiv:1301.4191.

[6] R. Gastmans, R. Kallosh, and C. Truffin, Quantum gravity near two-dimensions, Nucl. Phys. B133, 417 (1978).

[7] E. Tomboulis, 1/N expansion and renormalization in quantum gravity, Phys. Lett. 70B, 361 (1977).

[8] S. Christensen and M. Duff, Quantum gravity in two $+\epsilon$ dimensions, Phys. Lett. 79B, 213 (1978).

[9] E. Tomboulis, Renormalizability and asymptotic freedom in quantum gravity, Phys. Lett. 97B, 77 (1980).

[10] M. E. Peskin, Critical point behaviour of the Wilson loop, Phys. Lett. 94B, 161 (1980).

[11] L. Smolin, A fixed point for quantum gravity, Nucl. Phys. B208, 439 (1982).

[12] W. A. Bardeen, M. Moshe, and M. Bander, Spontaneous Breaking of Scale Invariance and the Ultraviolet Fixed Point in $\mathrm{O}(n)$ Symmetric $\phi_{3 d}^{6}$ Theory, Phys. Rev. Lett. 52, 1188 (1984).

[13] K. Gawedzki and A. Kupiainen, Exact Renormalization for the Gross-Neveu Model of Quantum Fields, Phys. Rev. Lett. 54, 2191 (1985).

[14] K. Gawedzki and A. Kupiainen, Renormalizing the Nonrenormalizable, Phys. Rev. Lett. 55, 363 (1985).

[15] C. de Calan, P. Faria da Veiga, J. Magnen, and R. Seneor, Constructing the Three-Dimensional Gross-Neveu Model with a Large Number of Flavor Components, Phys. Rev. Lett. 66, 3233 (1991).

[16] D. Kazakov and G. Vartanov, Renormalizable $1 / \mathrm{N}(\mathrm{f})$ expansion for field theories in extra dimensions, J. High Energy Phys. 06 (2007) 081.
[17] M. Reuter, Nonperturbative evolution equation for quantum gravity, Phys. Rev. D 57, 971 (1998).

[18] D. F. Litim, Fixed Points of Quantum Gravity, Phys. Rev. Lett. 92, 201301 (2004).

[19] D. F. Litim, On fixed points of quantum gravity, AIP Conf. Proc. 841, 322 (2006).

[20] M. Niedermaier, The asymptotic safety scenario in quantum gravity: An Introduction, Classical Quantum Gravity 24, R171 (2007).

[21] D. F. Litim, Fixed points of quantum gravity and the renormalisation group, Proc. Sci., QG-Ph (2007) 024.

[22] A. Codello, R. Percacci, and C. Rahmede, Investigating the ultraviolet properties of gravity with a Wilsonian renormalization group equation, Ann. Phys. (Amsterdam) 324, 414 (2009).

[23] D. F. Litim, Renormalisation group and the Planck scale, Phil. Trans. R. Soc. A 369, 2759 (2011).

[24] K. Falls, D. F. Litim, K. Nikolakopoulos, and C. Rahmede, Further evidence for asymptotic safety of quantum gravity, Phys. Rev. D 93, 104022 (2016).

[25] J. Biemans, A. Platania, and F. Saueressig, Quantum gravity on foliated spacetimes: Asymptotically safe and sound, Phys. Rev. D 95, 086013 (2017).

[26] S. Folkerts, D. F. Litim, and J. M. Pawlowski, Asymptotic freedom of Yang-Mills theory with gravity, Phys. Lett. B 709, 234 (2012).

[27] N. Christiansen, D. F. Litim, J. M. Pawlowski, and A. Rodigast, Fixed points and infrared completion of quantum gravity, Phys. Lett. B 728, 114 (2014).

[28] P. Don, A. Eichhorn, and R. Percacci, Matter matters in asymptotically safe quantum gravity, Phys. Rev. D 89, 084035 (2014).

[29] J. Meibohm, J. M. Pawlowski, and M. Reichert, Asymptotic safety of gravity-matter systems, Phys. Rev. D 93, 084035 (2016).

[30] N. Christiansen, D. F. Litim, J. M. Pawlowski, and M. Reichert, One force to rule them all: asymptotic safety of gravity with matter, arXiv:1710.04669.

[31] K. Falls, C. R. King, D. F. Litim, K. Nikolakopoulos, and C. Rahmede, Asymptotic safety of quantum gravity beyond Ricci scalars, arXiv:1801.00162.

[32] D. F. Litim and F. Sannino, Asymptotic safety guaranteed, J. High Energy Phys. 12 (2014) 178. 
[33] T. Buyukbese and D. F. Litim, Asymptotic safety of gauge theories beyond marginal interactions, Proc. Sci., LATTICE2016 (2017) 233.

[34] A. D. Bond and D. F. Litim, Theorems for asymptotic safety of gauge theories, Eur. Phys. J. C 77, 429 (2017).

[35] A. Bond and D. Litim, Interacting ultraviolet completions of four-dimensional gauge theories, Proc. Sci., LATTICE2016 (2017) 208.

[36] A. D. Bond and D. F. Litim, Asymptotic Safety Guaranteed in Supersymmetry, Phys. Rev. Lett. 119, 211601 (2017).

[37] A. D. Bond, D. F. Litim, G. Medina Vazquez, and T. Steudtner, UV conformal window for asymptotic safety, Phys. Rev. D 97, 036019 (2018).

[38] A. D. Bond, G. Hiller, K. Kowalska, and D. F. Litim, Directions for model building from asymptotic safety, J. High Energy Phys. 08 (2017) 004.

[39] J. Elias-Miro, J. R. Espinosa, G. F. Giudice, G. Isidori, A. Riotto, and A. Strumia, Higgs mass implications on the stability of the electroweak vacuum, Phys. Lett. B 709, 222 (2012).

[40] D. Buttazzo, G. Degrassi, P. P. Giardino, G. F. Giudice, F. Sala, A. Salvio, and A. Strumia, Investigating the near-criticality of the Higgs boson, J. High Energy Phys. 12 (2013) 089.

[41] M. E. Machacek and M. T. Vaughn, Two loop renormalization group equations in a general quantum field theory. 1 . Wave function renormalization, Nucl. Phys. B222, 83 (1983).

[42] M. E. Machacek and M. T. Vaughn, Two loop renormalization group equations in a general quantum field theory. 2 . Yukawa couplings, Nucl. Phys. B236, 221 (1984).

[43] M. E. Machacek and M. T. Vaughn, Two loop renormalization group equations in a general quantum field theory. 3 . Scalar quartic couplings, Nucl. Phys. B249, 70 (1985).

[44] M.-x. Luo, H.-w. Wang, and Y. Xiao, Two loop renormalization group equations in general gauge field theories, Phys. Rev. D 67, 065019 (2003).

[45] F. Dyson, Divergence of perturbation theory in quantum electrodynamics, Phys. Rev. 85, 631 (1952).

[46] W. E. Caswell, Asymptotic Behavior of Nonabelian Gauge Theories to Two Loop Order, Phys. Rev. Lett. 33, 244 (1974).

[47] T. Banks and A. Zaks, On the phase structure of vector-like gauge theories with massless fermions, Nucl. Phys. B196, 189 (1982).
[48] D. F. Litim, M. Mojaza, and F. Sannino, Vacuum stability of asymptotically safe gauge-Yukawa theories, J. High Energy Phys. 01 (2016) 081.

[49] G. Veneziano, U(1) without instantons, Nucl. Phys. B159, 213 (1979).

[50] G. 't Hooft, Naturalness, chiral symmetry, and spontaneous chiral symmetry breaking, edited by G. 't Hooft et al., Recent Developments in Gauge Theories. NATO Advanced Study Institutes Series, vol. 59 (Springer, Boston, MA, 1980), pp. 135-157.

[51] A. Paterson, Coleman-Weinberg symmetry breaking in the chiral $\mathrm{SU}(\mathrm{N}) \times \mathrm{SU}(\mathrm{N})$ linear sigma model, Nucl. Phys. B190, 188 (1981).

[52] D. J. E. Callaway, Triviality pursuit: Can elementary scalar particles exist?, Phys. Rep. 167, 241 (1988).

[53] K. Wilson and J. B. Kogut, The renormalization group and the epsilon expansion, Phys. Rep. 12, 75 (1974).

[54] M. Luscher and P. Weisz, Scaling laws and triviality bounds in the lattice $\phi^{4}$ theory. 2 . One component model in the phase with spontaneous symmetry breaking, Nucl. Phys. B295, 65 (1988).

[55] A. Hasenfratz, K. Jansen, C. B. Lang, T. Neuhaus, and H. Yoneyama, The triviality bound of the four component $\phi^{4}$ model, Phys. Lett. B 199, 531 (1987).

[56] S. R. Coleman and D. J. Gross, Price of Asymptotic Freedom, Phys. Rev. Lett. 31, 851 (1973).

[57] N.-P. Chang, Eigenvalue conditions and asymptotic freedom for Higgs scalar gauge theories, Phys. Rev. D 10, 2706 (1974).

[58] A. Palanques-Mestre and P. Pascual, The $1 / N_{f}$ expansion of the $\gamma$ and beta functions in QED, Commun. Math. Phys. 95, 277 (1984).

[59] B. Holdom, Large N flavor beta-functions: A recap, Phys. Lett. B 694, 74 (2010).

[60] J. Polchinski, Scale and conformal invariance in quantum field theory, Nucl. Phys. B303, 226 (1988).

[61] M. A. Luty, J. Polchinski, and R. Rattazzi, The $a$-theorem and the asymptotics of 4D quantum field theory, J. High Energy Phys. 01 (2013) 152.

[62] L. Del Debbio, The conformal window on the lattice, Proc. Sci., Lattice2010 (2014) 004, [arXiv:1102.4066]. 